ACCepted for publication in the Astrophysical Journal

Preprint typeset using $\mathrm{LAT}_{\mathrm{E} X} \mathrm{X}$ style emulateapj v. 5/2/11

\title{
A SPECTROSCOPIC SURVEY AND ANALYSIS OF BRIGHT, HYDROGEN-RICH WHITE DWARFS
}

\author{
A. Gianninas ${ }^{1,3}$, P. Bergeron ${ }^{1}$, and M. T. Ruiz ${ }^{2,3}$ \\ ${ }^{1}$ Département de Physique, Université de Montréal, C.P. 6128, Succ. Centre-Ville, Montréal, Québec H3C 3J7, Canada; \\ ${ }^{2}$ Departamento de Astronomía, Universidad de Chile, Casilla 36-D, Santiago, Chile and \\ ${ }^{3}$ Visiting astronomers at Las Campanas Observatory operated by Carnegie Institution of Washington \\ Accepted for publication in the Astrophysical Journal
}

\section{ABSTRACT}

We have conducted a spectroscopic survey of over 1300 bright $(V \leq 17.5)$, hydrogen-rich white dwarfs based largely on the last published version of the McCook \& Sion catalog. The complete results from our survey, including the spectroscopic analysis of over 1100 DA white dwarfs, are presented. High signal-to-noise ratio optical spectra were obtained for each star and were subsequently analyzed using our standard spectroscopic technique where the observed Balmer line profiles are compared to synthetic spectra computed from the latest generation of model atmospheres appropriate for these stars. First, we present the spectroscopic content of our sample, which includes many misclassifications as well as several DAB, DAZ, and magnetic white dwarfs. Next, we look at how the new Stark broadening profiles affect the determination of the atmospheric parameters. When necessary, specific models and analysis techniques are used to derive the most accurate atmospheric parameters possible. In particular, we employ $\mathrm{M}$ dwarf templates to obtain better estimates of the atmospheric parameters for those white dwarfs that are in DA+dM binary systems. Certain unique white dwarfs and doubledegenerate binary systems are also analyzed in greater detail. We then examine the global properties of our sample including the mass distribution and their distribution as a function of temperature. We then proceed to test the accuracy and robustness of our method by comparing our results to those of other surveys such as SPY and Sloan Digital Sky Survey. Finally, we revisit the ZZ Ceti instability strip and examine how the determination of its empirical boundaries is affected by the latest line profile calculations.

Subject headings: binaries: spectroscopic - stars: fundamental parameters - surveys - techniques: spectroscopic - white dwarfs

\section{INTRODUCTION}

For many years, the catalog of McCook \& Sion (1999, hereafter MS99) was the most important reference in the white dwarf community including a total of 2249 spectroscopically identified white dwarf stars. Similarly, the now regularly updated online incarnation 1 is used by white dwarf researchers on a daily basis and has grown to include nearly 13,000 entries as this publication goes to press. This is due in large part to the increasingly large number of white dwarfs discovered in the Sloan Digital Sky Survey (SDSS). The SDSS's huge database of newly discovered white dwarfs has permitted the discovery of previously unknown types of white dwarfs like the carbon atmosphere "Hot DQ" stars (Dufour et al. 2008), white dwarfs with gaseous disks (Gänsicke et al. 2008), and even white dwarfs with oxygen dominated atmospheres (Gänsicke et al. 2010). However, the fact remains that most of the SDSS data lack the necessary signal-to-noise ratio $(\mathrm{S} / \mathrm{N})$ to measure accurately the atmospheric parameters for each star (Gianninas et al. 2005). This is largely due to the rather faint white dwarfs observed by the SDSS and the practice of having a set exposure time for every field. Consequently, the fainter the star, the lower the $\mathrm{S} / \mathrm{N}$ of the data. On the other hand, white dwarfs from MS99 are comparatively bright, especially if we restrict ourselves to stars with $V \leq 17.5$. Historically, many of the white dwarfs from MS99 have not been particularly well studied despite their relative brightness, which makes it easier to obtain high $\mathrm{S} / \mathrm{N}$ spectroscopic observations. Indeed, many of these white dwarfs have only a spectral classification, based on photographic spectra, without any further analysis ever having been performed, let alone with modern CCD spectroscopy. Therefore, we believe that it is both important and worthwhile to study these stars, the more so that it is much more feasible to conduct follow-up observations (i.e., high-resolution spectroscopy, high-speed photometry, etc.) for objects of interest, if they are bright.

Indeed, some of the early results from our survey have already allowed us to make a few discoveries of our own. The most notable of these is likely the discovery of the unique nature of GD 362, originally reported as a highly metallic and massive DAZ white dwarf (Gianninas et al. 2004), it was later discovered that the main atmospheric constituent is helium (Zuckerman et al. 2007). Even more important was the discovery of an infrared excess due to the presence of a circumstellar debris disk (Becklin et al. 2005; Kilic et al. 2005) around this star. This lone discovery essentially opened the door to a whole new area of white dwarf research with many groups launching observational campaigns with the specific goal of discovering debris disks around other cool, metalenriched white dwarfs. We have also successfully used our survey to refine the empirical determination of the boundaries of the ZZ Ceti instability strip and con@dimncitise.plurity (Gianninas et al. 2005). We were thus

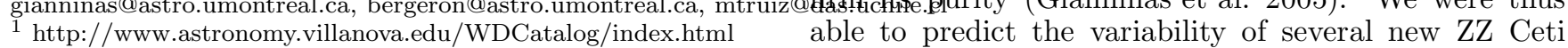


pulsators, while also confirming the non-variability of other DA white dwarfs near the ZZ Ceti instability strip (Gianninas et al. 2006).

Ours is not the only survey to have recently looked at a substantial number of bright white dwarfs. The ESO SN Ia Progenitor Survey (SPY; Koester et al. 2001) also observed about 800 bright white dwarfs, of which many were selected from MS99. It is not surprising then that there is a rather large overlap between the SPY sample and our own. However, in the case of SPY, the aim of the survey was to obtain multi-epoch, high-resolution spectra in order to detect the radial velocity variations of double-degenerate binary systems. These could then lead to the identification of possible SN Ia progenitors, which were ultimately what they were looking to find. Results of the analysis of the DA white dwarfs from SPY are summarized in Koester et al. (2009). Finlev et al. (1997) also presented an analysis of 174 hot $\left(T_{\text {eff }}>25,000 \mathrm{~K}\right)$ DA white dwarfs selected from MS99 as well as the ROSAT (Pounds et al. 1993) and Extreme-Ultraviolet Explorer (EUVE; Bowyer et al. 1994) surveys. Similarly, Marsh et al. (1997a,b) and Vennes et al. (1996, 1997, 1998) also presented analyses of DA white dwarfs selected from ROSAT and EUVE, while Vennes et al. (2002) analyzed over $200 \mathrm{DA}$ white dwarfs discovered in the $2 \mathrm{dF}$ QSO Redshift Survey (Smith et al. 2005). Other important studies of large samples of DA white dwarfs include the works of Liebert et al. (2005, hereafter LBH05) and Limoges \& Bergeron (2010) who looked at the complete samples from the Palomar-Green (PG; Green et al. 1986) and Kiso (KUV; Noguchi et al. 1980; Kondo et al. 1984) surveys, respectively. These studies defined statistically significant samples of white dwarfs in an effort to derive the luminosity function of the disk white dwarf population. Finally, an analysis of over 7000 DA white dwarfs from the SDSS Data Release 4 (DR4) was presented in Kepler et al. (2007) with particular attention to the mass distribution of the sample. Tremblav et al. (2011a) performed a new analysis of the aforementioned sample of DA white dwarfs from the SDSS and showed that for the analysis of ensemble properties, such as the mass distribution, the SDSS data are reliable down to $\mathrm{S} / \mathrm{N} \sim 15$. However, the precise determination of the atmospheric parameters for individual stars remains problematic with such low $\mathrm{S} / \mathrm{N}$ observations, as in the case of the ZZ Ceti instability strip (Gianninas et al. 2005).

In an effort to derive the most accurate atmospheric parameters possible, we also want to incorporate all available improvements to the model atmospheres that we use to study these white dwarfs. With that in mind, we will be using models that include the improved theoretical calculations of the Balmer line profiles, including non-ideal effects, by Tremblay \& Bergeron (2009, hereafter TB09). Seeing as our spectroscopic technique relies on a detailed comparison between observed and theoretical Balmer lines, it is crucial that we employ the most accurate and up-to-date models at our disposal. In the same vein, two sub-categories of white dwarfs contained within our sample required particular attention in order to obtain accurate atmospheric parameters. First, there are the hot DA and DAO stars exhibiting the socalled Balmer line problem. A comprehensive and detailed analysis of these stars has already been presented by Gianninas et al. (2010) and those results will be in- cluded in our analysis. Second, we have DA+dM binary systems whose Balmer line profiles are contaminated, to varying degrees, by the spectrum of the $\mathrm{dM}$ secondary.

As a whole, we wish to present a detailed analysis of all the hydrogen line white dwarfs from MS99 for which we have obtained optical spectra. In Section 2, we will present the spectroscopic content of our survey. Section 3 will deal with the model atmospheres used for the analysis of our data, and we will present in Section 4 the spectroscopic analysis of our entire sample, including particular analyses for certain subsets of white dwarfs. Subsequently, in Section 5 we will examine the global properties of our sample including the mass distribution and the mass distribution as a function of effective temperature, and how these compare to other large surveys. We will then revisit the PG luminosity function, and the empirical determination of the ZZ Ceti instability strip. Finally, we end with some concluding remarks in Section 6.

\section{SPECTROSCOPIC CONTENT}

The goal of our survey was to observe all the white dwarfs listed as DA in MS99, including all subtypes $(\mathrm{DAB}, \mathrm{DAH} / \mathrm{P}, \mathrm{DAO}, \mathrm{DAZ}, \mathrm{DA}+\mathrm{dM})$, with a visual magnitude $V \leq 17.5$. Along the way, several DA stars were added to the sample through various other projects and collaborations the Montréal group was involved in. In the final reckoning, we had defined a sample of 1503 white dwarfs for which we wanted to obtain high $\mathrm{S} / \mathrm{N}$ optical spectra. After nearly 8 years of observing, and a total of 19 observing runs in both the northern and southern hemispheres, we succeeded in securing spectra for 1360 of the 1503 white dwarfs we had selected. This translates to a $90 \%$ completion rate. Indeed, the majority of the missing spectra are those of the least bright stars in our sample and objects observable only from the southern hemisphere where we were unable to secure additional telescope time.

In all, 726 spectra were obtained specifically for the purposes of this survey since it began in 2003. Of those 726 spectra, 568 were obtained between 2003 September and 2011 April at Steward Observatory's $2.3 \mathrm{~m}$ telescope equipped with the Boller \& Chivens spectrograph. The 4!. 5 slit together with the 600 line $\mathrm{mm}^{-1}$ grating blazed at $3568 \AA$ in first order provide a spectral coverage from about 3000 to $5250 \AA$ at a resolution of $\sim 6 \AA$ (FWHM). An additional 18 spectra were obtained in 2006 April and 2007 January at the $1.6 \mathrm{~m}$ telescope of the Observatoire du Mont-Mégantic where the 600 line $\mathrm{mm}^{-1}$ grating provided a spectral coverage from about 3100 to $7500 \AA$ at a similar resolution. Furthermore, a total of 140 spectra from the southern hemisphere were secured during the course of two observing runs in 2007 March and 2007 October, respectively. First, 84 spectra were obtained at ESO's $3.6 \mathrm{~m}$ telescope at La Silla, Chile with the ESO Faint Object Spectrograph and Camera (v.2). The \#7 grism and a 1".0 slit provided a spectral coverage from about 3300 to $5200 \AA$ with a resolution of $\sim 6 \AA$ (FWHM). The remaining 56 southern spectra were obtained at Carnegie Observatories' $2.5 \mathrm{~m}$ Irénée du Pont Telescope at Las Campanas, Chile with the Boller \& Chivens spectrograph. The 1".5 slit with the 600 line $\mathrm{mm}^{-1}$ grating blazed at $5000 \AA$ provided a 


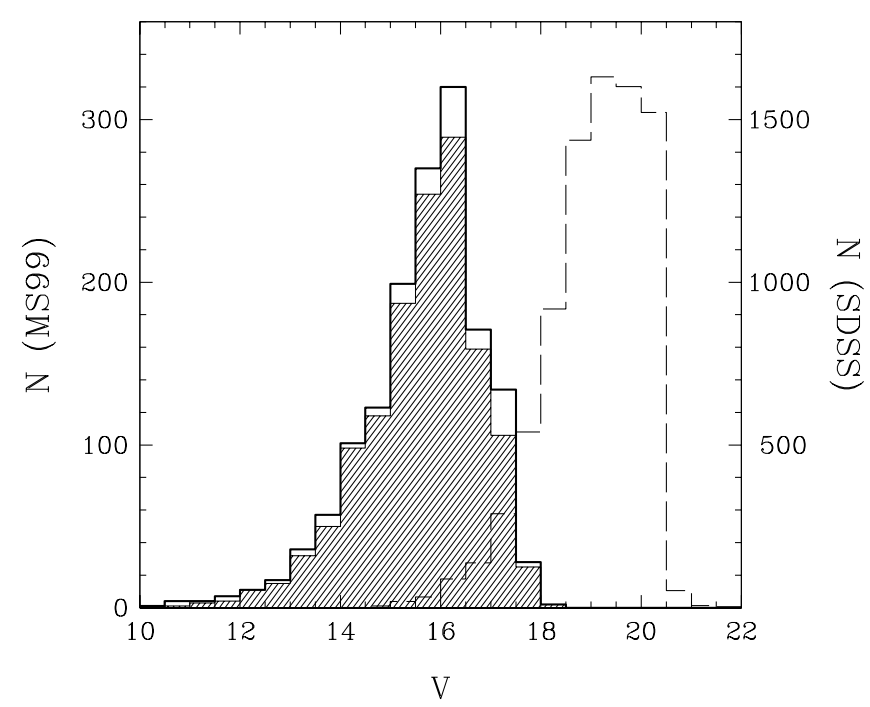

Figure 1. Distribution of visual magnitudes $V$ for our sample selected from MS99 (bold histogram) and for the white dwarfs for which we have obtained optical spectra (hatched histogram). In comparison, the distribution for the SDSS sample as of DR4 (Eisenstein et al. 2006) is also shown (dashed histogram). Note that the scale is different for the McCook \& Sion sample (left) and the SDSS sample (right) and that we use the SDSS $g$ magnitudes as equivalent to $V$.

spectral coverage from about 3500 to $6600 \AA$ at a slightly better resolution of $\sim 3 \AA$ (FWHM). There are also 169 spectra that were kindly provided by C. Moran (1999, private communication). Of these, eight were obtained at the South African Astronomical Observatory's $1.9 \mathrm{~m}$ Radcliffe Telescope using the Grating Spectrograph. The remaining 161 spectra were obtained at the Isaac Newton Telescope, on La Palma, with the Intermediate Dispersion Spectrograph. In both cases, the spectra have a resolution of $\sim 3 \AA$ (FWHM). Finally, the sources of the spectra for $0950+139$ and $1136+667$ (two DAO white dwarfs) are described in Gianninas et al. (2010). For the remaining 464 stars, we have used archival spectra that have been obtained throughout the years by the Montréal group during the course of various other projects (e.g., Bergeron et al. 1992; LBH05). These were acquired at Steward Observatory's $2.3 \mathrm{~m}$ telescope with the same instrument and setup described above.

We show in Figure 1 the final distribution of visual magnitudes $V$ for our sample of white dwarfs (see Figure 1 of Gianninas et al. 2009, as a comparison). We note that 10 white dwarfs have no magnitude listed in the literature. The bold histogram shows the distribution for the entire sample while the hatched histogram represents the stars for which we have successfully obtained spectra. We see that the distribution peaks near $V=16.5$ and that the majority of our stars have $V \leq 16.5$. We contrast this result with the sample of DA white dwarfs from the SDSS DR4 (Eisenstein et al. 2006). Although the SDSS sample contains over six times more white dwarfs, the vast majority of these have $20.5 \geq g \geq 18.5$, which makes for a much fainter sample overall. The distribution of $\mathrm{S} / \mathrm{N}$ for our entire sample of 1360 optical spectra is displayed in Figure2. The $\mathrm{S} / \mathrm{N}^{2}$ is determined by an esti-

${ }^{2}$ For the majority of our spectra, obtained at Steward Observa-

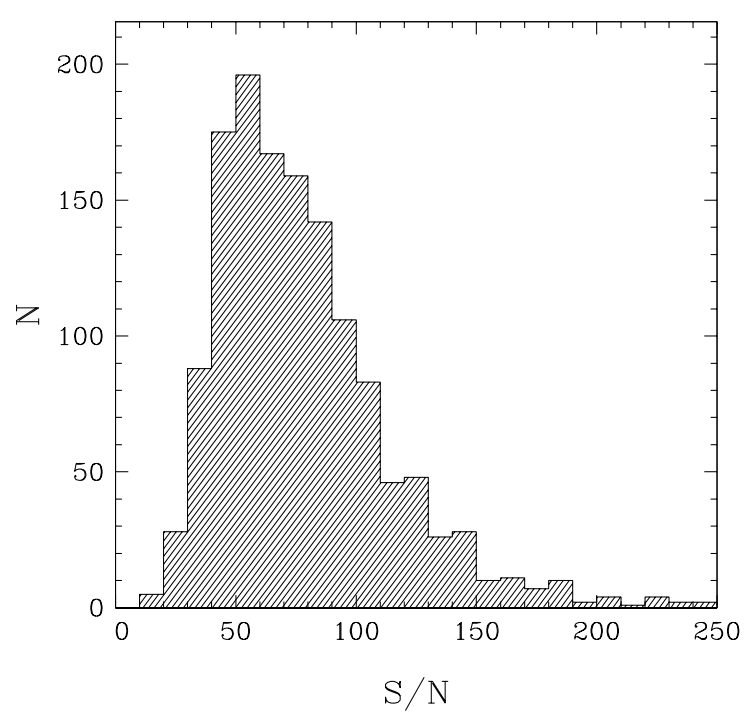

Figure 2. Distribution of $\mathrm{S} / \mathrm{N}$ for all the stars in our spectroscopic survey. The distribution peaks at the 50-60 bin and more than 50\% of the spectra have $\mathrm{S} / \mathrm{N} \geq 70$.

mate of the rms noise per pixel in the continuum between $\sim 4500$ and $\sim 4700 \AA$. Over $52 \%$ of our sample have $\mathrm{S} / \mathrm{N}$ $>70$ and almost $80 \%$ above 50 . Only the faintest objects in our sample have $\mathrm{S} / \mathrm{N}$ that is significantly lower. As such, we have achieved our goal of obtaining high-quality data for the majority of the white dwarfs in our survey.

As previously stated, many of these stars have never been studied using modern CCD spectroscopy. Consequently, it is not surprising to discover that many of the spectral classifications are erroneous; many of the objects listed in MS99 are not even white dwarfs. We list in Table 168 objects that are incorrectly classified as white dwarfs in MS99. We provide the spectral classification from MS99 as well as our revised spectroscopic classification for each object along with the appropriate references. Some of these have been known to be misclassified for some time but are still listed as white dwarfs in the online version of MS99. Of the 68 stars listed in Table 1, 20 were recently reported as being misclassified objects in Limoges \& Bergeron (2010) and we refer the reader to Figure 1 of Limoges \& Bergeron for the spectra of those stars. In this paper, we report the erroneous classification of an additional 27 objects for the first time. The spectra of these 27 white dwarfs are displayed in Figure 3. Many of these are sdB stars that show strong Balmer lines that are not as broad as those of their white dwarf cousins due to their lower surface gravity. However, it is not too hard to see how these objects could easily have been mistaken as white dwarfs, especially using photographic spectra. On the other hand, it is harder to explain how several main-sequence stars were just as easily misclassified. Indeed, even a quasar $(0713+745)$ has found its way into MS99. Furthermore, MS99 still lists stars like BD+28 $4211(2148+286)$ and Feige $34(1036+433)$, popular spectrophotometric standards that are known hot subdwarfs. In addition to the misclassified objects, several stars had two separate en-

tory's $2.3 \mathrm{~m}$ telescope, there are $\sim 3.2$ pixels per resolution element but this value varies for the other instrument setups employed. 
tries in MS99 under two different names. Although virtually all of these have since been corrected, we report here that at least two still remain. The entries for $0525+271$ and $0526+271$ refer to the same star, which also happens to be one of the misclassified objects in Table 1 and $1100+604$ and $1104+602$ are also one in the same. We also note that $1959+059$ is GD 226 , not GD 266 as listed in MS99 (Giclas et al. 1965).

In Figure 4, we show a series of representative DA spectra in order of decreasing effective temperature, all of which have $75 \geq \mathrm{S} / \mathrm{N} \geq 65$. We have chosen this range of $\mathrm{S} / \mathrm{N}$ values to emphasize the mean quality of our data, which is quite high overall. We refer the reader to Gianninas et al. (2010, see Figure 1) for a detailed presentation of the DAO white dwarfs contained within our sample. Spectra for the 25 magnetic white dwarfs in our sample are displayed in Figure 5 as a function of their continuum slope. In many of these we see the classical Zeeman splitting of the hydrogen lines for somewhat lower magnetic field strengths on the order of $\sim 1$ to 10 MG. On the other hand, stars with much stronger fields, on the order of tens to hundreds of MG, are rendered almost unrecognizable as DA stars. Of note in Figure 5 are possibly four new magnetic white dwarfs. With regards to $1018-103$ and $1610+330$, there is little doubt as to their magnetic nature. On the other hand, the cases of 0321-026 and 0350+098 are more problematic. In the case of 0321-026, the Balmer lines predicted by our best-fit model are too deep and we obtain a somewhat high-log $g$ value (see Section 4.6). This is often the case with magnetic white dwarfs as the Zeeman splitting broadens the observed line profiles leading to a higher $\log g$ value. We suggest that $0321-026$ might harbor a weak magnetic field, and a spectrum with higher $\mathrm{S} / \mathrm{N}$ could reveal weak Zeeman splitting such as that seen in $0637+477$. Second, $0350+098$ was detected by ROSAT (Fleming et al. 1996) and we therefore conclude that it must be a relatively hot star, and the slope would seem to corroborate that conclusion. Unfortunately, nothing else is known about this object. The absence of any spectral features could be an indication of a strong magnetic field that has completely diluted any absorption lines that would otherwise be present in the spectrum of $0350+098$. Spectropolarimetric observations would be needed to confirm the magnetic nature of this star. We also note that three of the magnetic white dwarfs are members of double-degenerate binary systems. Namely, 0945+245 (LB 11146, Liebert et al. 1993), 1440+753 (RE J1440+750, Vennes et al. 1999), and 1506+229 (CBS 229, Gianninas et al.|2009).

Finally, in Figure [6 we display spectra for white dwarfs of other miscellaneous spectral types included in our sample. This includes five DAB stars, six DAZ white dwarfs, and eight cool DA. Among the DAB white dwarfs we find HS $0209+0832(0209+085)$ and GD $323(1302+597)$, which have been shown to be spectroscopically variable (Heber et al. 1997; Pereira et al. 2005, respectively), while the other three DAB stars $(0339+002, \quad 0453-295$, and $1115+166)$ are actually DA+DB binary systems (Limoges \& Bergeron 2010; Wesemael et al. 1994; Bergeron \& Liebert 2002, respectively). Regarding the DAZ white dwarfs, our sample includes the first DAZ ever discovered, G74-7 $(0208+396$, Lacombe et al. 1983) as well as GD $362(1729+371$,
Gianninas et al. 2004). Although, as previously mentioned, GD 362 has since been shown to have a heliumdominated atmosphere (Zuckerman et al. 2007), a discovery made possible by the detection of a very faint helium line at $5877 \AA$. However, the dominant absorption lines in the spectrum, which define the spectral class of an object, remain the hydrogen and metal lines, as such the spectral type should at most be updated to DAZB. In addition, we report for the first time that $0543+436$ and $1054-226$ are also members of the DAZ class. In particular, 1054-226 was initially discovered by Subasavage et al. (2007) but their discovery spectrum lacked the necessary $\mathrm{S} / \mathrm{N}$ to detect the $\mathrm{H}$ and $\mathrm{K}$ lines of calcium. In addition, there are eight white dwarfs whose spectra are completely devoid of absorption lines in the observed wavelength range. However, with the exception of $0648+368$ and $1055-072$, the other six cool DA stars have detections of $\mathrm{H} \alpha$ (Bergeron et al. 2001; Silvestri et al. 2001), hence their DA spectral type. We also note that $0727+482$ is actually a double-degenerate system (Strand et al. 1976). Finally, we also include the peculiar spectrum obtained for $1015+076$. It seems that $1015+076$ is a DA white dwarf whose spectrum is completely dominated by an unresolved, background G dwarf (Farihi et al. 2005). Indeed, the SPY spectrum analyzed in Voss (2006) shows a perfectly normal DA white dwarf with $T_{\text {eff }} \sim 25,600 \mathrm{~K}$ and $\log g \sim 7.70$.

Our spectroscopic sample also contains a total of 47 $\mathrm{DA}+\mathrm{dM}$ binary systems, all of which have been previously identified. In order to obtain accurate atmospheric parameters for the DA white dwarfs in these binary systems, we need to remove the contamination from the dM secondary. With that in mind, we have obtained follow-up spectra for all the DA+dM systems in our sample, with the exception of five (0208-153, 0309-275, 0419-487, 1541-381, 1717-345), covering a larger spectral range from 3500 to $7000 \AA$. These spectra were obtained at Steward Observatory's $2.3 \mathrm{~m}$ telescope using the same instrument and setup as described above except that here we used the 400 line $\mathrm{mm}^{-1}$ grating blazed at $4800 \AA$ to achieve the desired wavelength coverage. The one drawback was that the use of this particular grating degrades our spectral resolution to $\sim 9 \AA$ (FWHM) from our usual $\sim 6 \AA$. However, this slightly reduced spectral resolution is still sufficient for our purposes. We present in Figure 7 what we will refer to as our red spectra for 42 of the $47 \mathrm{DA}+\mathrm{dM}$ binaries in our sample. These will be coupled with our usual optical spectra $(3000-5250 \AA)$, which we will call our blue spectra, for the analysis of the $\mathrm{DA}+\mathrm{dM}$ systems. Several of the tell tale features found in the spectra of DA+dM binaries can be seen in Figure 7. We see in these spectra how the contribution from the $\mathrm{M}$ dwarf becomes more significant at longer wavelengths. More specifically, we see the $\mathrm{TiO}$ absorption band near $\sim 4950 \AA$ that produces the "kink" in the red wing of $\mathrm{H} \beta$. We also note the presence of the strong $\mathrm{Na}$ D line near $5895 \AA$ as well as emission, from the M dwarf, in the core of several Balmer lines, especially at $\mathrm{H} \alpha$.

As a summary, we present in Table 2 a breakdown of the white dwarfs in our sample listing the number of stars in each spectral class. 
Table 1

Misclassified Objects

\begin{tabular}{|c|c|c|c|c|}
\hline WD & Name & ST (MS99) & ST (This Work) & Notes \\
\hline $0021-234$ & Ton S 155 & DAWK & $\operatorname{sdB}$ & 1 \\
\hline $0031-274$ & Ton S 163 & DA1 & $\operatorname{sdB}$ & 1 \\
\hline $0107-342$ & GD 687 & DA3 & $\operatorname{sdB}$ & 1 \\
\hline $0109-264$ & Ton S 201 & DA1 & $\operatorname{sdB}$ & 1 \\
\hline $0113-243$ & GD 693 & DA6 & $\operatorname{sdB}$ & 2 \\
\hline $0154-071$ & PB 8949 & DA1 & $\operatorname{sdB}$ & 1 \\
\hline $0200-171$ & G272-B5B & DA & $\operatorname{sdB}$ & 3 \\
\hline $0222+314$ & KUV $02222+3124$ & DA & $\mathrm{sdB}$ & 2 \\
\hline $0240+341$ & KUV $02409+3407$ & DA & $\mathrm{MS}(\mathrm{dF})$ & 2 \\
\hline $0258+184$ & PG $0258+184$ & DA & $\mathrm{sdB}$ & 1 \\
\hline $0340-243$ & Ton S 372 & DAWK & $\mathrm{MS}(\mathrm{dF})$ & 3 \\
\hline $0341-248$ & Ton S 374 & DA & $\mathrm{sdB}$ & 3 \\
\hline $0459+280$ & CTI $045934.1+280335$ & DA & $\mathrm{MS}(\mathrm{dF})$ & 3 \\
\hline $0509+168$ & KUV $05097+1649$ & DA & $\mathrm{MS}(\mathrm{dF})$ & 4 \\
\hline $0510+163$ & KUV 05101+1619 & DA & $\mathrm{MS}(\mathrm{dF})$ & 2 \\
\hline $0526+271$ & KUV $05260+2711$ & DA & $\mathrm{sdB}$ & 2 \\
\hline $0529+261$ & KUV $05296+2610$ & DA & $\operatorname{sdB}$ & 2 \\
\hline $0627+299$ & KUV $06274+2958$ & DA & $\mathrm{sdB}$ & 2 \\
\hline $0628+314$ & KUV $06289+3126$ & DA & $\mathrm{sdB}$ & 2 \\
\hline $0713+584$ & GD 294 & DA4 & $\mathrm{sdB}$ & 3 \\
\hline $0713+745$ & Mrk 380 & DA & $\mathrm{QSO}$ & 3 \\
\hline $0720+304$ & SA $51-822 \mathrm{~A}$ & DA2 & $\mathrm{sdB}$ & 5 \\
\hline $0752+412$ & KUV $07528+4113$ & DA? & $\mathrm{sdB}$ & 2 \\
\hline $0852+602$ & PG $0852+602$ & DA2 & $\operatorname{sdB}$ & 3 \\
\hline $0920+366$ & CBS 98 & DA1 & $\operatorname{sdB}$ & 3 \\
\hline $0927+388$ & KUV $09272+3854$ & DA & $\operatorname{sdB}$ & 2 \\
\hline $0930+376$ & KUV $09306+3740$ & DA & $\operatorname{sdB}$ & 2 \\
\hline $0932+396$ & KUV $09327+3937$ & DA & $\operatorname{sdB}$ & 2 \\
\hline $0937+395$ & KUV $09372+3933$ & DA & $\mathrm{sdB}$ & 2 \\
\hline $0939+071$ & PG 0939+072 & DA7 & $\mathrm{MS}(\mathrm{dF})$ & 3 \\
\hline $0943+371$ & KUV $09436+3709$ & DA & $\mathrm{sdB}$ & 2 \\
\hline $0944-090$ & GD 104 & DA1 & $\mathrm{sdO}$ & 6 \\
\hline $0946+381$ & KUV $09467+3809$ & DA & $\mathrm{sdB}$ & 2 \\
\hline $1000+220$ & Ton 1145 & DA6 & $\mathrm{MS}(\mathrm{dF})$ & 3 \\
\hline $1008-179$ & EC $10081-1757$ & DA1 & $\mathrm{sdO}$ & 7 \\
\hline $1036+433$ & Feige 34 & DA & $\mathrm{sdO}$ & 8 \\
\hline $1057+307$ & CSO 64 & DA5 & $\mathrm{MS}(\mathrm{dF})$ & 3 \\
\hline $1121+145$ & PG $1121+145$ & DA1 & $\mathrm{sdB}$ & 9 \\
\hline $1133-528$ & BPM 21065 & DA5 & $\mathrm{MS}(\mathrm{dK})$ & 3 \\
\hline $1137+311$ & CSO 105 & DA5 & $\mathrm{MS}(\mathrm{dF})$ & 3 \\
\hline $1207-032$ & PG $1207-033$ & DA4 & $\mathrm{sdB}$ & 1 \\
\hline $1214+032$ & LP 554-63 & $\mathrm{DA}$ & $\mathrm{dM}$ & 3 \\
\hline $1256+286$ & KUV $12562+2839$ & DA? & $\operatorname{sdB}$ & 2 \\
\hline $1304+313$ & PB 3322 & DA & $\mathrm{sdB}$ & 2 \\
\hline $1357+280$ & CTI $135700.6+280448$ & DA & $\mathrm{MS}(\mathrm{dF})$ & 3 \\
\hline $1412+612$ & HS $1412+6115$ & DAH & sdO & 3 \\
\hline $1412-049$ & PG $1412-049$ & DA & $\mathrm{DA}+\mathrm{dM}$ & 10 \\
\hline $1433-270$ & BPS CS 22874-57 & DA & $\mathrm{sdB}$ & 3 \\
\hline $1501+300$ & $\ldots$ & DA & $\mathrm{MS}(\mathrm{dF})$ & 3 \\
\hline $1509-028.1$ & LP $622-13$ & DA: & $\mathrm{MS}(\mathrm{dF})$ & 3 \\
\hline $1514+033$ & PG $1514+034$ & DAWK & $\mathrm{sdB}$ & 3 \\
\hline $1524+438$ & CBS 246 & DABZ4 & $\mathrm{sdO}$ & 11 \\
\hline $1525+433$ & GD 344 & DA & $\operatorname{sdB}$ & 3 \\
\hline $1532+239$ & Ton 241 & DA & $\operatorname{sdB}$ & 3 \\
\hline $1544+009$ & LB 898 & DA1 & $\mathrm{sdO}$ & 12 \\
\hline $1603+175$ & KUV $16032+1735$ & DAH? & $\mathrm{sdO}+\mathrm{dK}$ & 2 \\
\hline $1611+390$ & KUV 16118+3906 & DA & $\mathrm{sdB}$ & 2 \\
\hline $1616-390$ & Ton 264 & DA4 & $\mathrm{MS}(\mathrm{dF})$ & 3 \\
\hline $1659+442$ & PG $1659+442$ & DA & $\mathrm{sdB}$ & 3 \\
\hline $2122+157$ & PG $2122+157$ & DA & $\operatorname{sdB}$ & 1 \\
\hline $2148+286$ & $\mathrm{BD}+284211$ & DA & $\mathrm{sdO}$ & 13 \\
\hline $2204+071$ & PG 2204+071 & DA & sdOB & 10 \\
\hline $2309+258$ & KUV $23099+2548$ & DA & sdB & 2 \\
\hline $2323-241$ & G275-B16A & DA & $\mathrm{MS}(\mathrm{dF})$ & 3 \\
\hline $2329-291$ & Ton S 102 & DAWK & $\mathrm{sdB}$ & 1 \\
\hline $2333-002$ & PB 5462 & DA2 & $\mathrm{sdO}$ & 14 \\
\hline $2343+043$ & PB 5529 & $\mathrm{DA}$ & $\operatorname{sdB}$ & 3 \\
\hline $2352-255$ & G275-B17B & DA & $\operatorname{sdB}$ & 3 \\
\hline
\end{tabular}

References. (1) Lisker et al. 2005; (2) Limoges \& Bergeron 2010; (3) this work; (4) Kawka et al. 2004; (5) Finlev et al. 1997; (6) Stroeer et al. 2007; (7) Christlieb et al. 2001; (8) Farihi et al.|2005; (9) Green et al.|1986; (10) LBH05; (11) Eisenstein et al.|2006; (12) Catalán et al.|2008; (13) Kilkenny et al.|1988; (14) Hügelemever et al.|2006. 


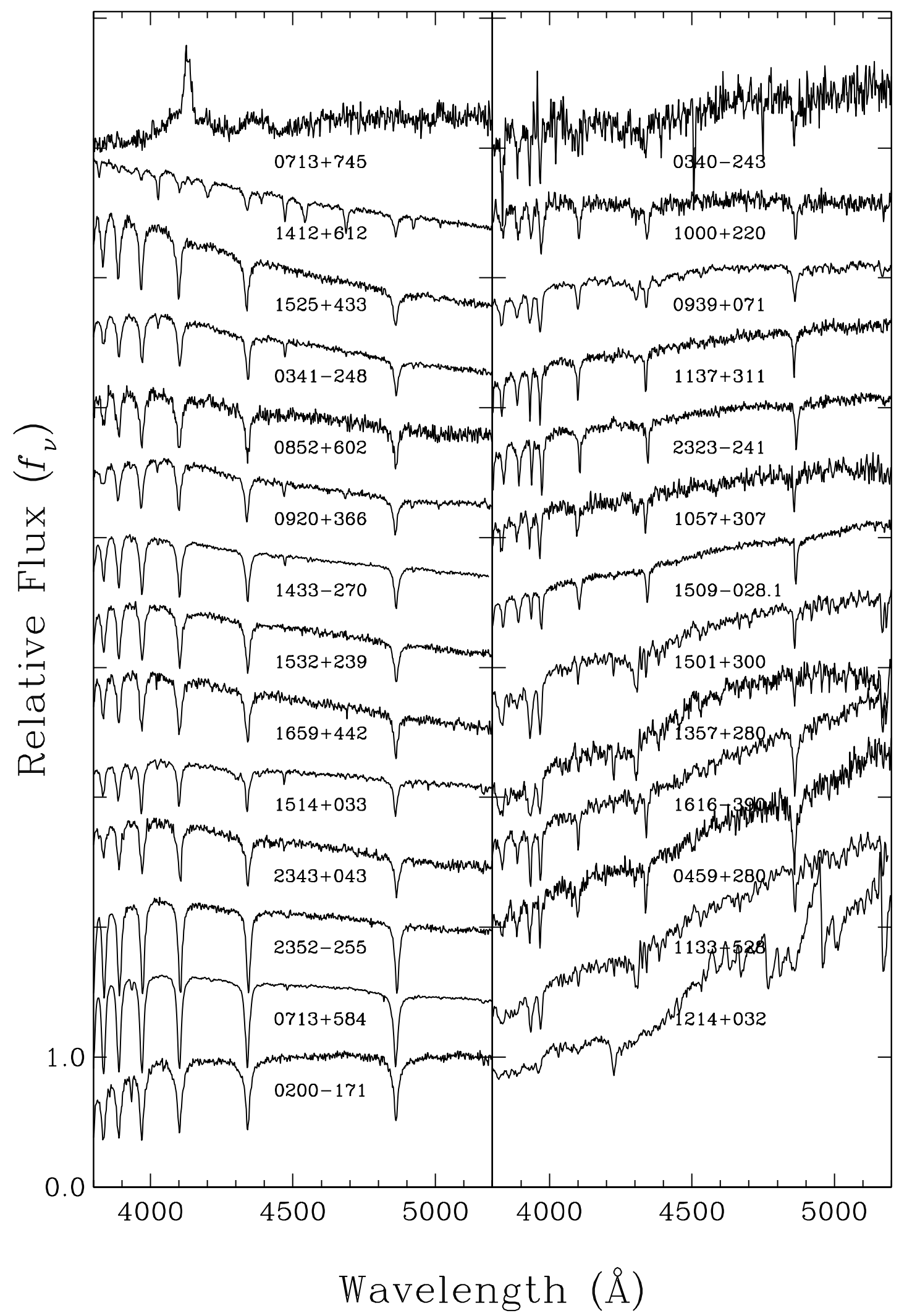

Figure 3. Optical spectra of stars misclassified as DA white dwarfs in MS99 and reported for the first time in this work. The objects are approximately ordered as a function of their slope from upper left to bottom right. The spectra have been normalized to a continuum set to unity and vertically shifted for clarity. 0713+745 (left panel, first object at the top) is a quasar. 


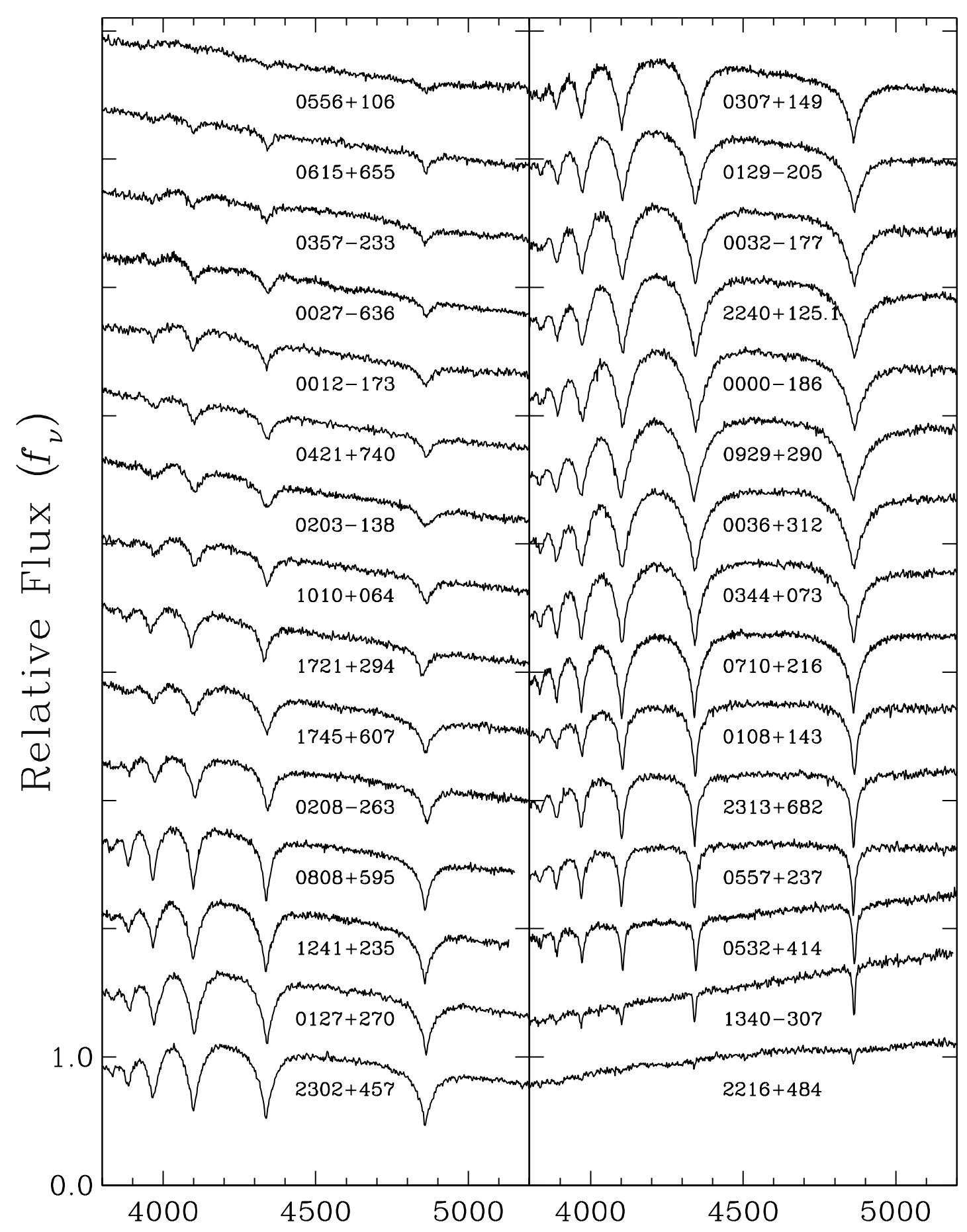

Wavelength $(\AA)$

Figure 4. Optical spectra for a subsample of the DA white dwarfs from our survey of MS99. The spectra are normalized at $4500 \AA$ and shifted vertically for clarity. The effective temperature decreases from upper left to bottom right. All the spectra have $75 \geq \mathrm{S} / \mathrm{N} \geq 65$. 


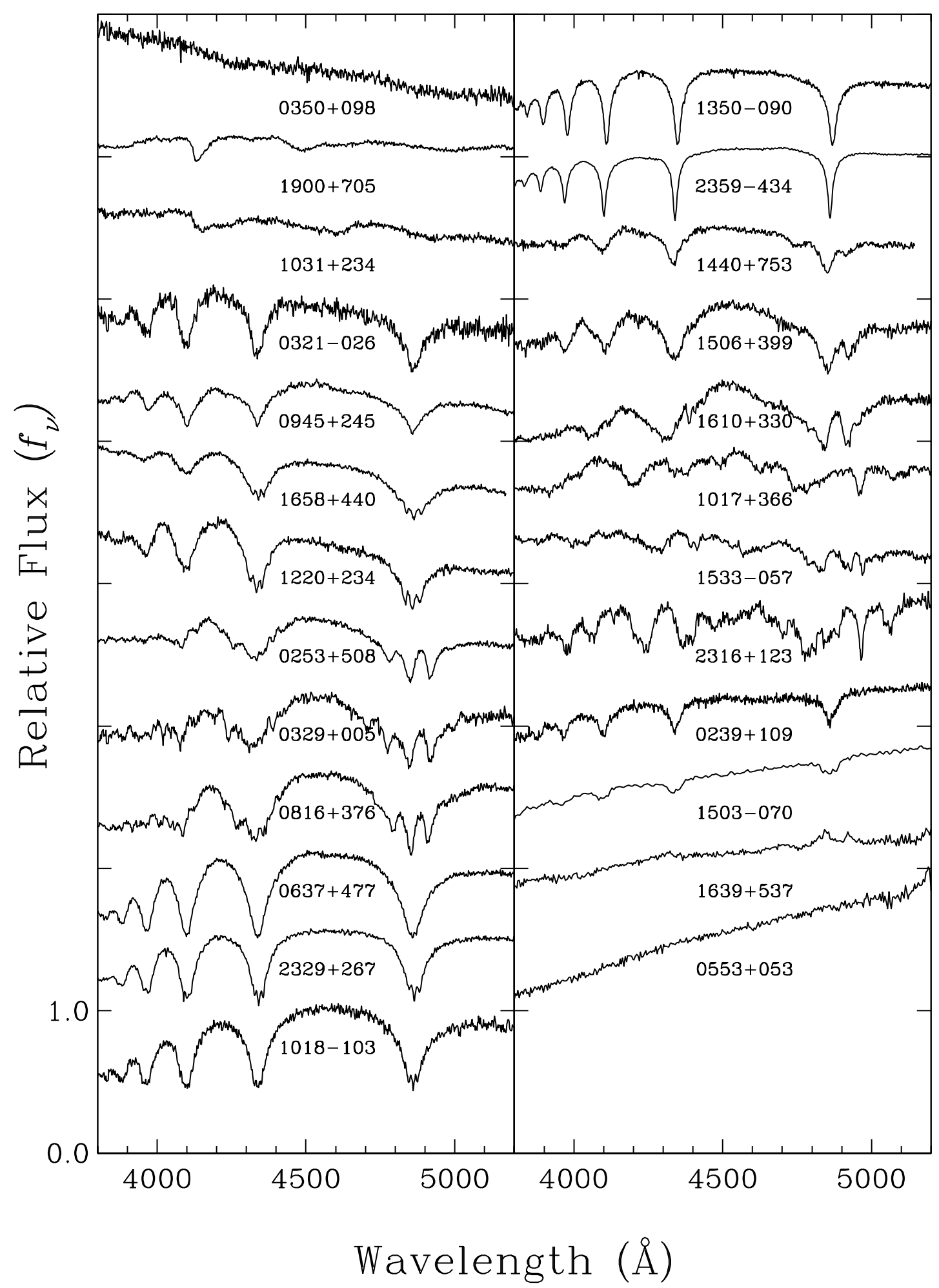

Figure 5. Optical spectra for 25 magnetic (or suspected magnetic) DA white dwarfs from our survey of MS99. The objects are approximately ordered as a function of their slope from upper left to bottom right. The spectra are normalized at $4500 \AA$ and shifted vertically for clarity. 


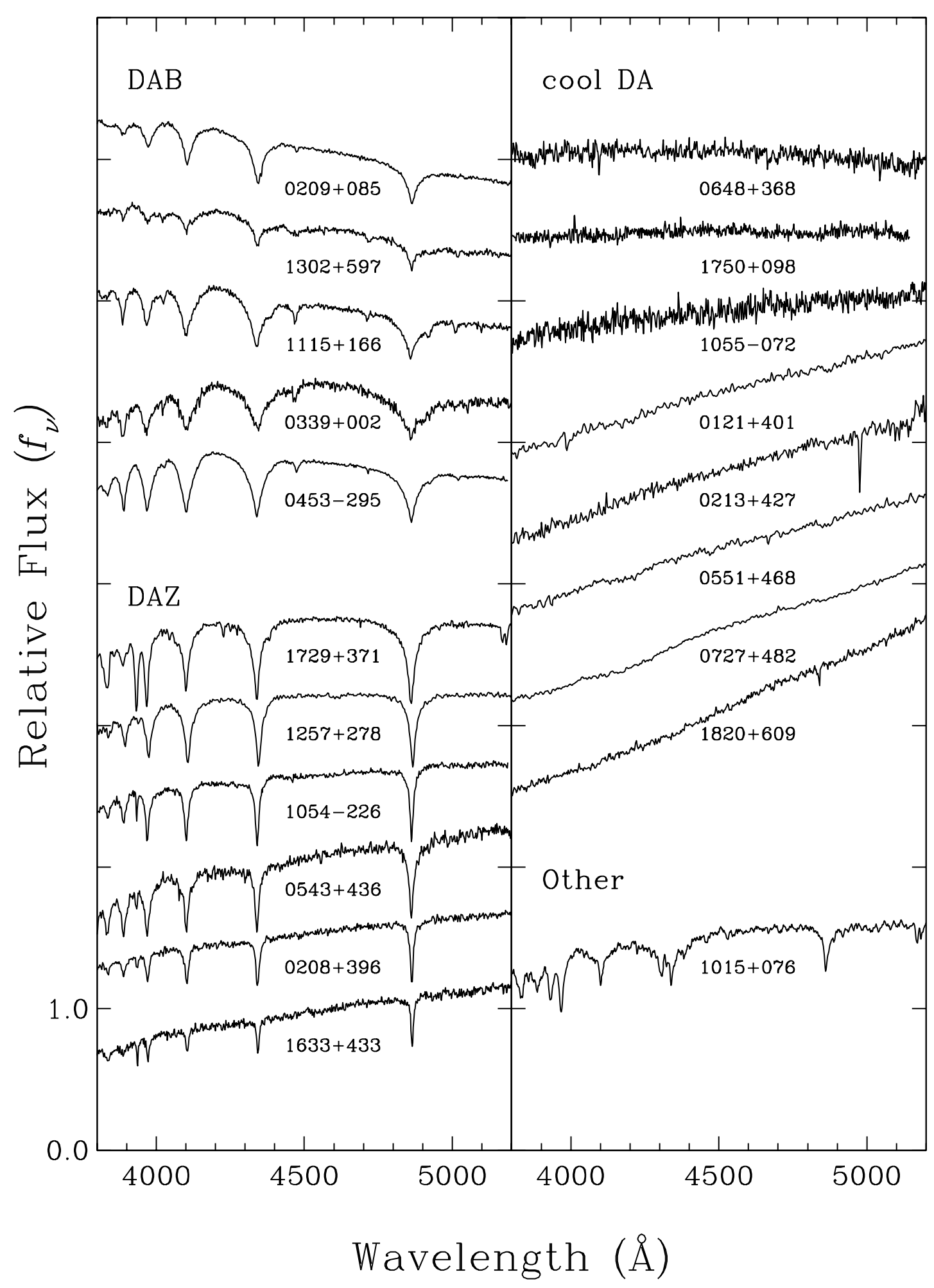

Figure 6. Optical spectra for 20 white dwarfs from our survey of miscellaneous spectral types. The objects are approximately ordered as a function of their slope from upper left to bottom right. The spectra are normalized at $4600 \AA$ and shifted vertically for clarity. 


\section{MODEL ATMOSPHERES}

\subsection{Pure Hydrogen Atmosphere Models}

For the analysis of the vast majority of white dwarfs in our sample, we have employed model atmospheres and synthetic spectra appropriate for DA white dwarfs. These are described at length in LBH05, and references therein. Briefly, these are pure hydrogen, planeparallel model atmospheres where non-local thermodynamic equilibrium (NLTE) effects are explicitly taken into account above $T_{\text {eff }}=20,000 \mathrm{~K}$, and energy transport by convection is included in cooler models following the ML2 $/ \alpha=0.8$ prescription of the mixing-length theory (see Tremblay et al. 2010). The one major upgrade from the models described in LBH05 is that we now use the new and improved Stark broadening profiles of TB09 that account for nonideal effects directly in the line profile calculation. However, since we will wish to compare our results with those of other large surveys, such as SPY, we have also fit our sample using models computed with the older broadening profiles of Lemke (1997), and consequently with the ML2/ $\alpha=0.6$ version of the mixing-length theory (Bergeron et al. 1995), since they were still in use at the time that the SPY analysis was performed. We can thus compare the independent results on a level playing field. Beyond that, performing our analysis with both sets of hydrogen line profiles will also allow us to explore the implications of these improved models on the analysis of DA white dwarfs.

\subsection{Hydrogen Atmosphere Models with CNO}

Although the pure hydrogen models described above are sufficient in most cases, some white dwarfs present unique challenges that require somewhat more elaborate models. This is the case with the hot DAO white dwarfs as well as the hot DA stars that exhibit the so-called Balmer line problem (DA+BP), which manifests itself as an inability to fit all the Balmer lines simultaneously with consistent atmospheric parameters. Though the models used to properly fit the spectra of these stars are presented and described at length in Gianninas et al. (2010), for the benefit of the reader, we briefly explain them here. The above-mentioned models have all been computed using the TLUSTY atmosphere code and the accompanying spectrum synthesis code SYNSPEC, both developed by Hubeny \& Lanz (1995). The models are computed in NLTE and include carbon, nitrogen, and oxygen (CNO) at solar abundances (Asplund et al. 2005). As Werner (1996) demonstrated, the inclusion of CNO reduces the temperature in the upper layers of the atmosphere, which in turn produces deeper cores for the hydrogen Balmer lines, thus overcoming the Balmer line problem. As clearly stated by Gianninas et al. (2010), CNO acts as a proxy for all metals that may be present in the atmosphere. As such, the inclusion of CNO at solar abundances should in no way be considered as a determination of the abundance of CNO in these stars. The model atmospheres used for the DAO and DA+BP stars are identical, save for the inclusion of helium in the DAO models in order to reproduce the He II $\lambda 4686$ line.

\subsection{Helium Atmosphere Models}

In Figure 6] we presented three DA+DB binary systems that are included in our sample. In order to prop-
Table 2

Breakdown of Spectral Types

\begin{tabular}{cccccccc}
\hline \hline DA & DAO & DAZ & DAB & DAmag & DA+dM & Cool DA & Total \\
\hline 1171 & 30 & 6 & 5 & 25 & 47 & 8 & 1292 \\
\hline
\end{tabular}

erly fit the spectra of these stars, we will need to combine our pure hydrogen models with pure helium models. The helium models we have employed are described in Bergeron et al. (2011), which are based on the updated grid of white dwarf models described in TB09 in which the improved Stark profiles of neutral helium of Beauchamp et al. (1997) have been incorporated. These models are comparable to those described by Beauchamp (1995) and Beauchamp et al. (1996), with the exception that at low temperatures $\left(T_{\text {eff }}<10,800 \mathrm{~K}\right)$, the free-free absorption coefficient of the negative helium ion of John (1994) is now used. The models are in local thermodynamic equilibrium (LTE) and include convective energy transport within the mixing-length theory. As in the analysis of Beauchamp et al. (1999), the parameterization described as ML2 $/ \alpha=1.25$ is implemented.

\section{SPECTROSCOPIC ANALYSIS}

\subsection{Fitting Technique}

The method used for fitting the observations relies on the so-called spectroscopic technique developed by Bergeron et al. (1992), which has been refined by Bergeron et al. (1995) and more recently by LBH05, and includes the Balmer lines from $\mathrm{H} \beta$ to $\mathrm{H} 8$. The first step is to normalize the flux from an individual line, in both observed and model spectra, to a continuum set to unity at a fixed distance from the line center. The comparison with model spectra, which are convolved with the appropriate Gaussian instrumental profile (3, 6, 9, and $12 \AA$ ), is then carried out in terms of these line shapes only. The most sensitive aspect of this fitting technique is to define the continuum of the observed spectra. The approach is slightly different depending on the temperature range in question. For stars in the interval $16,000 \mathrm{~K} \gtrsim T_{\text {eff }} \gtrsim$ $9000 \mathrm{~K}$, where the Balmer lines are at their strongest, the normalization procedure involves the use of several pseudo-Gaussian profiles (Bergeron et al. 1995, and references therein). This procedure is quite reliable as the sum of the pseudo-Gaussian profiles represents a good approximation to the observed Balmer lines. Outside of this temperature range, the method becomes more unstable as the continuum between the Balmer lines becomes essentially linear. Consequently, for stars with $T_{\text {eff }}>$ $16,000 \mathrm{~K}$ and $T_{\text {eff }}<9000 \mathrm{~K}$, we rely on our synthetic spectra to reproduce the observed spectrum, including a wavelength shift, as well as several order terms in $\lambda$ (up to $\lambda^{6}$ ) using the nonlinear least-squares method of Levenberg-Marquardt (Press et al. 1986). The normal points are then fixed at the points defined by this smooth model fit. Note that the values of $T_{\text {eff }}$ and $\log g$ at this stage are meaningless since too many fitting parameters are used, and the model just serves as a smooth fitting function to define the continuum of the observed spectrum. Once the lines are normalized to a continuum set 


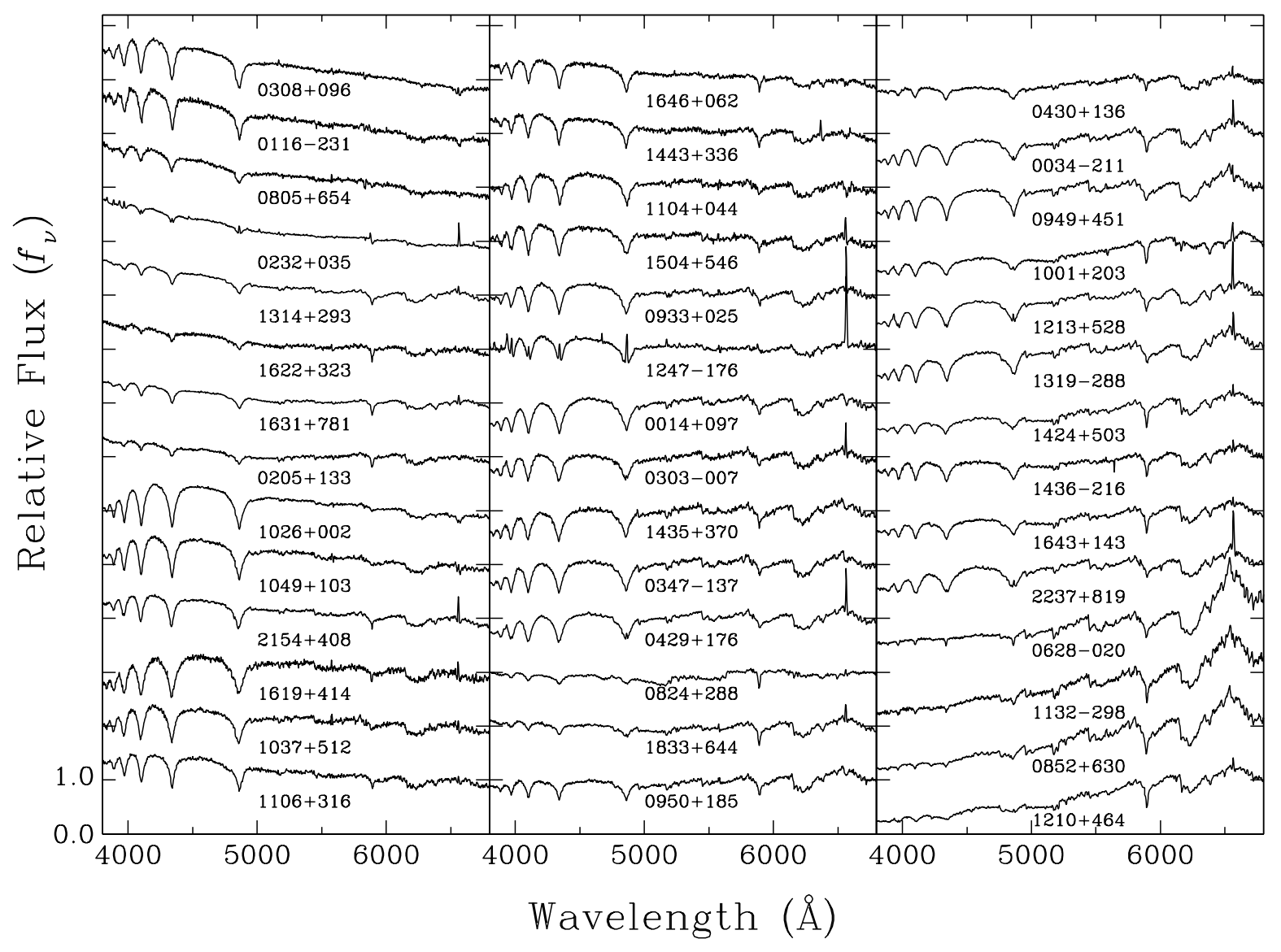

Figure 7. Optical spectra for $42 \mathrm{DA}+\mathrm{dM}$ binary systems covering the range $3500-7000 \AA$. The spectra are normalized to unity at 6500 $\AA$ and shifted vertically for clarity.

to unity, we use our grid of model spectra to determine $T_{\text {eff }}$ and $\log g$ in terms of these normalized profiles only. Our minimization technique again relies on the nonlinear least-squares method of Levenberg-Marquardt, which is based on a steepest descent method. Sample fits of 20 DA white dwarfs, covering almost the entire temperature range of our sample are displayed in Figure 8. We see that the combination of high $\mathrm{S} / \mathrm{N}$ spectra with the procedures described above allow us to achieve a proper normalization in each case. Hence, we are able to obtain an excellent agreement between the observed and predicted line profiles for nearly every star. The spectrum of one star, $0342+374$, features emission lines from its associated planetary nebula. These emission lines can interfere with our normalization procedure but also with our fitting technique and so we simply exclude the affected spectral ranges from both the normalization and fitting procedure.

\subsection{Effects of New Stark Profile Calculations}

As we have mentioned, in this study we will be passing from the use of the old Lemke (1997) profiles to the new TB09 Stark broadening profiles that include non-ideal effects directly in the line profile calculation. In doing so, it is important to understand what effects these new profiles will have on our atmospheric parameter determinations. TB09 explored some of these differences (see their Figure 12) using the PG sample of LBH05, but this limited them to a particular range of $T_{\text {eff }}$ whereas we can examine the effects along virtually the entire white dwarf cooling sequence.

In Figure 9] we present the comparison between the atmospheric parameters measured using the older Lemke (1997) and the new TB09 Stark profiles for the 1151 DA stars in our sample. In the lower panel, we see that the correlation between the old and new values of $T_{\text {eff }}$ is very tight although the overall trend is to higher temperatures. This effect is somewhat more evident at higher $T_{\text {eff }}$. In the upper panel of Figure 9, we compare the old and new values of surface gravity. Unlike with the values of $T_{\text {eff }}$ we note here a much more obvious shift toward higher measurements of $\log g$ when using the new TB09 Stark broadening profiles. These results are consistent with those presented in TB09.

In Figure [10, we examine how these differences in the measured atmospheric parameters translate to the determination of the mass distribution as a function of $T_{\text {eff }}$. Using the old profiles, we see in the top panel of Figure 10 that besides the high-log $g$ problem at lower temperatures (Tremblay et al. 2010, and Section 5.5 below), the mass distribution dips near $13,000 \mathrm{~K}$. On the other hand, the new profiles seem to even out the mass distribution with the bulk of the stars showing masses between $0.55 M_{\odot}$ and $0.7 M_{\odot}$ before the high-log $g$ problem takes 


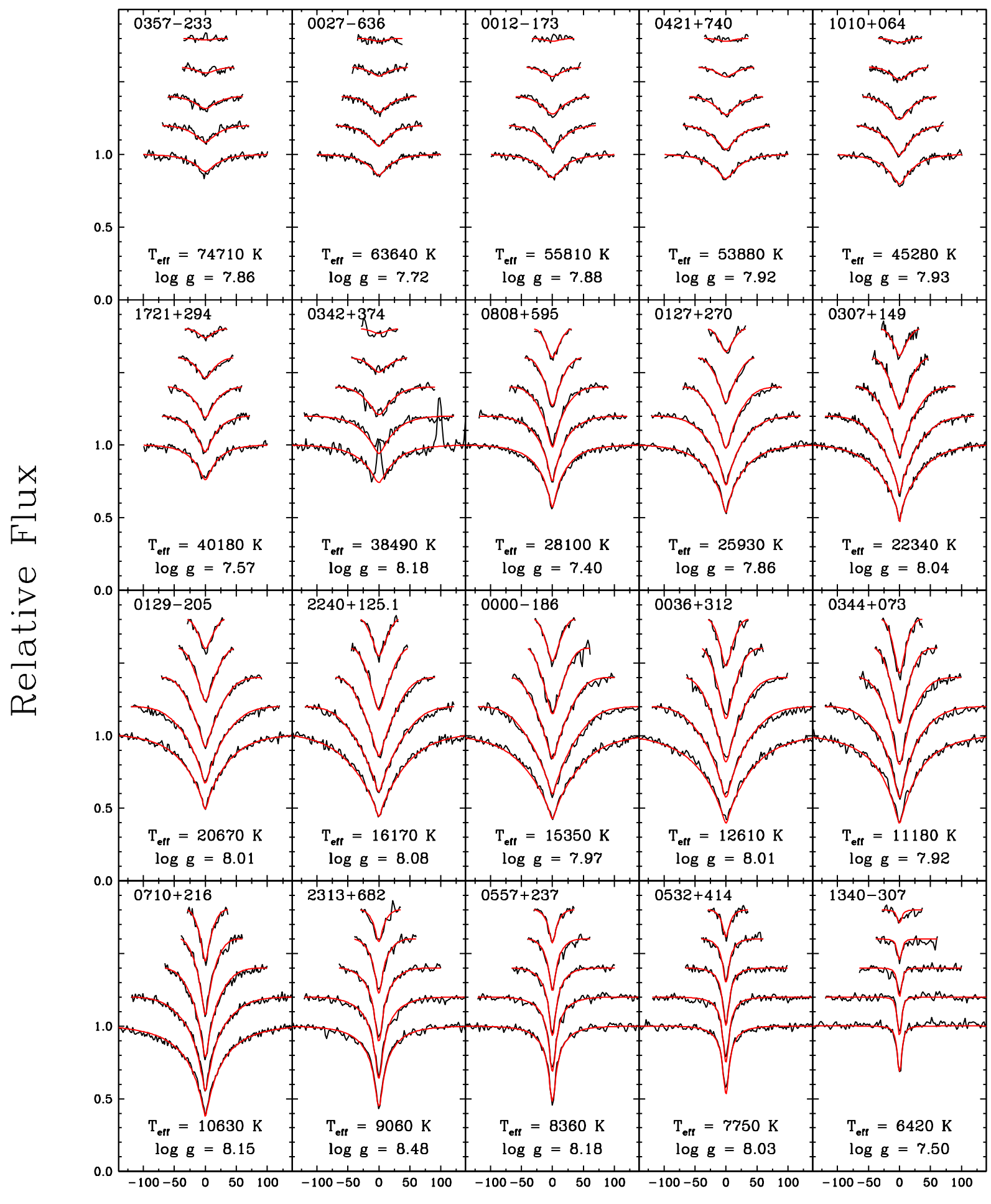

$\Delta \lambda(\AA)$

Figure 8. Model fits (red) to the individual Balmer line profiles (black) of a sample of DA white dwarfs taken from Figure 4 in order of decreasing effective temperature. The lines range from $\mathrm{H} \beta$ (bottom) to $\mathrm{H} 8$ (top), each offset by a factor of 0.2 . The best-fit values of $T_{\text {eff }}$ and $\log g$ are indicated at the bottom of each panel. 

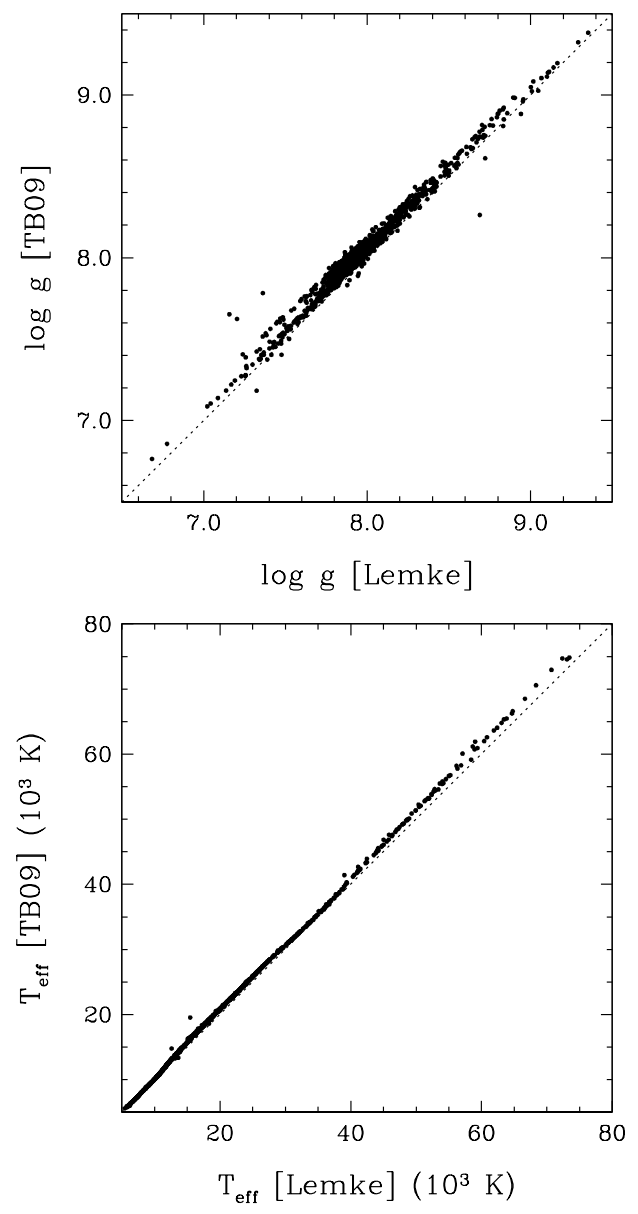

Figure 9. Comparison of $\log g$ (top) and $T_{\text {eff }}$ (bottom) values derived from fits using the improved models of TB09 and the profiles of Lemke (1997) for 1151 DA white dwarfs. The dotted lines represent the 1:1 correlation.

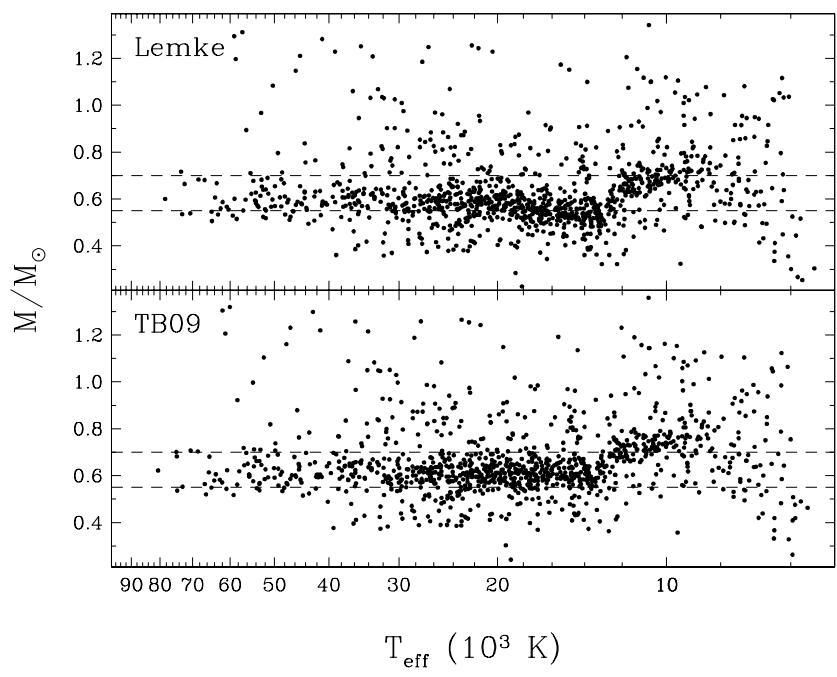

Figure 10. Mass vs. $T_{\text {eff }}$ distribution for 1151 DA white dwarfs in our sample using the Stark broadening profiles of Lemke (1997; top) and the improved calculations of TB09 (bottom). Lines of constant mass at $0.55 M_{\odot}$ and $0.7 M_{\odot}$ are shown as a reference.

hold at lower $T_{\text {eff }}$. As such, the new profiles permit an improved determination of the mass distribution as a func-

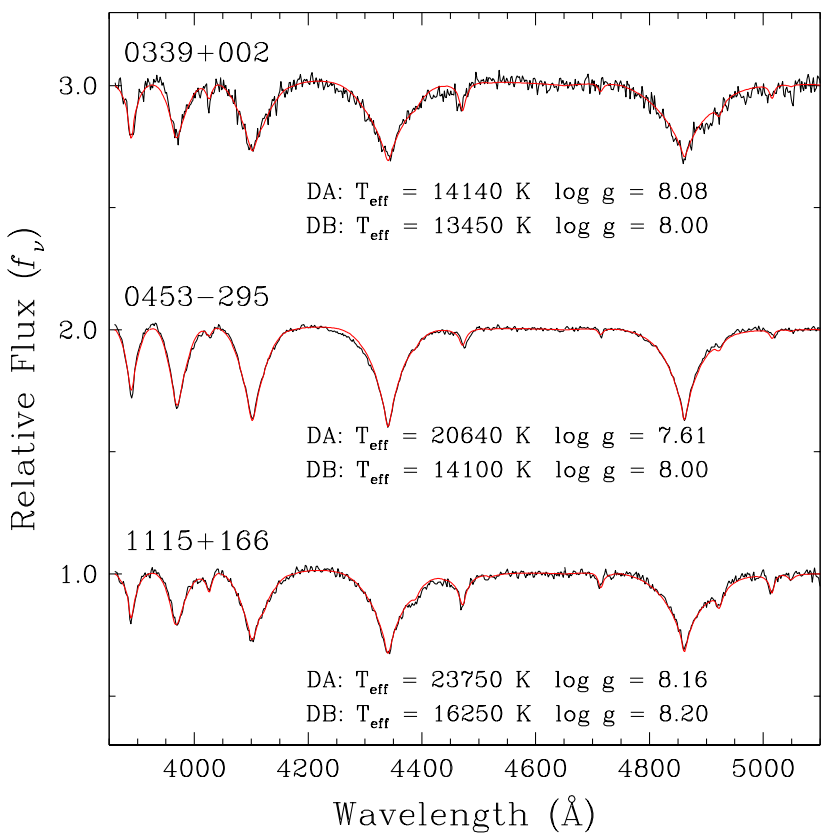

Figure 11. Model fits (red) to the optical spectra (black) for the three DA+DB binary systems in our sample. The atmospheric parameters for each solution are given in the figure. Both the observed and theoretical spectra are normalized to a continuum set to unity and the spectra are vertically shifted for clarity.

tion of $T_{\text {eff }}$ over the older models that employ Lemke's Stark broadening profiles. Specifically, the TB09 profiles produce a relatively constant mass distribution down to about $13,000 \mathrm{~K}$ where the high-log $g$ problem begins to manifest itself.

In the following sections, we will focus on the spectroscopic analysis of all the objects and systems that require particular attention. These include white dwarfs of spectral types DAB, DAZ, DA+dM binary systems, magnetic white dwarfs, and a selection of other unique objects that demanded a more detailed analysis. The motivation here is to ensure that we determine the most accurate atmospheric parameters possible for each star. With that in mind, we also note that from this point forward, we will be using the new Stark profiles of TB09 exclusively.

\subsection{DAB White Dwarfs}

In Figure 6, we displayed five white dwarfs classified as DAB stars. In other words, besides the hydrogen Balmer lines, the spectra of these stars also feature lines due to neutral helium. For three of these objects $(0339+002,0453-295,1115+166)$, previous analyses have shown that fits assuming a single star do not produce satisfactory results. In contrast, fits performed under the assumption that the spectrum is that of a $\mathrm{DA}+\mathrm{DB}$ double-degenerate binary system have proven successful (Limoges \& Bergeron 2010; Wesemael et al. 1994; Bergeron \& Liebert 2002, respectively). With that in mind, we proceed to fit the spectra of these systems by combining our usual pure hydrogen models with the pure helium models described in Section 3.3 . When fitting DA+DB model spectra, the total flux of the system is obtained from the sum of the monochromatic Eddington fluxes of the individual components, weighted by their respective radius. The stellar radii are ob- 


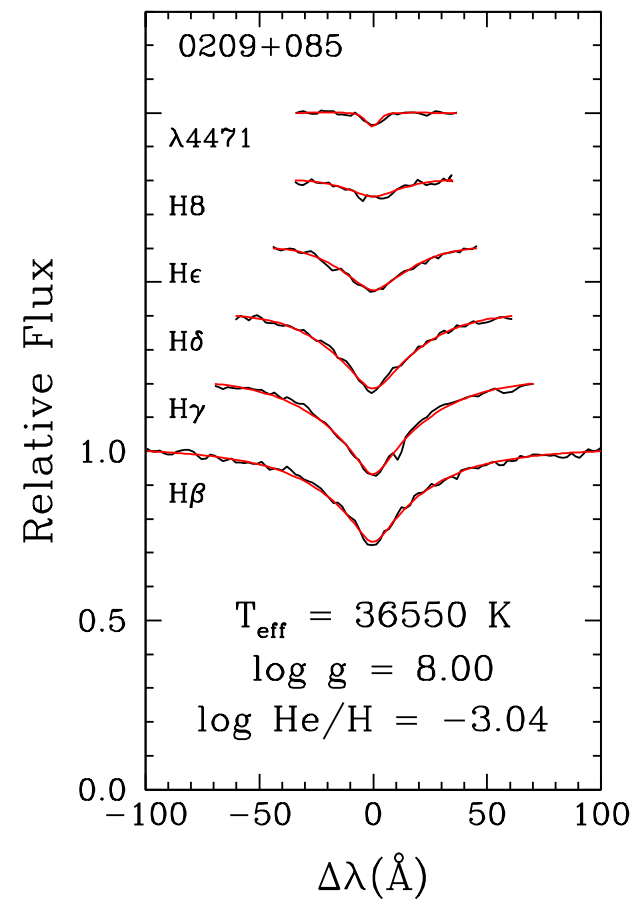

Figure 12. Model fits (red) to the hydrogen Balmer lines and the He I $\lambda 4471$ line in the observed optical spectrum (black) of the DAB white dwarf HS 0209+0832. The lines range from $\mathrm{H} \beta$ (bottom) to $\mathrm{H} 8$ in addition to He I $\lambda 4471$ (top), each offset by a factor of 0.2 . The best-fit values of $T_{\text {eff }}, \log g$, and $\log \mathrm{He} / \mathrm{H}$ are indicated at the bottom of the figure.

tained from evolutionary models similar to those described in Fontaine et al. (2001) but with C/O cores, $q(\mathrm{He}) \equiv \log M_{\mathrm{He}} / M_{\star}=10^{-2}$ and $q(\mathrm{H})=10^{-4}$, which are representative of hydrogen atmosphere white dwarfs, and $q(\mathrm{He})=10^{-2}$ and $q(\mathrm{H})=10^{-10}$, which are representative of helium atmosphere white dwarfs.

The fitting technique for these $\mathrm{DA}+\mathrm{DB}$ systems first requires that we normalize both the observed and synthetic spectra to a continuum set to unity. The calculation of $\chi^{2}$ is then carried out in terms of these normalized line profiles only. The atmospheric parameters, $T_{\text {eff }}$ and $\log g$, for the DA white dwarf and $T_{\text {eff }}$ for the DB white dwarf are considered free parameters. We set $\log g=$ 8.0 for the DB components in $0339+002$ and $0453-295$ since the helium lines are rather weak and are not especially sensitive to the surface gravity. The helium lines are comparatively stronger in $1115+166$ so for the fit of this star, we allow $\log g$ to vary.

Our best fits to the spectra of $0339+002,0453-295$, and $1115+166$ are shown in Figure 11, We obtain excellent fits for all three systems with the fit for $1115+166$ being exceptionally good. For $0339+002$, we obtain higher values of $T_{\text {eff }}$ and $\log g$ for the DA component as compared to the results of Limoges \& Bergeron (2010) with a comparable value of $T_{\text {eff }}$ for the $\mathrm{DB}$ component. This is not an altogether surprising result for the DA star since we are using the new profiles of TB09 in our analysis. With regards to $0453-295$, we obtain a substantially higher $T_{\text {eff }}$ and $\log g$ for the DA component as compared to Wesemael et al. (1994) and also a higher $T_{\text {eff }}$ for the DB white dwarf. However, the models used in their anal- ysis are considerably outdated and they also performed a four parameter fit allowing the surface gravity of the DB star to vary. Finally, we get somewhat higher values of $T_{\text {eff }}$ and $\log g$ than Bergeron \& Liebert (2002) for the DA component but identical parameters for the DB.

The case of HS $0209+0832$ is different from the above three systems as it is a true DAB white dwarf, as first reported by Jordan et al. (1993). In other words, it is a single star with both hydrogen and neutral helium lines present in its optical spectrum. Furthermore, like GD 323, this star presents neutral helium lines that are variable, in particular the He I $\lambda 4471$ line (Heber et al. 1997). However, contrary to GD 323, Wolff et al. (2000) were able to explain the variability as resulting from the accretion of pockets of interstellar matter of varying densities leading to different accretion rates over the course of time. We show in Figure 12 the best fit to our optical spectrum of HS 0209+0832. Besides the usual Balmer lines, we also fit the He I $\lambda 4471$ line in order to measure the helium abundance. To perform the fit, we have used the grid of homogeneously mixed $\mathrm{H} / \mathrm{He}$ models described by Gianninas et al. (2010) for DAO white dwarfs, with some modifications. Namely, we have extended the grid down to $T_{\text {eff }}=30,000 \mathrm{~K}$. However, we do not include $\mathrm{CNO}$ in the models as there is no evidence of the Balmer line problem in HS 0209+0832.

Our new determinations $\left(T_{\text {eff }}=36,550, \log g=8.00\right)$ are quite comparable to the previous measurements of Heber et al. (1997, $\left.T_{\text {eff }}=36,100, \log g=7.91\right)$ and Wolff et al. (2000, $\left.T_{\text {eff }}=35,500, \log g=7.90\right)$ with our higher values likely due to the use of the new TB09 Stark broadening profiles. Conversely, we determine a much lower helium abundance of $\log \mathrm{He} / \mathrm{H}=-3.04$ as compared to the values compiled in Table 2 of Wolff et al. (2000) that hover around $\log \mathrm{He} / \mathrm{H} \sim-2.0$ with the lowest value at $\log \mathrm{He} / \mathrm{H}=-2.32$ (Heber et al. 1997). It is important to note that our spectrum was obtained in 2002 December, more than three years after the last observation listed in Table 2 of Wolff et al. (2000). Furthermore, Wolff et al. state that "A lower rate would reduce the abundance within a few months due to the short diffusion timescale". Hence, the most logical explanation for our considerably lower helium abundance determination is that in the intervening time, HS 0209+0832 traversed a region of space where the helium density was lower, leading to a reduced accretion rate.

Finally, we do not attempt to analyze GD 323. Indeed, to date there has not been a model that has been able to achieve a satisfactory fit to the optical spectrum of GD 323 despite many attempts throughout the years. We refer the reader to Pereira et al. (2005) for a comprehensive summary of the various models that have been elaborated over the years in order to explain both the optical spectrum of GD 323, and the observed spectral variability.

\subsection{DAZ White Dwarfs}

Our sample contains the six DAZ white dwarfs shown in Figure 6. With the exception of GD 362, we fit the remaining five DAZ white dwarfs to obtain $T_{\text {eff }}, \log g$, as well as the calcium abundance. In order to do so, we computed a small grid of synthetic spectra based on our grid of pure hydrogen atmospheres and added calcium in the calculation of the synthetic spectrum only, as de- 


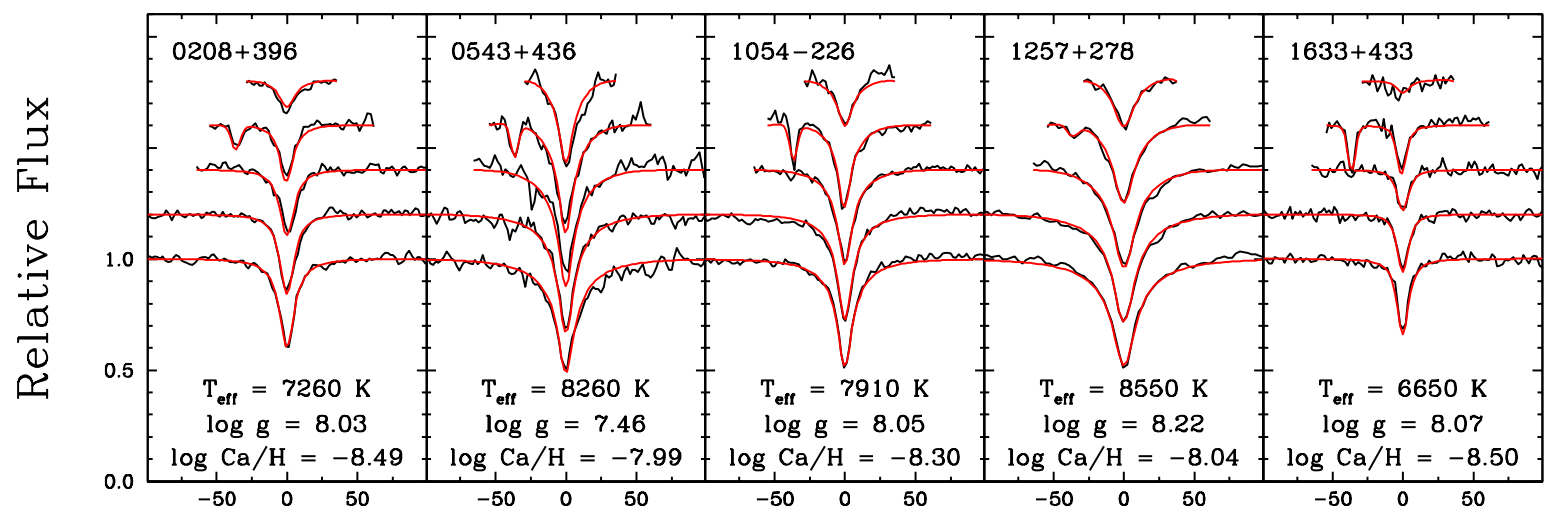

$\Delta \lambda(\AA)$

Figure 13. Model fits (red) to the hydrogen Balmer lines and the Ca H \& K lines in the observed optical spectra (black) of five DAZ white dwarfs. The lines range from $\mathrm{H} \beta$ (bottom) to $\mathrm{H} 8$ (top), each offset by a factor of 0.2 . We note that the Ca $\mathrm{H}$ line is blended with $\mathrm{H} \epsilon$. The best-fit values of $T_{\text {eff }}, \log g$, and $\log \mathrm{Ca} / \mathrm{H}$ are indicated at the bottom of each panel.

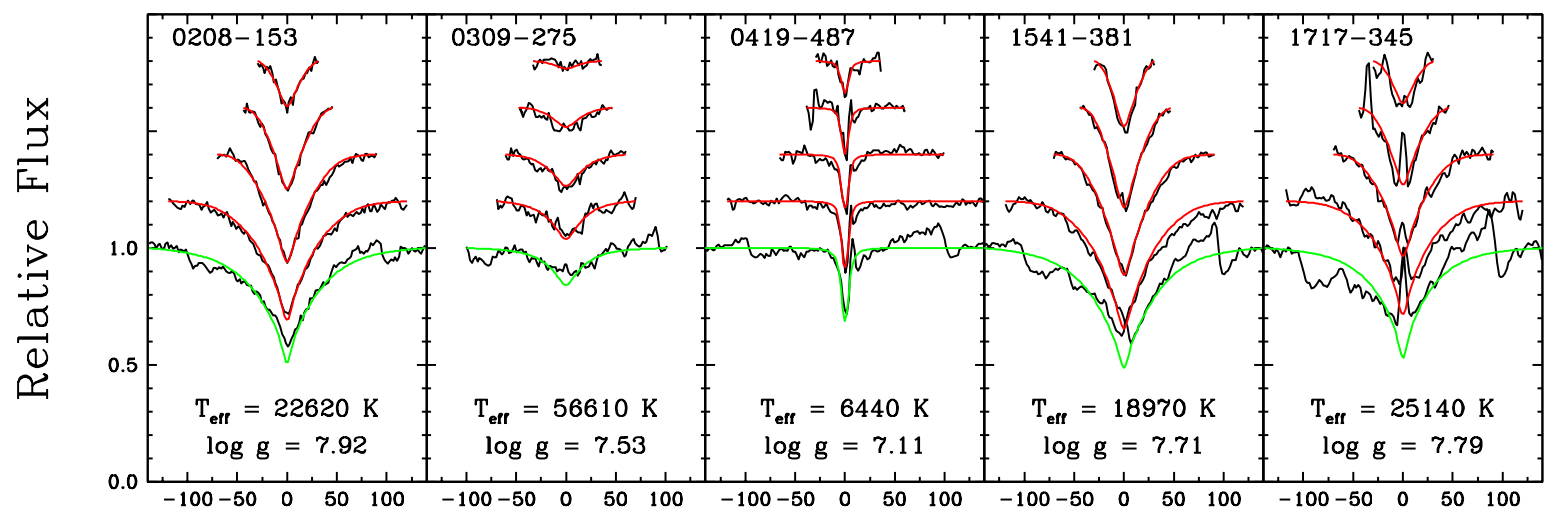

$\Delta \lambda(\AA)$

Figure 14. Same as Figure 8 for five DA+dM binary systems for which we do not have red spectra. In these cases, H $\beta$ (green) is omitted from the fitting procedure as the only contaminated spectral line.

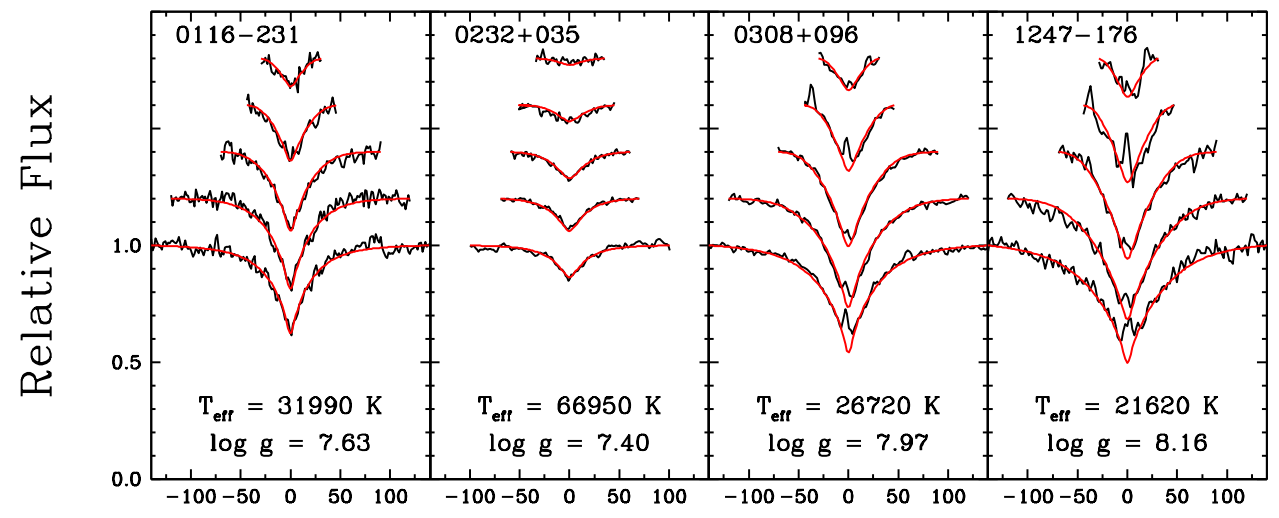

$\Delta \lambda(\AA)$

Figure 15. Same as Figure 8 for four additional DA+dM binary systems where little to no contamination is detected in their blue spectra. 
scribed in Billères et al. (1997). The inclusion of calcium in the calculation of the synthetic spectrum alone, and not in the model atmosphere itself, is justified since the trace amounts of calcium will have no effect whatsoever on the thermodynamic structure of the atmosphere (see Gianninas et al. 2004). Our grid covers $T_{\text {eff }}$ from 6000 to $9000 \mathrm{~K}$ in steps of $500 \mathrm{~K}, \log g$ from 7.0 to 9.5 in steps of 0.5 dex and $\log \mathrm{Ca} / \mathrm{H}$ from -7.0 to -9.5 in steps of 0.5 dex. Although our standard spectroscopic technique works well even without the inclusion of calcium in the synthetic spectra, the fact is that the calcium $\mathrm{H}$ line (at $3968 \AA$ ) is blended with $\mathrm{H} \epsilon$ (at $3970 \AA$ ). Since the upper Balmer lines are especially sensitive to the surface gravity, any failure to properly model the line profile would lead to a less precise measurement of $\log g$. Therefore, we are compelled to include the calcium lines in our spectra to avoid this potential source of uncertainty.

Our best fits of the five DAZ stars using the above grid are displayed in Figure 13. We have employed essentially the same fitting procedure used for our regular DA white dwarfs but here we have three free parameters: $T_{\text {eff }}, \log g$, and the calcium abundance. Consequently, we have extended the fitting region around $\mathrm{H} \epsilon$ blueward in order to properly include the $\mathrm{Ca} \mathrm{K}$ line in the fit. We can see that we are able to reproduce the calcium lines rather well in all cases. Furthermore, we can compare the atmospheric parameters of the three known DAZ stars, G74-7 (0208+396), LTT 13742 (1257+278), and G180$43(1633+433)$, to previous determinations. Specifically, Billères et al. (1997) obtained $T_{\text {eff }}=7260 \mathrm{~K}, \log g=8.03$, and $\log \mathrm{Ca} / \mathrm{H}=-8.80$ for G74-7, and Zuckerman et al. (2003) obtained $T_{\text {eff }}=8481 \mathrm{~K}, \log g=7.90$, and $\log$ $\mathrm{Ca} / \mathrm{H}=-8.07$ for LTT 13742 , and $T_{\text {eff }}=6569 \mathrm{~K}, \log g$ $=8.08$, and $\log \mathrm{Ca} / \mathrm{H}=-8.63$ for G180-43. These values are quite comparable to the values we measure here. Indeed, some variation is expected considering our models contain several improvements over the ones used in the previous analyses. With respect to the two newly identified DAZ stars, G96-53 (0543+436) and LP 849-31 (1054-226), their atmospheric parameters seem to suggest that they are rather typical of DAZ white dwarfs within the same temperature range (see Figure 5 of Zuckerman et al. 2003).

\subsection{DA+dM Binary Systems}

As stated in Section 2, we have a total of $47 \mathrm{DA}+\mathrm{dM}$ binary systems in our sample. For nine of those systems, we employ our standard fitting technique, as described above. The fits for these nine systems are displayed in Figures 14 and 15. Of these nine, five are the DA+dM systems, listed earlier, for which we were unable to secure red spectra (see Section 2). In these cases, we simply omit $\mathrm{H} \beta$, the only contaminated Balmer line, from our fitting procedure and fit only the Balmer lines from $\mathrm{H} \gamma$ to H8. We see in Figure 14 that we are able to achieve a more than satisfactory fit to the remaining four Balmer lines. On the other hand, the remaining four $\mathrm{DA}+\mathrm{dM}$ systems, shown in Figure [15, display little to no contamination from their companion in their blue spectra and we obtain excellent fits to their observed line profiles without the need to omit any lines. First, the red spectrum of $0116-231$ shows virtually no contamination from a companion, as we can see in the left panel of Fig-

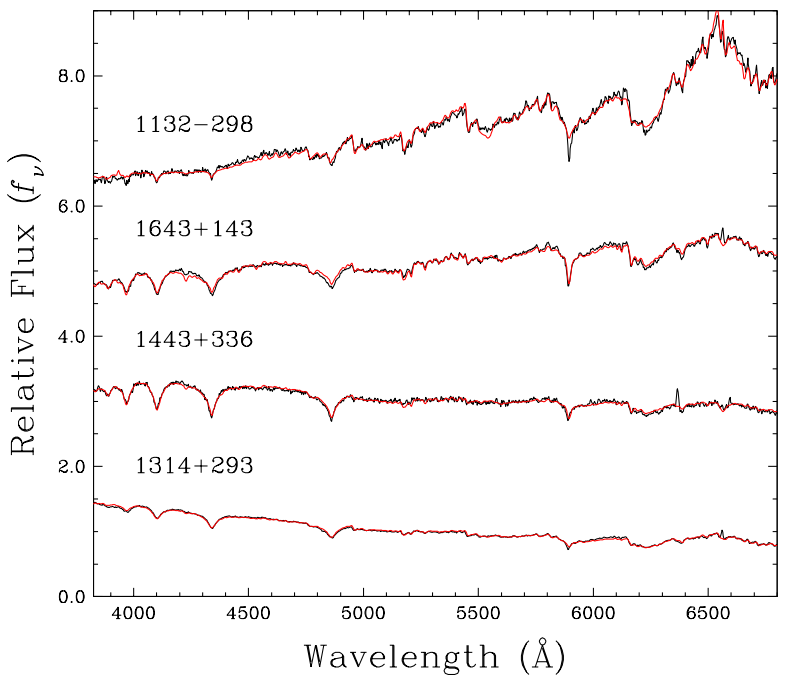

Figure 16. Best fits of the observed red spectra (black) for four of our DA $+\mathrm{dM}$ binary systems using our combination of synthetic DA spectra and $\mathrm{dM}$ spectral templates (red). The spectra are normalized to unity at $5100 \AA$ and shifted vertically for clarity.

ure 7 Similarly, the red spectrum of $0308+096$ is also devoid of contamination except for emission at $\mathrm{H} \alpha$. In contrast, the blue spectrum $0232+035$ seems devoid of any contamination while its red spectrum has emission lines in the cores of all the Balmer lines. Finally, the blue spectrum of 1247-176 shows weak emission for some of the Balmer lines while we see strong emission in the core of all the Balmer lines in its red spectrum (see the middle panel of Figure 7). In the first two cases, there is insufficient contamination in the blue spectra to warrant using the procedure described below to subtract the contamination due to the secondary. In the latter two cases, the red spectrum is considerably more contaminated than the blue spectrum. As such, our procedure would likely only make matters worse. Furthermore, these seemingly contradictory detections and non-detections of emission lines from the $\mathrm{M}$ dwarf secondary in the latter two cases are likely due to the fact that the red and blue spectra were obtained at different phases of the binary orbit. Finally, as described above for $0342+374$, we exclude the center of each Balmer line from the fitting procedure for $0308+096,1247-176$, and $1717-345$, as they are contaminated by emission lines from their $\mathrm{M}$ dwarf companions.

For the remaining 38 systems, we will take advantage of the red spectra we obtained (see Figure 7) in order to remove the contamination due to the presence of the $\mathrm{M}$ dwarf. There have been several studies in the last few years that have analyzed DA+dM systems from the SDSS (Silvestri et al. 2006; Heller et al. 2009; Rebassa-Mansergas et al. 2010). One important distinction between these studies and our own is that in the above cases the authors were interested in recovering both the spectrum of the white dwarf and the spectrum of the $\mathrm{M}$ dwarf in an effort to study the system as a whole and obtain atmospheric parameters for both stars. In contrast, we are only interested in obtaining a more accurate measurement of the parameters for the DA star.

Our fitting procedure for these systems combines the 


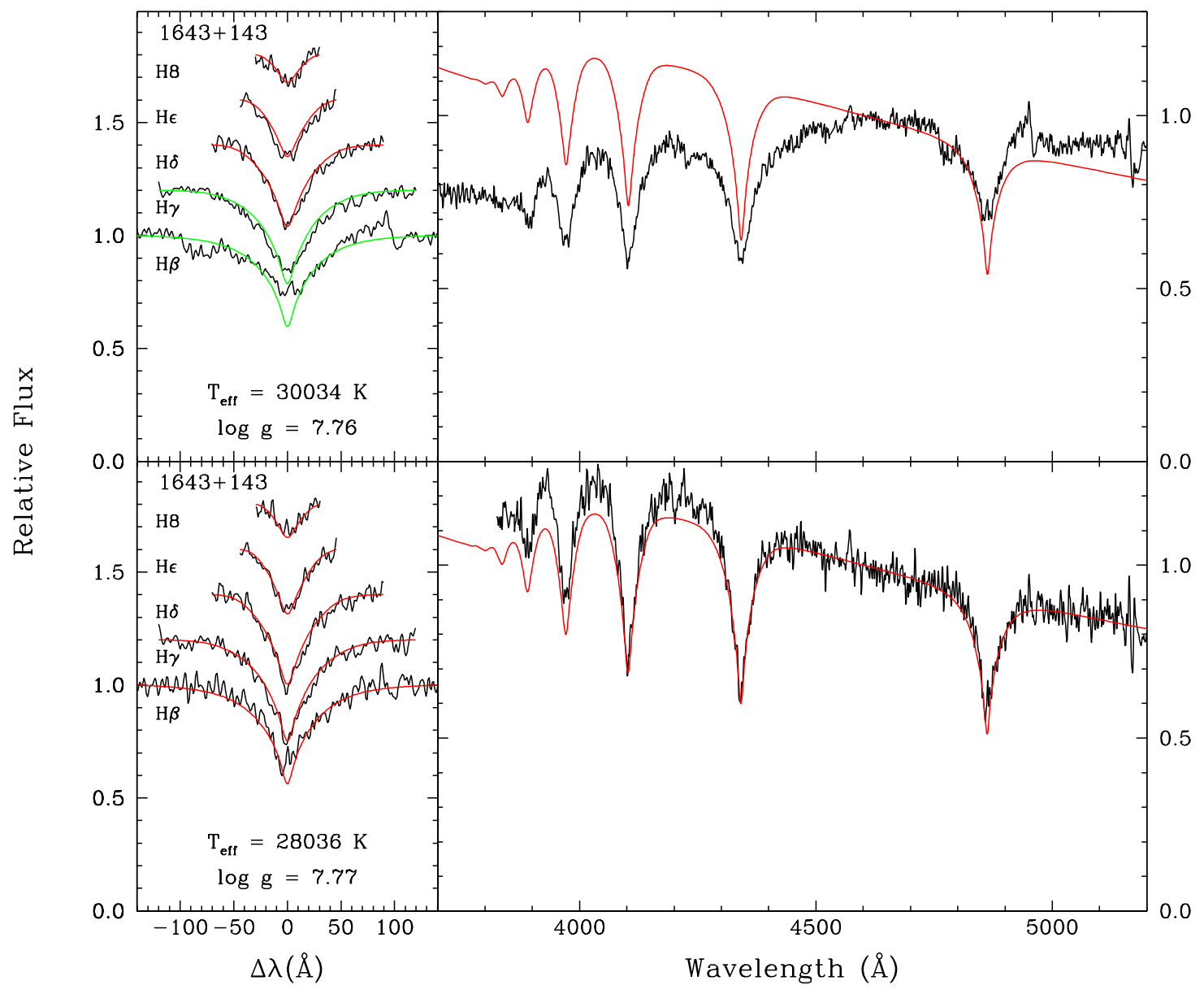

Figure 17. Comparison of the spectroscopic solutions for $1643+143$ before (top) and after (bottom) subtraction of the dM component. The left panels show the fits (red) to the observed Balmer lines (black) from $\mathrm{H} \beta$ to $\mathrm{H} 8$ where fits in green indicate spectral lines that are not taken into account in the fitting procedure. The right panels compare the observed spectrum (black) with a synthetic spectrum (red) interpolated at the values of $T_{\text {eff }}$ and $\log g$ obtained from the spectroscopic fit, both are normalized to unity at $4600 \AA$. Note the scales for the relative flux are different in the left and right panels.

synthetic spectra computed from our pure hydrogen model atmospheres with the $\mathrm{M}$ dwarf spectral templates compiled and presented by Bochanski et al. (2007). We attempt to fit a function of the form

$$
\begin{aligned}
F_{\mathrm{obs}}= & {\left[F_{\mathrm{DA}}\left(T_{\mathrm{eff}}, \log g\right)+a_{1} \cdot F_{\mathrm{dM}}(\text { Sp. type })\right] } \\
& \times\left(a_{2}+a_{3} \lambda+a_{4} \lambda^{2}+a_{5} \lambda^{3}\right)
\end{aligned}
$$

to the observed spectrum. $F_{\mathrm{DA}}$ is the flux due to the DA white dwarf and depends on $T_{\text {eff }}$ and $\log g$ while $F_{\mathrm{dM}}$, the flux from the dM component, is a function solely of the M dwarf spectral type, from M0 to M9. The remaining free parameters are $a_{1}$, which is the relative contribution of the synthetic DA spectrum and the M dwarf spectral template, $a_{2}$, a scaling factor between the composite synthetic spectrum and the observed spectrum, and the coefficients $a_{3-5}$ of a third-order polynomial that is meant to account for errors in the flux calibration. In total, there are eight free parameters when we fit the composite spectra. This means that the values of $T_{\text {eff }}, \log g$, and spectral type that we obtain are meaningless since there are too many parameters used in the fitting process. However, as we mentioned above, our only interest is to obtain the necessary function to subtract from the composite spectrum in order to remove the dM contamination. We show in Figure 16 four examples of the fits we obtain to our composite $\mathrm{DA}+\mathrm{dM}$ spectra. We see that we are able to achieve some very good fits to the observed spectra. It is interesting to note the varying degrees of contamination from one system to the next. For example, $1443+336$ shows only light contamination in contrast to $1132-298$ where the white dwarf is nearly drowned out by the flux from its $\mathrm{M}$ dwarf companion. Indeed, in the case of $1132-298$, and several other systems, when letting all the free parameters listed above vary, the fitting procedure produced rather poor results. In cases such as those, we forced $T_{\text {eff }}$ and $\log g$ to the values obtained from fitting our blue spectra of the same objects, similar to the fits presented in Figure 14, and this allowed us to achieve satisfactory results as evidenced by $1132-298$.

Once the spectroscopic fit to the composite spectrum is obtained, we subtract from the spectroscopic solution the contribution of the DA component $\left[F_{\mathrm{DA}}\left(T_{\text {eff }}, \log g\right)\right]$. In other words, we subtract a synthetic DA spectrum interpolated to the values of $T_{\text {eff }}$, and $\log g$, as determined 


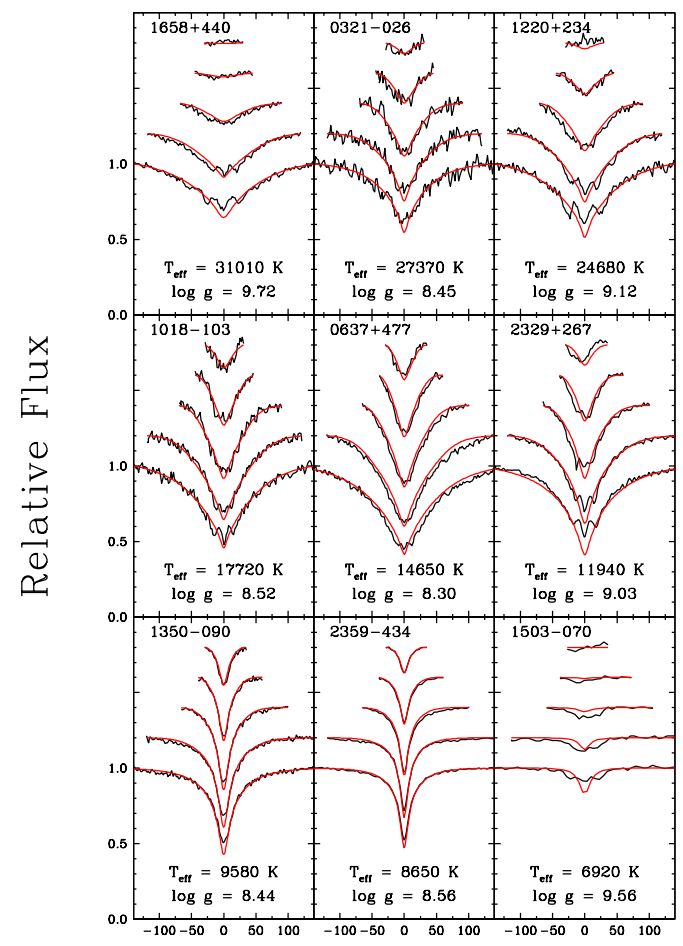

$\Delta \lambda(\AA)$

Figure 18. Same as Figure 8 for nine weakly magnetic DA white dwarfs.

by the fit to the composite spectrum, from the composite spectroscopic solution. The residual spectrum generated by this procedure represents the contribution of the $\mathrm{M}$ dwarf only. This residual spectrum is then interpolated onto the wavelength grid of the blue spectrum of the same object. Finally, we subtract the residual spectrum from the blue spectrum removing the contamination from the companion.

The next step is to fit the corrected blue spectrum with our standard fitting technique. We show in Figure 17 an example of the fits for $1643+143$ before and after the correction has been applied. We see quite a dramatic change here. Indeed, where we had to omit the fits to $\mathrm{H} \beta$ and $\mathrm{H} \gamma$, we are now able to fit them quite well with the contamination removed. The $\log g$ value is virtually identical whereas we determine a $T_{\text {eff }}$ that is over $2000 \mathrm{~K}$ lower. Finally, we note the greatly improved agreement between the slope of the observed spectrum and the spectroscopic solution.

\subsection{Magnetic and Cool White Dwarfs}

In Figure 5, we presented the spectra of 25 magnetic, or suspected to be magnetic, white dwarfs that are found in our sample. For most of these white dwarfs, our standard spectroscopic technique is inadequate since the Balmer line profiles are severely distorted and/or completely destroyed by a strong magnetic field. However, some of the more weakly magnetic stars can be fit with our spectroscopic method. We note that we do not exclude any part of the observed line profiles from our fitting procedure. Consequently, the values of $T_{\text {eff }}$ and $\log g$ derived from these fits can only be considered, at best, as
Table 3

Parameters for Magnetic White Dwarfs

\begin{tabular}{llcrcc}
\hline \hline WD & \multicolumn{1}{c}{ Name } & $\begin{array}{c}B_{p} \\
(\mathrm{MG})\end{array}$ & \multicolumn{1}{c}{$\begin{array}{c}T_{\text {eff }} \\
(\mathrm{K})\end{array}$} & $\log g$ & Notes \\
\hline $0253+508$ & KPD 0253+5052 & 17 & 15,000 & 8.00 & 1 \\
$0329+005$ & KUV 03292+0035 & 12.1 & 26,500 & $\ldots$ & 2 \\
$0553+053$ & LTT 17891 & 20 & 5500 & 8.00 & 3 \\
$0816+376$ & GD 90 & 9 & 14,000 & 8.00 & 4,5 \\
$0945+245$ & LB 11146a & 670 & 14,500 & 8.50 & $6,7,8$ \\
$1017+366$ & Ton 1206 & 65 & 16,000 & $\ldots$ & 9 \\
$1031+234$ & Ton 527 & 1000 & 15,000 & 8.50 & 10 \\
$1440+753$ & RE J1440+750 & 15 & 42,000 & 8.80 & 6,11 \\
$1506+399$ & CBS 229 & 20 & 17,000 & $\ldots$ & $6,12,13$ \\
$1533-057$ & PG 1533-057 & 31 & 20,000 & 8.50 & 1,14 \\
$1639+537$ & GD 356 & 13 & 7510 & 8.14 & 15,16 \\
$1900+705$ & LFT 1446 & 320 & 16,000 & 8.58 & 15 \\
$2316+123$ & KUV 23162+1220 & 45 & 11,000 & 8.00 & 3 \\
\hline
\end{tabular}

References. (1) Achilleos \& Wickramasinghe 1989; (2) Gänsicke et al. 2002; (3) Putney \& Jordan 1995; (4) Putnev 1997; (5) Martin \& Wickramasinghe 1984; (6) Double degenerate; (7) Liebert et al. 1993; (8) Glenn et al. 1994; (9) Saffer et al. 1989; (10) Schmidt et al. 1986; (11) Vennes et al. 1999; (12) Gianninas et al.|2009; (13) Vanlandingham et al.|2005; (14) LBH05; (15) Bergeron et al. 2001; (16) Ferrario et al. 1997.

rough estimates of the atmospheric parameters. In Figure [18, we present the fits for nine weakly magnetic white dwarfs. For $1658+440,1220+234,1018-103,0637+477$, $2329+267$, and 1503-070, the Zeeman splitting of the Balmer lines is fairly obvious. In the cases of 1350-090 and 2359-434, the spectroscopic solution predicts the Balmer lines to be deeper than observed, this is also evidence of a weak magnetic field, which is independently confirmed through spectropolarimetric measurements (Schmidt \& Smith 1994; Aznar Cuadrado et al. 2004, respectively). Finally, the reason why we classified 0321-026 as magnetic becomes evident here as the Balmer lines are again predicted to be too strong. We note that all nine stars have $\log g$ values which are substantially higher than the canonical value of $\log g=8.0$, this is not in the least surprising since it is known that magnetic white dwarfs have masses which are higher than the average for normal DA stars (Wickramasinghe \& Ferrario 2000). When trying to fit these magnetic stars with non-magnetic models, this phenomenon is exacerbated by the Zeeman splitting which broadens the lines even further causing our spectroscopic solutions to require even higher $\log g$ values. There are no better examples of this effect than $1658+440$ and $1503-070$ whose $\log g$ values have actually been extrapolated outside of our model grid.

The 16 remaining magnetic white dwarfs are simply beyond the scope of this work. The same can be said of the cool white dwarfs shown in the right panel of Figure 6. For this reason, these white dwarfs will not be further analyzed here. However, in the interest of completeness, we have searched throughout the literature in order to compile atmospheric parameter determinations, and other derived quantities, for these white dwarfs. First, Table 3 lists the properties for 13 of the 16 remaining magnetic white dwarfs, including the magnetic field strength (assuming a magnetic dipole), $T_{\text {eff }}$, and $\log g$, 
Table 4

Parameters for Cool White Dwarfs

\begin{tabular}{llccccc}
\hline \hline WD & Name & $\begin{array}{c}T_{\text {eff }} \\
(\mathrm{K})\end{array}$ & $\log g$ & $M / M_{\odot}$ & $M_{V}$ & Notes \\
\hline $0121+401$ & G133-8 & 5340 & 7.90 & 0.52 & 14.65 & 1 \\
$0213+427$ & G134-22 & 5600 & 8.12 & 0.66 & 14.72 & 1 \\
$0551+468$ & LP 159-32 & 5380 & 8.01 & 0.59 & 14.78 & 1 \\
$0648+368$ & GD 78 & 5700 & $\ldots$ & $\ldots$ & $\ldots$ & 2 \\
$0727+482 \mathrm{~A}$ & G107-70A & 5020 & 7.92 & 0.53 & 15.03 & 1,3 \\
$0727+482 \mathrm{~B}$ & G107-70B & 5000 & 8.12 & 0.66 & 15.33 & 1,3 \\
$1055-072$ & LFT 753 & 7420 & 8.42 & 0.85 & 13.91 & 1 \\
$1750+098$ & G140-B2B & 9527 & $\ldots$ & 1.17 & $\ldots$ & 4 \\
$1820+609$ & G227-28 & 4780 & 7.83 & 0.48 & 15.16 & 1 \\
\hline \multicolumn{7}{c}{ DAZ } \\
\hline $1729+371$ & GD 362 & 10,540 & 8.24 & $\ldots$ & $\ldots$ & 5 \\
\hline
\end{tabular}

References. (1) Bergeron et al. 2001; (2) Angel et al. 1981; (3) Double degenerate; (4) Silvestri et al. 2001; (5) Zuckerman et al. 2007.

where available, along with the references to the original analyses. No information could be found for $0350+098$, and $1610+330$ is identified as a magnetic white dwarf for the first time in this work. Finally, 0239+109 is analyzed at length in the next section. Similarly, Table 4 lists the atmospheric parameters for all the cool white dwarfs in our sample. Specifically, we indicate $T_{\text {eff }}, \log g$, mass, and the absolute visual magnitude along with the necessary references. We also include in Table 4 the parameters for GD 362 from Zuckerman et al. (2007) as we have not computed appropriate helium models, which include the necessary metals, for a proper re-analysis of this particular object.

We note that from here on, we shall not take into consideration the values compiled in the two tables described above in our analysis of the global properties of our spectroscopic sample. We wish to include only white dwarfs whose atmospheric parameters were determined directly by us, as we endeavor to maintain the homogeneity of our analysis.

\subsection{Peculiar Objects}

In the following section, we will take a closer look at three white dwarfs $(0239+109,0737-384$, and 0927-173) whose analysis demanded particular attention, in order to be certain we are obtaining the correct values for their atmospheric parameters. In all three cases, although the hot solution seemed to be the correct choice, based on our Balmer line fits, the slopes of the corresponding spectroscopic solutions proved incompatible with those of the observed spectra. The fits for the three stars in question are presented in Figure 19. We see that for all three stars, the hot solutions (top panels) seem to match the observed spectra rather well, whereas the cold solutions are completely incompatible with the data. We now take a closer look at each object in turn.

\subsection{1. $0239+109\left(G_{4}-34\right)$}

The star $0239+109$ is actually one of the 25 magnetic white dwarfs we presented in Figure 5 , however we will see that this case is not quite so simple. This star was analyzed in Bergeron et al. (1990a) and they had sim-

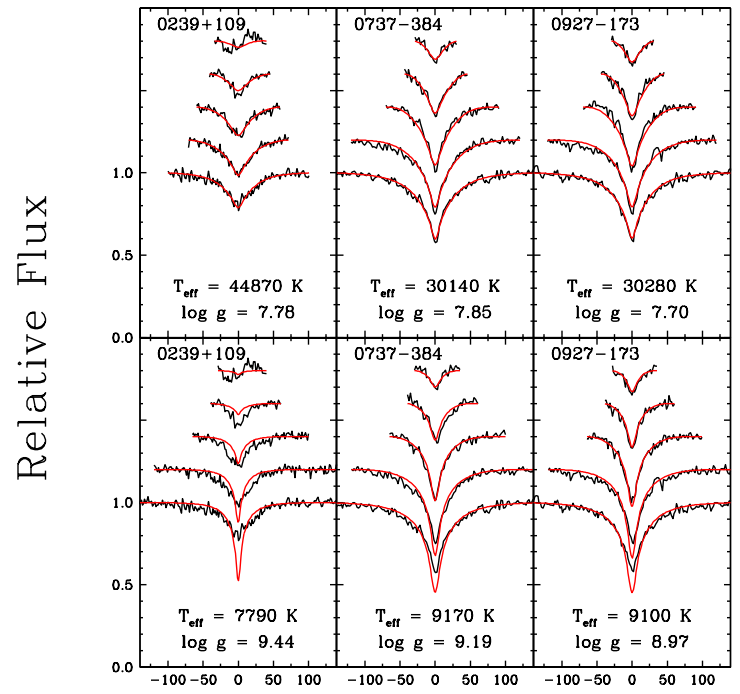

$\Delta \lambda(\AA)$

Figure 19. Model fits (red) to the observed Balmer lines (black) for the three peculiar white dwarfs in our sample using the hot seed (top) and the cold seed (bottom) for the determination of the spectroscopic solution. The atmospheric parameters for each solution are given in the figure. Both the observed and theoretical spectra are normalized to a continuum set to unity and the lines range from $\mathrm{H} \beta$ (bottom) to $\mathrm{H} 8$ (top), each offset by a factor of 0.2 .

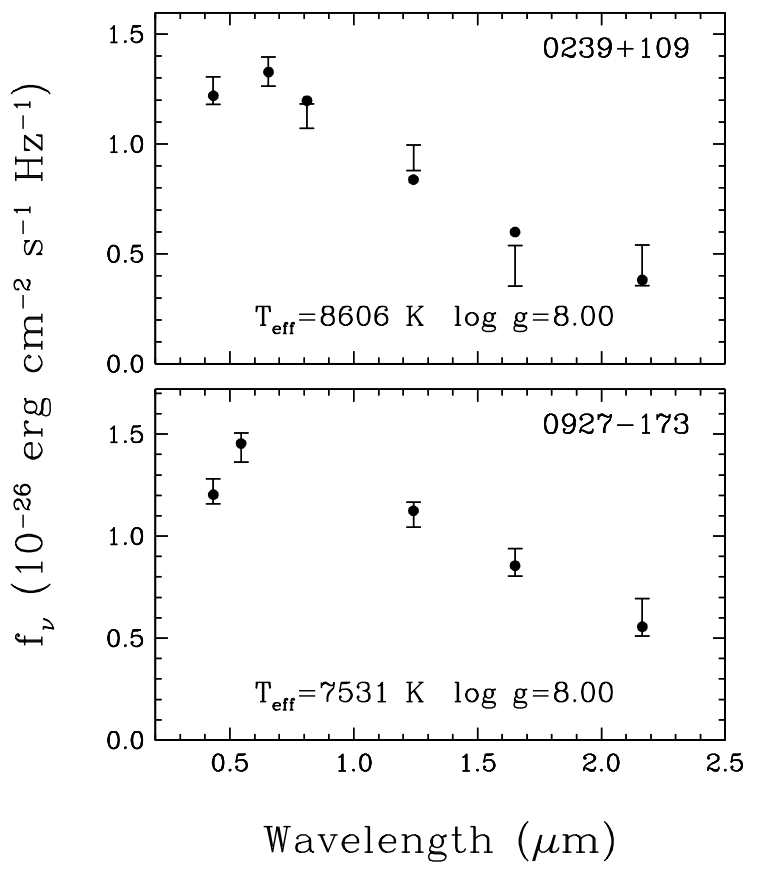

Figure 20. Fits to the energy distribution of $0239+109$ and 0927-173 with pure hydrogen models. The $B V I$ ( $B V$ only for 0927-173) and $J H K_{S}$ photometric observations are represented by error bars, while the model fluxes are shown as filled circles. The atmospheric parameters corresponding to the photometric solution are given in the figure. 
ilar difficulties in properly fitting the Balmer-line profiles (see their Figure 1). To be more exact, the slope of their observed spectrum clearly suggested a cooler spectroscopic solution despite the fact that the hot solution produced the better fit to the Balmer-line profiles. As a result, their conclusion was that $0239+109$ must in fact be an unresolved DA+DC binary system. More recently, this star was observed as part of the SPY survey and Koester et al. (2009) classified it instead as a magnetic white dwarf. Indeed, the SPY spectrum shown in Figure 7.3 of Voss (2006) shows what seems to be Zeeman splitting of both $\mathrm{H} \alpha$ and $\mathrm{H} \beta$. Our spectrum of $0239+109$ is one of the many provided to us by C. Moran, and as a result, we have no information regarding the observations (airmass, etc.). However, as we mentioned earlier, when trying to fit the Balmer-line profiles of this star, we encounter the same incoherence noted by Bergeron et al. (1990a), i.e., the hot solution produces the better fit to the observed Balmer lines, but the slope of the spectroscopic solution is incompatible with that of our observed spectrum, which suggests a much cooler temperature. Furthermore, we have in our archives the older spectrum used in the Bergeron et al. (1990a) analysis and obtained by Greenstein (1986). The slopes of the two independent observations are in perfect agreement so we are confident that there is nothing wrong with the data.

In order to get an independent estimate of $T_{\text {eff }}$, we exploit the available photometry and fit the overall optical-near-infrared spectral energy distribution combining the BRI photometry from the USNO-B Cata$\log$ (Monet et al. 2003) with the available Two Micron All Sky Survey (2MASS; Cutri et al. 2003) JHK magnitudes. Synthetic colors are obtained using the procedure outlined in Holberg \& Bergeron (2006) based on the Vega fluxes taken from Bohlin \& Gilliland (2004). The method used to fit the photometric data is described in Bergeron et al. (2001). Since we have no parallax measurement, we assume $\log g=8.0$. The best fit to the photometry is presented in the top panel of Figure 20. Although the photometric fit is not perfect, overall we see that this must be a cool object with $T_{\text {eff }} \sim 8600 \mathrm{~K}$. To reconcile the discrepancy between the slopes of the hot solution and that of our optical spectrum, taking into account the $T_{\text {eff }}$ value from the photometric fit, the only possibility is that there are two stars instead of one. Hence, we believe that in the end, both Bergeron et al. (1990a) and Koester et al. (2009) were right. In other words, the system is an unresolved doubledegenerate binary composed of a magnetic DA star and a cooler DC white dwarf, a system analogous to G62-46 (Bergeron et al. 1993). In order to get a more accurate measure of the atmospheric parameters for $0239+109$, we use a procedure identical to the one employed for the $\mathrm{DA}+\mathrm{DB}$ binary systems but here we assume a DA and a DC component. Figure 21] shows our best fit to the composite spectrum and our results are very similar to those obtained by Bergeron et al. (1990a). As a final check, we plot in Figure 22 the model flux for the combined DA+DC spectroscopic solution and compare the slope with that of our optical spectrum of $0239+109$ and see that the agreement is nearly perfect. We note, however, that despite the excellent agreement between our solution and the data, the magnetic nature of the DA component means that our measured values of $T_{\text {eff }}$ and

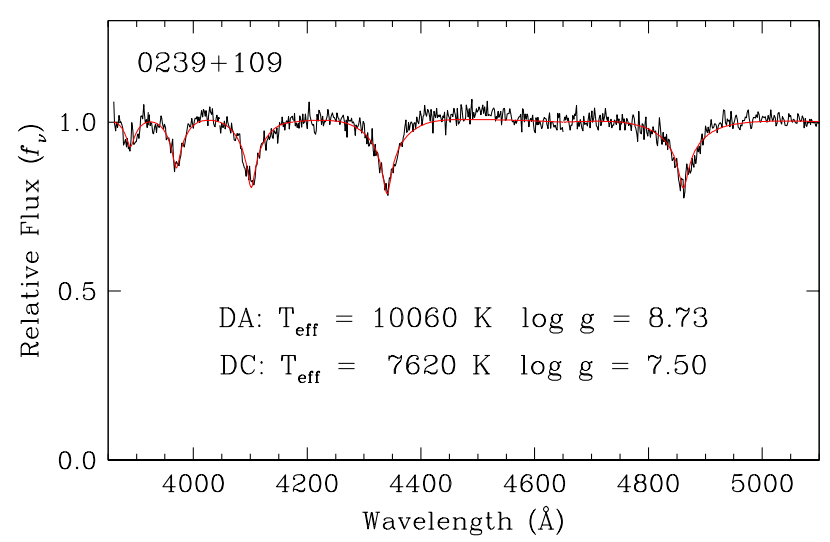

Figure 21. Model fit (red) to the optical spectrum (black) for the DA+DC binary $0239+109$. The atmospheric parameters corresponding to the spectroscopic solution are given in the figure. Both the observed and theoretical spectra are normalized to a continuum set to unity.

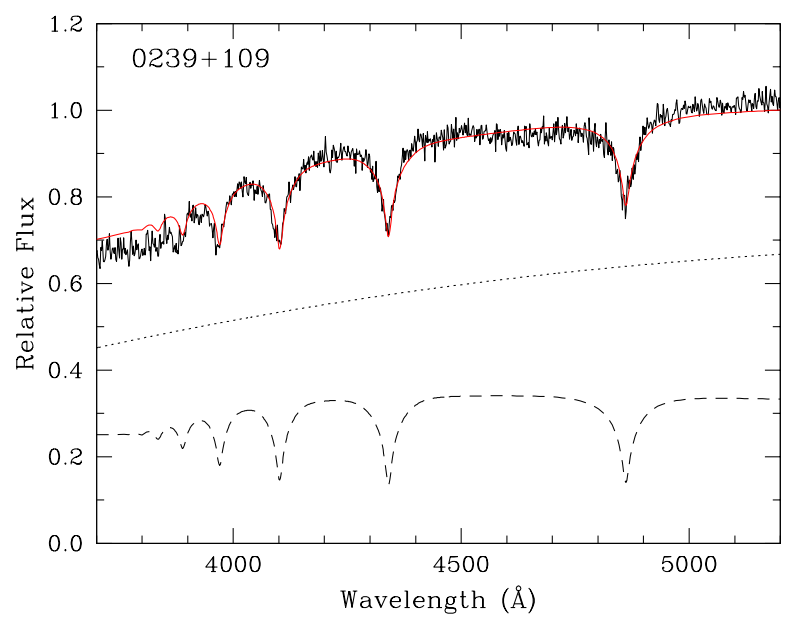

Figure 22. Relative energy distributions for our best composite $\mathrm{DA}+\mathrm{DC}$ fit displayed in Figure 21 The dashed line represents the contribution of the DA component while the dotted line represents the contribution of the DC component, both properly weighted by their respective radius. The red line corresponds to the total monochromatic flux of the composite system superimposed on our spectrum of $0239+109$ (black line), which has been scaled to the flux of the composite model at $4600 \AA$.

$\log g$ for this star should be considered approximate.

\subsection{2. $0737-384(N G C 2451-6)$}

This white dwarf is a supposed member of the open cluster NGC 2451 as first reported by Koester \& Reimers (1985). However, this claim was later challenged by Platais et al. (2001) based on the proper motion of the star as compared to the proper motion of the cluster. The analysis of Koester \& Reimers (1985) yielded $T_{\text {eff }}=$ $31,000 \pm 3000 \mathrm{~K}$, which would seem to concur with our determination assuming the hot solution is indeed the correct one. Unfortunately, the slope of the hot solution does not match the slope of our observed spectrum, which suggests a much cooler temperature for this star. How can this clear discrepancy between the slopes of our 


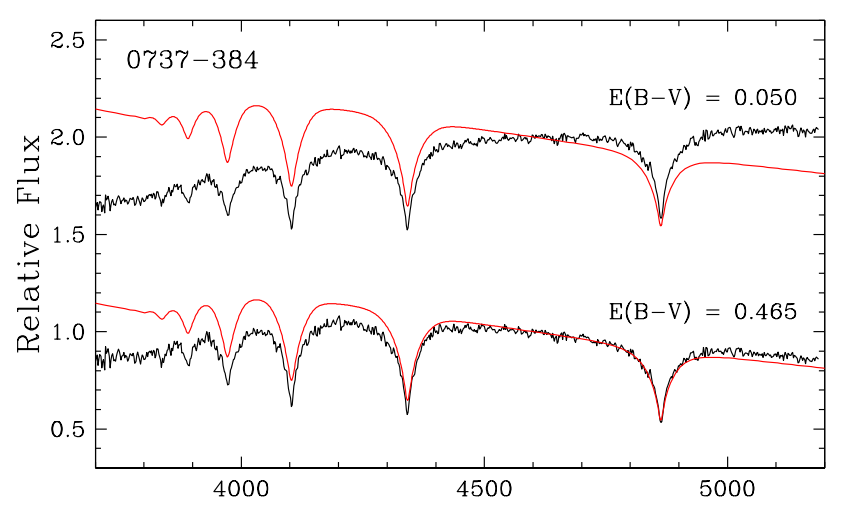

Wavelength $(\AA)$

Figure 23. Comparison of the observed spectrum (black) with the synthetic spectrum (red) corresponding to the hot solution presented in Figure 19 for 0737-384. The observed spectra have been corrected for interstellar reddening with values of $E(B-V)$ as indicated in the figure. Both the observed and synthetic spectra are normalized at $4600 \AA$ and offset vertically for clarity.

observed spectrum and that of the hot solution be explained? First, we verified our observing logs to see if our observations were obtained at high airmass. This could lead to a significant loss of flux in the blue portion of the spectrum, due to atmospheric extinction, if the instrument is not properly rotated to match the parallactic angle. As it turns out, 0737-384 was observed at an airmass of $z=1.08$ so any such effects would be minimal and we discount that possibility. Next, we attempted to fit $0737-384$ as a DA+DC system, but the slope of the combined model does not match the slope of the observed spectrum, as was the case in the previous section with $0239+109$, thus a different solution is required to explain the discrepancy. In the analysis of Koester \& Reimers (1985), a value of $E(B-V)=0.05$ is adopted for the reddening toward NGC 2451 based on several earlier studies (see references therein). Hence, we speculated that our spectrum might actually be suffering from interstellar reddening. We used the above value of interstellar reddening to correct our observed spectrum, following the prescription of Seaton (1979), but the effect is negligible as we see in the top panel of Figure 23 However, when querying the NASA/IPAC Infrared Science Archive's Dust Extinction map $\$$ in the direction of NGC 2451, we get a value of $E(B-V)=0.684$. This is significantly larger than the previously determined values. Alternatively, if we enter the coordinates for $0737-384$, we obtain $E(B-V)=0.465$. This would seem to suggest that the extinction in the direction fo the cluster is quite patchy. For our purposes, we adopt the value of $E(B-V)=0.465$. When we apply this new value of reddening to our data we see, in the bottom of Figure 23, that the slopes are now in much better agreement. We therefore conclude that the hot solution for $0737-384$ is indeed the correct one and the discrepancy between the slopes of the observed spectrum and the spectroscopic solution are due to interstellar reddening.

\footnotetext{
3 http://irsa.ipac.caltech.edu/applications/DUST/, derived using the data and techniques from [Schlegel et al. (1998).
}

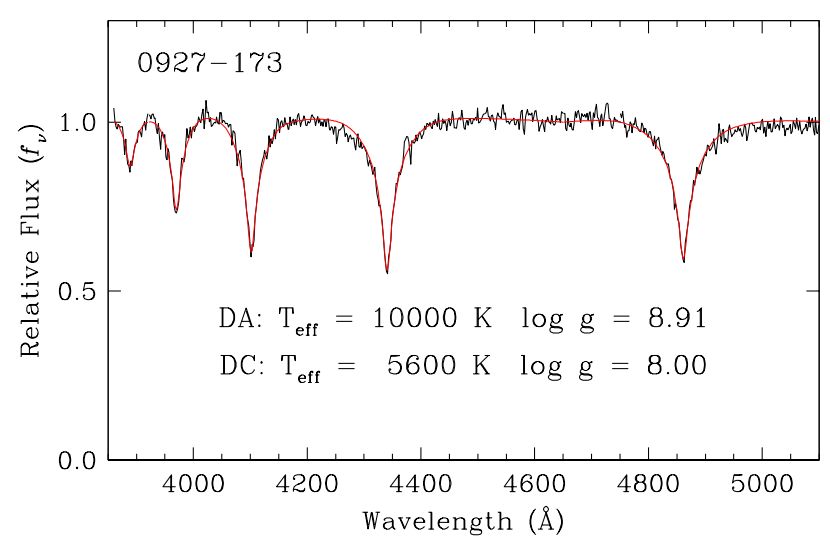

Figure 24. Model fit (red) to the optical spectrum (black) for the DA+DC binary 0927-173. The atmospheric parameters corresponding to the spectroscopic solution are given in the figure. Both the observed and theoretical spectra are normalized to a continuum set to unity.

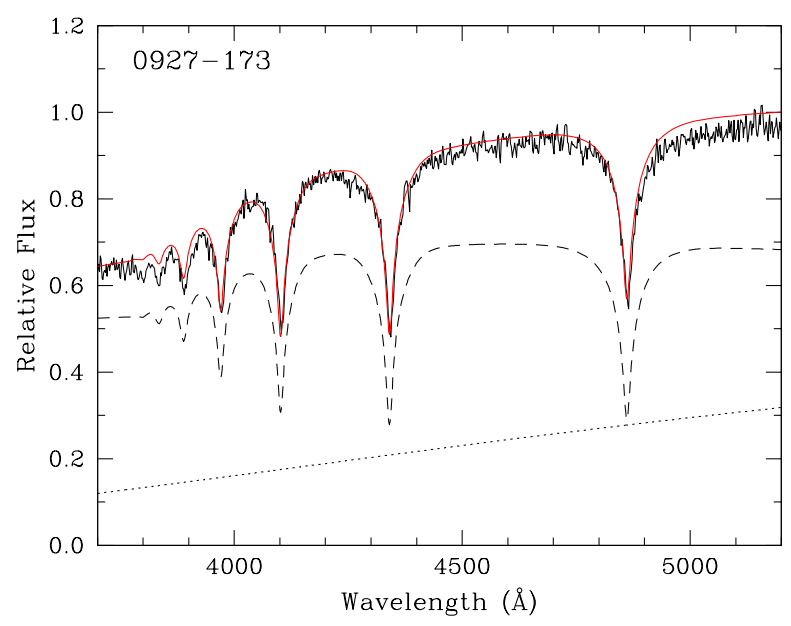

Figure 25. Relative energy distributions for our best composite DA+DC fit displayed in Figure 24 The dashed line represents the contribution of the DA component while the dotted line represents the contribution of the DC component, both properly weighted by their respective radius. The red line corresponds to the total monochromatic flux of the composite system surperimposed on our spectrum of 0927-173 (black line), which has been scaled to the flux of the composite model at $4600 \AA$.

The spectroscopic fit to the corrected spectrum yields $T_{\text {eff }}=30,270 \mathrm{~K}$ and $\log g=7.83$.

$$
\text { 4.7.3. 0927-173 (LP 787-49) }
$$

As in the previous case, our first instinct was to verify if there might be a problem with our observations of 0927-173. As it turns out, 0927-173 was observed at an airmass of $z=1.54$, which puts it at much greater risk of suffering from atmospheric extinction. However, other objects observed on the same night and at higher airmass $(z \geq 1.80)$ do not manifest the same problem and the slopes of their optical spectra are in perfect agreement with those of their spectroscopic solutions. Once again, we are confident that there is nothing wrong with the data itself. Furthermore, previous determinations of 
Table 5

Atmospheric Parameters of DA White Dwarfs from MS99

\begin{tabular}{llcccccccc}
\hline \hline WD & \multicolumn{1}{c}{ Name } & ST & \multicolumn{2}{c}{$\begin{array}{c}T_{\text {eff }} \\
(\mathrm{K})\end{array}$} & $\log g$ & $M / M_{\odot}$ & $M_{V}$ & $\begin{array}{c}\text { D } \\
(\mathrm{pc})\end{array}$ & Notes \\
\hline $0000+171$ & PG 0000+172 & DA2.4 & 21,130 & $(325)$ & $8.00(0.05)$ & $0.63(0.03)$ & 10.68 & 108 & \\
$0000-186$ & GD 575 & DA3.3 & 15,350 & $(265)$ & $7.97(0.05)$ & $0.60(0.03)$ & 11.19 & 105 & \\
$0001+433$ & RE J0003+433 & DA1.1 & $46,850(1244)$ & $9.05(0.10)$ & $1.23(0.04)$ & 11.24 & 118 & \\
$0004+061$ & PHL 670 & DA2.1 & 24,400 & $(386)$ & $8.51(0.05)$ & $0.94(0.03)$ & 11.23 & 100 & \\
$0004+330$ & GD 2 & DA1.0 & 49,980 & $(898)$ & $7.77(0.06)$ & $0.59(0.02)$ & 8.79 & 103 & \\
$0005-163$ & G158-132 & DA3.4 & 14,920 & $(252)$ & $7.93(0.05)$ & $0.57(0.03)$ & 11.18 & 104 & 1 \\
$0008+424$ & LP 192-41 & DA7.0 & 7200 & $(107)$ & $8.12(0.07)$ & $0.66(0.05)$ & 13.64 & 21 & \\
$0009+501$ & G217-37 & DA7.6 & 6620 & $(103)$ & $8.40(0.09)$ & $0.85(0.06)$ & 14.42 & 10 & 1 \\
$0009-058$ & G158-39 & DA4.8 & 10,560 & $(156)$ & $8.22(0.06)$ & $0.74(0.04)$ & 12.34 & 54 & \\
$0010+280$ & PG 0010+281 & DA1.9 & 27,220 & $(407)$ & $7.87(0.05)$ & $0.57(0.02)$ & 9.99 & 152 & \\
\hline
\end{tabular}

Notes. (1) Photometrically constant; (2) DA+dM binary; (3) ZZ Ceti; (4) Balmer line problem; (5) based on non-magnetic models; (6) DA+DC binary; (7) DA+DB binary; (8) Suffers from interstellar reddening. (Table 5 is available in its entirety in the electronic edition of the Astrophysical Journal. A portion is shown here for guidance regarding its form and content.)

$T_{\text {eff }}$ for this object suggest that it is a rather cool DA star. Indeed, Kilkennv et al. (1997) find $T_{\text {eff }}=7000 \mathrm{~K}$ while Zuckerman et al. (2003) find $T_{\text {eff }}=8000 \mathrm{~K}$, in both cases the temperature is determined solely from a measurement of $(B-V)$. Combining the photometry from Kilkenny et al. (1997) with the available measurements of $J H K_{\mathrm{s}}$ from $2 \mathrm{MASS}$, we performed a photometric fit using the same procedure described in Section 4.7.1. The results of this fit are displayed in the bottom panel of Figure 20, Assuming $\log g=8.0$, our fit to the available photometry yields $T_{\text {eff }}=7530 \mathrm{~K}$, in excellent agreement with the previous determinations, and we conclude that the cold solution must be the correct one. But how do we reconcile this temperature with the poor fit to the Balmer lines with the cold solution? The most likely explanation is that $0927-173$ is a $\mathrm{DA}+\mathrm{DC}$ binary system where the continuum flux of the DC component dilutes the observed Balmer lines leading to the results seen in Figure 19. In order to fit the composite spectrum, we employ exactly the same method as we did with the DA+DB systems analyzed in Section 4.3. Once again, $T_{\text {eff }}$ and $\log g$ for the DA component and $T_{\text {eff }}$ for the DC component are the free parameters as we fix $\log g=8.0$ for the DC star. The results of our fit are shown in Figure 24 where we see that the fit to the composite spectrum is nearly perfect. We obtain $T_{\text {eff }}=10,000 \mathrm{~K}$ and a rather high surface gravity of $\log g=8.91$ for the DA component, paired to a significantly cooler DC component with $T_{\text {eff }}=5600 \mathrm{~K}$. More importantly, in Figure 25 we compare the model flux for the combined DA+DC system to our observed spectrum and we see that the slopes are in excellent agreement. Consequently, we conclude that the observed slope of $0927-173$ can be explained by the additional presence of an unresolved companion, a cool DC white dwarf.

\section{GLOBAL PROPERTIES AND DISCUSSION}

\subsection{Adopted Atmospheric Parameters}

The atmospheric parameters we have adopted for all the stars in our sample are summarized in Table 5. This includes all the normal DA white dwarfs in our sample as well as all the stars we have analyzed in detail in the preceding sections including the DAB and DAZ stars, and the $\mathrm{DA}+\mathrm{DB}$ and $\mathrm{DA}+\mathrm{dM}$ binaries. We also include the nine weakly magnetic white dwarfs from Section 4.6 and the three peculiar objects from Section 4.7. In all, a total of 1265 white dwarfs and their atmospheric parameters are listed in Table 5. The entries in Table 5 are ordered by their WD numbers, and we list the values of $T_{\text {eff }}$ and $\log g$ as well as masses derived from the evolutionary models of Wood (1995) with thick hydrogen layers. Although the Montréal group has computed evolutionary models of their own (Fontaine et al. 2001), these are better suited for the cooler end of the white dwarf cooling sequence. At higher temperatures, the Wood and Fontaine et al. models are quite comparable and, in fact, the Wood models have the benefit of using post-asymptotic giant branch (AGB) evolutionary sequences as a starting point (M. A. Wood, 2010, private communication). Furthermore, low-mass white dwarfs, below $0.46 M_{\odot}$ and $T_{\text {eff }}<50,000 \mathrm{~K}$, are likely helium core white dwarfs, and we rely instead on the evolutionary models from Althaus et al. (2001) for those stars. For masses higher than $1.3 M_{\odot}$, we use the zero temperature calculations of Hamada \& Salpeter (1961). The values in parentheses represent the uncertainties of each parameter, calculated by combining the internal error, which is the dominant source of uncertainty, obtained from the covariance matrix of the fitting algorithm with the external error, obtained from multiple observations of the same object, estimated for DA stars at $1.2 \%$ in $T_{\text {eff }}$ and 0.038 dex in $\log g$ (see LBH05 for details). The effect on the uncertainties caused by different values of $\mathrm{S} / \mathrm{N}$ is included in the external error but is minimized by the fact that the majority of our data have rather high $\mathrm{S} / \mathrm{N}$ (see Figure 12 of Gianninas et al. 2005). In addition, we list absolute visual magnitudes determined using the photometric calibrations from Holberg \& Bergeron (2006). We also provide an updated spectroscopic classification for each star, with temperature indices based on our determinations of $T_{\text {eff }}$.

By coupling the absolute magnitudes we have deter-

4 See MS99 for a description of how the temperature index is calculated. 
mined with the apparent magnitudes available for each star in the literature, we computed spectroscopic distances that we also list in Table 5 However, these determinations come with the caveat that many of these apparent magnitudes are photographic or, when $V$ was not available, we have substituted a $B$ magnitude, or a $g$ magnitude from the SDSS, or even a Strömgren $y$ magnitude. Consequently, our distance estimates should be viewed with caution. Additionally, we applied an extinction correction of $A_{V}=1.4685$ when determining the distance to $0737-384$. We note that our distance of 142 pc for this star also places it outside NGC 2451 ( 180 pc; Platais et al. 2001).

Since there is a growing interest in identifying white dwarfs in the local neighborhood, in particular those within 20 pc (Holberg et al. 2008), we note that there is no strong evidence for new white dwarfs in our sample within that distance, with the exception perhaps of $0213+396(16 \mathrm{pc})$. The distance to $1503-070$ of 7 pc is obviously underestimated since we fitted this star with non-magnetic models (see Figure 18); the measured trigonometric parallax actually suggests a distance of $\sim$ 25 pc. We also have several objects with distances within $20 \mathrm{pc}$ that are too cool $\left(T_{\text {eff }}<6500 \mathrm{~K}\right)$ to derive meaningful atmospheric parameters with the spectroscopic technique, in particular $\log g$. One must also remember that the spectroscopic distances of cool DA stars located in the temperature range where the high-log $g$ problem is encountered are clearly underestimated since their luminosity is also underestimated (smaller radii); this includes $0213+396$, mentioned above, at $T_{\text {eff }}=9370 \mathrm{~K}$.

Finally, special notes have been added in Table 5 to designate objects that are known ZZ Ceti pulsators as well as stars that are photometrically constant. We note that all the stars analyzed in Gianninas et al. (2010) are also listed in Table 5. This includes both the DAO and $\mathrm{DA}+\mathrm{BP}$ stars, with a note for all stars that exhibit the Balmer line problem. For the DA white dwarfs that have been determined to belong to $\mathrm{DA}+\mathrm{DB}, \mathrm{DA}+\mathrm{DC}$, and $\mathrm{DA}+\mathrm{dM}$ systems, we list only the parameters of the DA component. Finally, we include a note for the weakly magnetic stars that the listed parameters were determined with non-magnetic models.

\subsubsection{Erratum: 0102-185}

One of the reported DA+BP stars from Gianninas et al. (2010), 0102-185, is actually a much cooler DA white dwarf with $T_{\text {eff }}=23,410 \mathrm{~K}$ and $\log g=7.78$, in contrast to the parameters previously reported. An error during data reduction associated the wrong spectrum to this star. The spectrum that was analyzed as 0102-185 is actually a spectrum of 2211-495 from the same observing run. This reduces the number of DA+BP stars from 18 to 17 .

\subsection{Comparison with SPY}

In establishing the reliability of the results of large surveys such as the one presented here, we believe it is both interesting and important to compare our results with other studies that include many of the same objects. As mentioned earlier, recent analyses of large num-

5 Taken from http://irsa.ipac.caltech.edu/applications/DUST/ see Section 4.7.2. bers of white dwarfs were presented both by LBH05 and Limoges \& Bergeron (2010), and although virtually all of the white dwarfs in those studies are included here, we are also using the exact same observations and analysis techniques making any meaningful comparison a rather futile endeavor. Instead, what we require is an independent analysis, which is exactly what the SPY sample analyzed by Koester et al. (2009) represents. The DA white dwarfs in the SPY sample had initially been analyzed in Voss (2006), however Koester et al. (2009) have reanalyzed the same stars with a new generation of models presented in Koester (2010). We must stress that these models did not yet include the improved Stark profiles of TB09. Consequently, as previously discussed, we will be using the results obtained with the Lemke (1997) broadening profiles in order to compare with the SPY sample. More importantly, the white dwarfs from SPY have been observed and analyzed with models and techniques completely independent from our own and thus represent a truly distinct data set.

The sample presented in Koester et al. (2009) consists of a total of 615 DA white dwarfs of which 362 are in common with our sample. The reason that there is not a greater overlap is two fold. First, many of the stars selected for the SPY project were taken from the Hamburg-ESO (Christlieb et al. 2001) and HamburgSchmidt (Homeier et al. 1998) surveys and these stars were not yet listed in MS99. Second, as we mentioned earlier, many of the white dwarfs for which we were unable to obtain spectra were situated in the southern hemisphere, and this also contributed to the smaller overlap. In order to be sure we are comparing all stars on an equal footing, we choose to compare only single DA white dwarfs; any known double-degenerate systems or magnetic white dwarfs are excluded. Furthermore, we also omit stars with $T_{\text {eff }}>50,000 \mathrm{~K}$ and $T_{\text {eff }}<8000 \mathrm{~K}$ for the same reasons outlined in Koester et al. (2009). Namely, the models used by Koester et al. (2009) do not include effects due to departures from LTE at higher temperatures, and the spectra become less sensitive to changes in $\log g$ at lower temperatures. These last two criteria reduce the number of viable white dwarfs to 330 .

The results of our comparison are presented in Figure 26. We see that with the exception of a couple of outliers near $T_{\text {eff }} \sim 14,000 \mathrm{~K}$, the agreement between our $T_{\text {eff }}$ values and those from SPY is excellent. The two outliers (0318-021 and 0937-103) are stars where both the cold and hot seeds lead to the same spectroscopic solution (see LBH05 for a discussion about the hot and cold solutions). Clearly, this is not the case in the SPY analysis of the same objects as they derive larger values for $T_{\text {eff }}$ in both cases. In contrast, the $\log g$ determinations show a clear trend where our measurements tend toward higher values than those from SPY. Koester et al. (2009) had noted this problem (see their Figure 3) and discussed several possibilities in an attempt to understand this discrepancy. In particular, they point out that differences between the "Bergeron" and "Koester" models could be the cause. Indeed, LBH05 showed a comparison between their results and those of several other studies. Three of those analyses used Koester models and all three seemed to exhibit the same trend we see here. Another possibility is that the apparent shift in $\log g$ could be caused by differences in the fitting pro- 

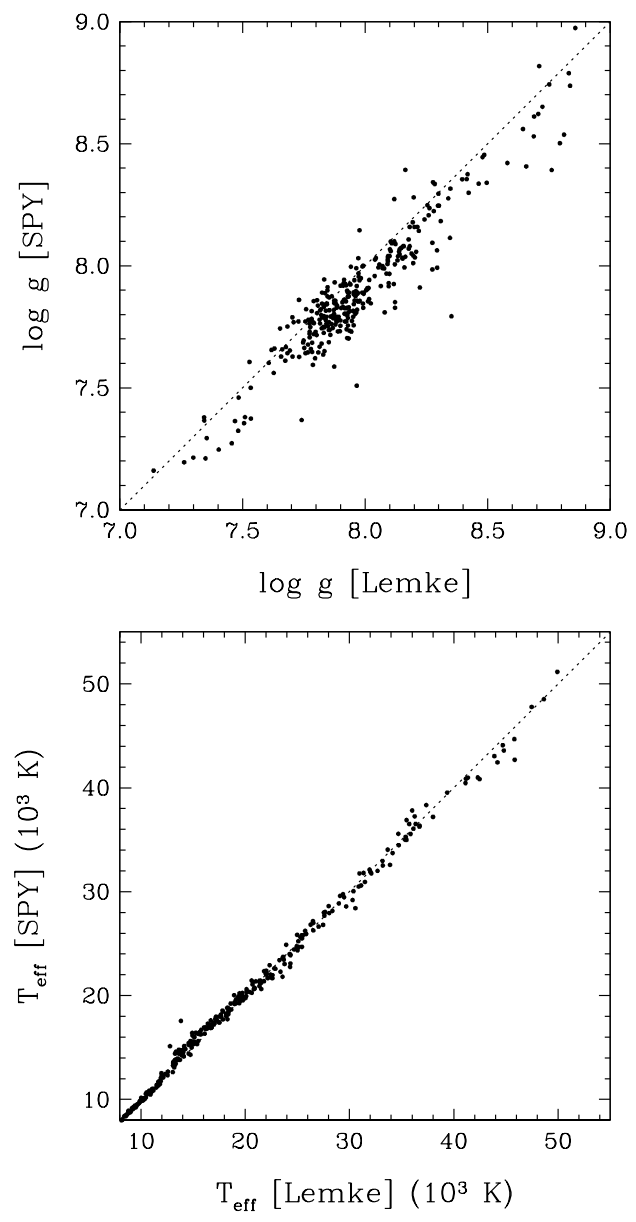

Figure 26. Comparison of $\log g$ (top) and $T_{\text {eff }}$ (bottom) values between this work using the Lemke (1997) Stark profiles and the results of Koester et al. (2009) for 330 DA white dwarfs common to both samples. The dotted lines represent the 1:1 correlation.

cedures. Finally, Koester et al. (2009) speculate that the very nature of the SPY data could be the origin of the problem. The SPY spectra were obtained with the UV-Visual Echelle Spectrograph at the Very Large Telescope, and the stitching together of the various orders might have introduced artifacts in the spectra. For the complete discussion, we refer the reader to Koester et al. (2009).

In an effort to better understand this shift in $\log g$ values, we decided to independently fit the SPY spectra, which were kindly provided to us by D. Koester, using our models and fitting procedures. In Figure 27] we show the comparison between these new values derived from the SPY spectra and those from our spectra, all analyzed with the Lemke (1997) Stark profiles. First, we note that the agreement between the $T_{\text {eff }}$ determinations (bottom panel) remains very good. Second, and more importantly, we do not observe any systematic shift in the $\log g$ values (top panel). There is more scatter in the $\log g$ plot but the overall agreement is markedly better than was seen in Figure 26. Since we obtain a satisfactory agreement between the atmospheric parameters measured from both sets of spectra, we must conclude that there is no problem, a priori, with the SPY spectra themselves. Differences between our results and those of Koester et al. (2009) must necessarily arise from dif-
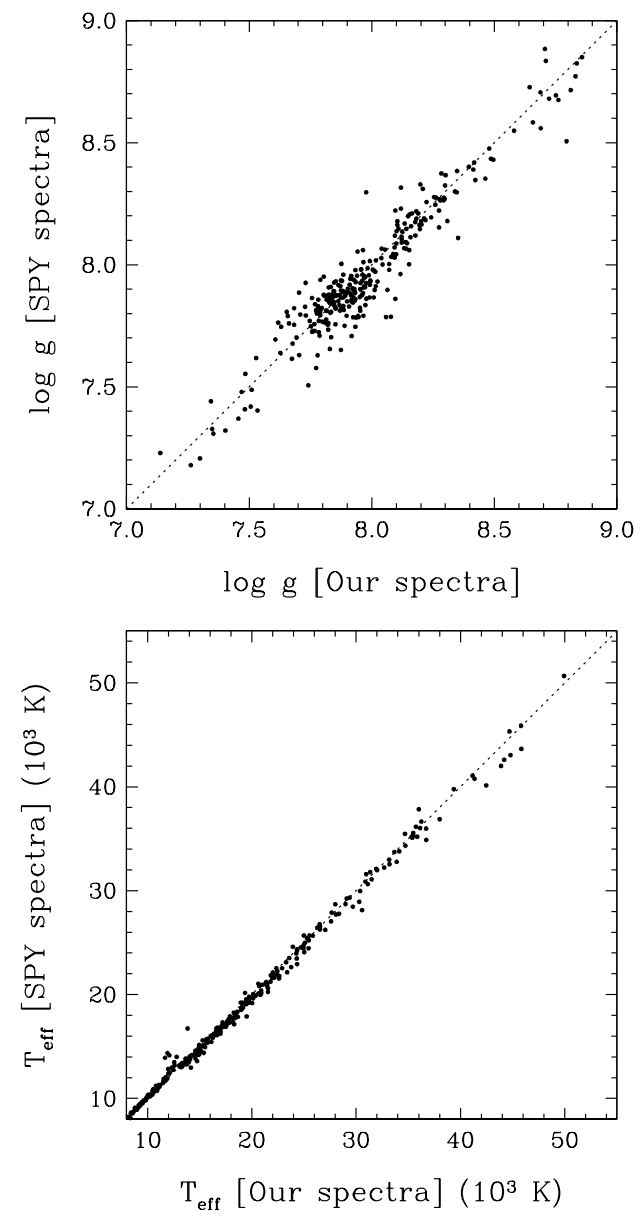

Figure 27. Comparison of $\log g$ (top) and $T_{\text {eff }}$ (bottom) values between this work using the Lemke (1997) Stark profiles and the results of our fits to the SPY spectra using the same models. The dotted lines represent the 1:1 correlation.

ferences in the models or the fitting techniques used. Nonetheless, the fact that the $T_{\text {eff }}$ determinations agree so well in both of the previous comparisons is encouraging. Furthermore, when using an independent data set and relying on our own theoretical framework, we succeed in getting both $T_{\text {eff }}$ and $\log g$ values that match rather well.

\subsection{Comparison with $S D S S$}

Another independent source of spectra is the SDSS. Despite the thousands of new white dwarfs discovered in the SDSS, very few are in common with our sample. As we showed in Figure 1, the much fainter sample of white dwarfs observed by the SDSS is the reason that the two samples are so mutually exclusive. Nonetheless, we have retained 83 DA white dwarfs in common with both samples 6 We obtained the SDSS spectra for these stars from the SDSS SkyServer 7 and proceeded to fit them with the new models that include the TB09 Stark broadening profiles. The comparison between our $T_{\text {eff }}$ and $\log g$ determinations and those obtained from the SDSS spectra is presented in Figure 28. As in the case of the comparison with SPY, we note a very good agreement between

\footnotetext{
${ }^{6}$ We omit DA+dM binaries, DAO stars, and magnetic white dwarfs from this comparison.

7 http://cas.sdss.org/dr7/en/
} 

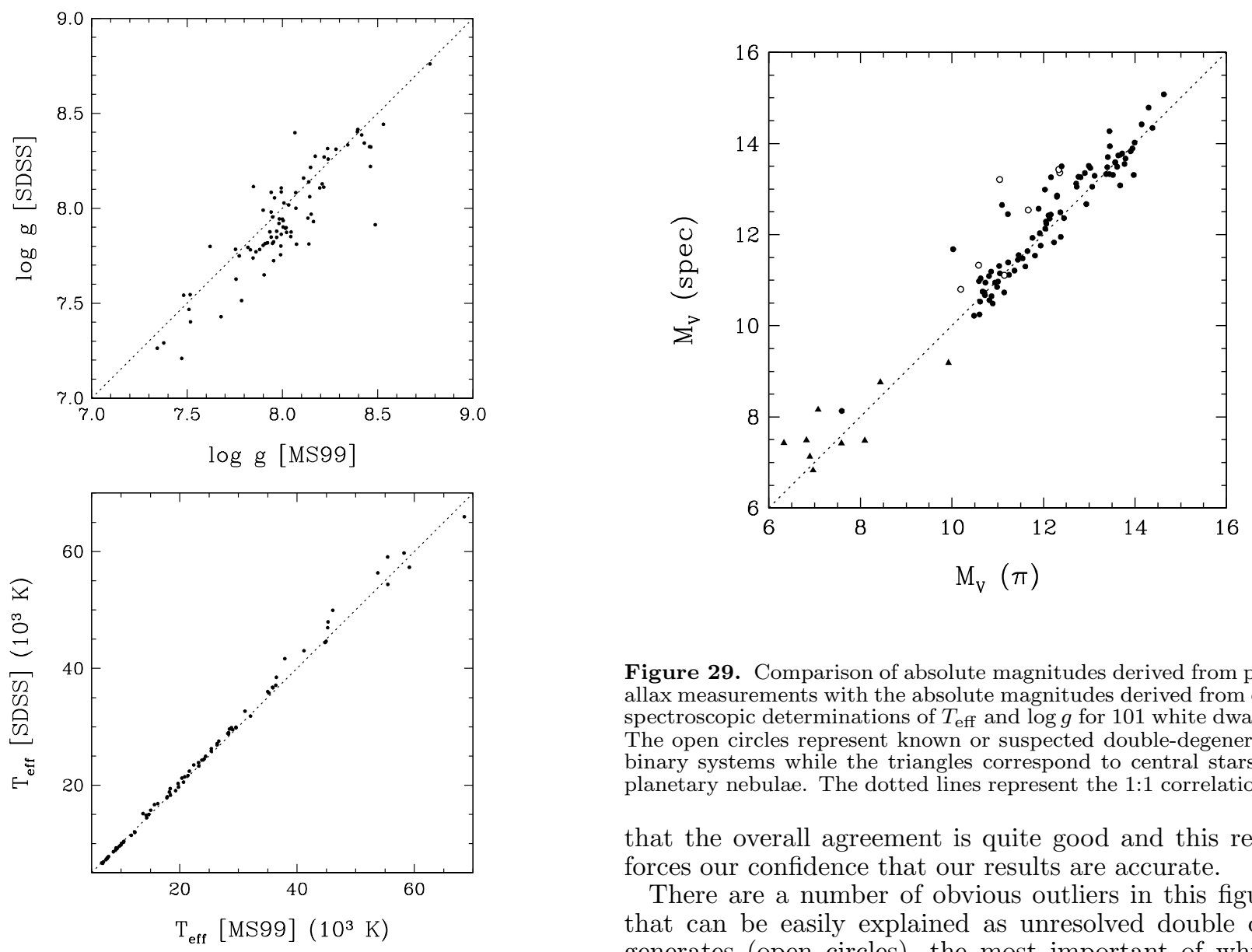

Figure 28. Comparison of $\log g$ (top) and $T_{\text {eff }}$ (bottom) values derived from our observations and from fits to SDSS spectra of 83 DA white dwarfs common to both samples. The dotted lines represent the 1:1 correlation.

both sets of $T_{\text {eff }}$ values (bottom panel). However, we also note a second similarity with our SPY comparison as the $\log g$ values also seem to be systematically shifted toward lower values from those determined from the SDSS spectra. This concurs with the results of Tremblay et al. (2011a) who explained that any differences must be due to the different spectra employed as they are being analyzed within the same theoretical framework and the same fitting method. Tremblay et al. (2011a) conclude that issues with the reduction of the SDSS data still remain and are the reason for the observed shift.

\subsection{Comparison with Parallax Measurements}

Yet another way of testing the reliability of our results is to compare our derived values of absolute magnitudes with those obtained from parallax measurements. The added incentive here is that the absolute magnitudes obtained from parallaxes are completely model independent. We have $92 \mathrm{DA}$ white dwarfs in our sample with available parallax measurements from either the Yale Parallax Catalogue (van Altena et al. 1995), or Hippar$\cos$ (Perrvman \& ESA 1997) and 9 central stars of planetary nebulae with parallaxes taken from Harris et al. (2007). We present in Figure 29 the comparison between the $M_{V}$ values from parallax measurements and those obtained through our spectroscopic analysis. We can see

Figure 29. Comparison of absolute magnitudes derived from parallax measurements with the absolute magnitudes derived from our spectroscopic determinations of $T_{\text {eff }}$ and $\log g$ for 101 white dwarfs. The open circles represent known or suspected double-degenerate binary systems while the triangles correspond to central stars of planetary nebulae. The dotted lines represent the 1:1 correlation.

that the overall agreement is quite good and this reinforces our confidence that our results are accurate.

There are a number of obvious outliers in this figure that can be easily explained as unresolved double degenerates (open circles), the most important of which is L870-2 (0135-052), discovered by Saffer et al. (1988). But this is not always the case. For instance, we find $M_{V}$ (spec) $=11.68$ for Ross $548(0133-016$, ZZ Ceti itself), for a corresponding distance of $31 \mathrm{pc}$, while the distance obtained from the trigonometric parallax measurement from the Yale Parallax Catalogue is more than twice as large at $67 \mathrm{pc}$. We cannot find an easy explanation for this discrepancy unless Ross 548 is also an unresolved degenerate binary. As well, the absolute magnitudes of cool DA stars found in the temperature range where the high- $\log g$ problem is found are likely to be overestimated; this accounts for the clump of objects away from the 1:1 correlation in the range $12.5<M_{V}<13.5$.

We must finally mention that the observed agreement is very good within the parallax uncertainties for both model grids. As TB09 pointed out, despite the fact that the new models yield higher values of $T_{\text {eff }}$ and $\log g$ (i.e., smaller radii), the two effects nearly cancel each other out and the predicted luminosities (or $M_{V}$ ) remain largely unchanged.

\subsection{Mass Distribution}

We display in Figure 30, the mass distribution as a function of $T_{\text {eff }}$ for all the stars listed in Table 5 . As previously noted, the bulk of the distribution is centered around $0.6 M_{\odot}$ for $T_{\text {eff }}>13,000 \mathrm{~K}$. Below this temperature, the well-known "high-log $g$ problem" manifests itself. Several solutions to this problem have been explored over the years. First, Bergeron et al. (1990b) showed 


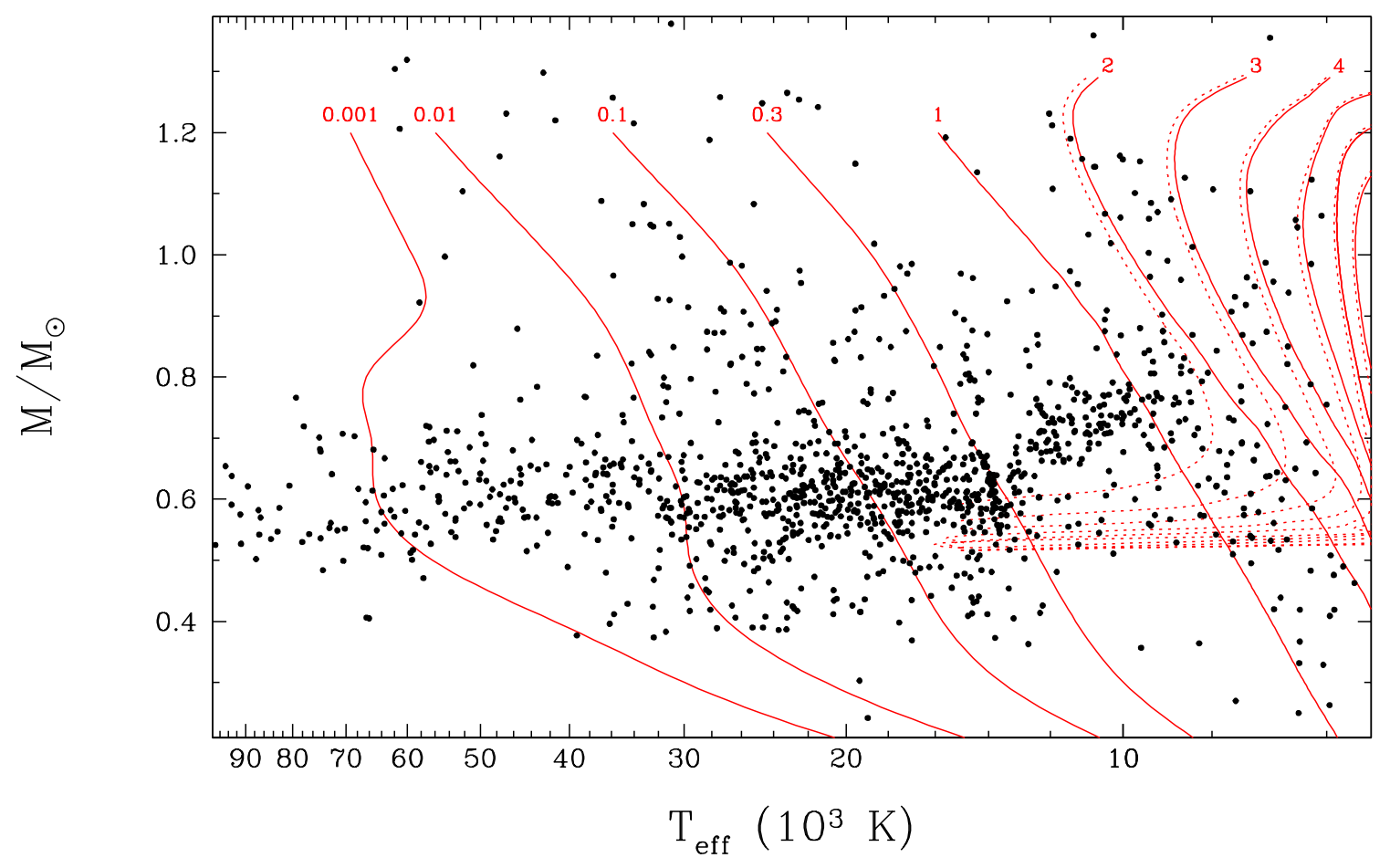

Figure 30. Mass distribution as a function of $T_{\text {eff }}$ for all the stars listed in Table 5 The solid red lines represent isochrones that take into account only the white dwarf cooling time whereas the dotted red lines include also the main-sequence lifetime. Each isochrone is labeled by its age in Gyr. Isochrones for $\tau<1$ Gyr are taken from Wood (1995) whereas those for $\tau \geq 1$ Gyr are taken from Fontaine et al. (2001).

how helium, spectroscopically invisible below 13,000 K, might explain this problem. If analyzed with pure hydrogen models, DA white dwarfs containing helium would appear to have higher $\log g$ values because the presence of helium increases the pressure in the atmosphere, which is analogous to increasing the surface gravity. Recently, Tremblay et al. (2010) attempted to detect the presence of the He I $\lambda 5877$ line in six DA white dwarfs using high-resolution spectroscopy. They found no evidence of the helium line and concluded that helium was not the solution. The reason helium was thought to be the key ingredient in explaining the high-log $g$ problem is because it was expected that convective mixing would bring it to the surface. In addition, properly calibrating the mixing-length theory used to describe convection was deemed crucial. Bergeron et al. (1995) addressed this issue but even with the mixing length properly calibrated, the high-log $g$ problem remained. The advent of the TB09 profiles was also thought to represent a possible element of the solution. However, as we have seen, the new profiles succeed in "straightening out" the mass distribution, but do not seem to contribute in fixing the masses at lower $T_{\text {eff }}$. One of the few remaining avenues of research is the computation of full three-dimensional hydrodynamic models of convection as a replacement to the mixing-length theory that has always been used, but ultimately constitutes an approximation. The preliminary results from such computations, as presented by Tremblay et al. (2011b), show that these models produce corrections to the values of $\log g$ that are of the correct magnitude and direction when compared to shifts deduced from the analysis of large samples of DA white dwarfs. Hence, it seems that the solution to the high$\log g$ problem has finally been nailed down.

At the hotter end of the white dwarf sequence, we note in Figure 30 that the distribution remains essentially continuous around $0.6 M_{\odot}$ unlike the previous determination shown in Figure 3 of Gianninas et al. (2009) where there is a noticeable "dip" toward lower masses at higher $T_{\text {eff. }}$. Our new result is a consequence of the new determinations of $T_{\text {eff }}$ and $\log g$ for the DAO stars from Gianninas et al. (2010). As it was demonstrated, the use of the CNO models to solve the Balmer line problem also resulted in higher masses for the DAO stars, consistent with the remainder of the hydrogen-rich white dwarf population.

We show in Figure 31 the histogram that represents the mass distribution of our sample regardless of effective temperature. Besides the full distribution, we also plot the mass distributions for stars above and below $T_{\text {eff }}=$ $13,000 \mathrm{~K}$ (in blue and red, respectively). The reason for this split is that we want to analyze the mass distribution for stars that are not affected by the high-log $g$ problem. The overall shape of the distribution is in good agreement with the latest determination of the SDSS DA mass distribution presented by Tremblay et al. (2011a), the only other large scale analysis of DA white dwarfs employing the new TB09 Stark profiles. In both cases, the mass distribution is strongly peaked near $0.6 M_{\odot}$. The other notable features are the high-mass tail and the low-mass component that peaks near $0.45 M_{\odot}$. The mean mass of the entire sample is $\langle M\rangle=0.661 M_{\odot}$. However, if we consider only stars with $T_{\text {eff }} \geq 13,000 \mathrm{~K}$, we get a lower mean mass of $\langle M\rangle=0.638 M_{\odot}$. This is higher 


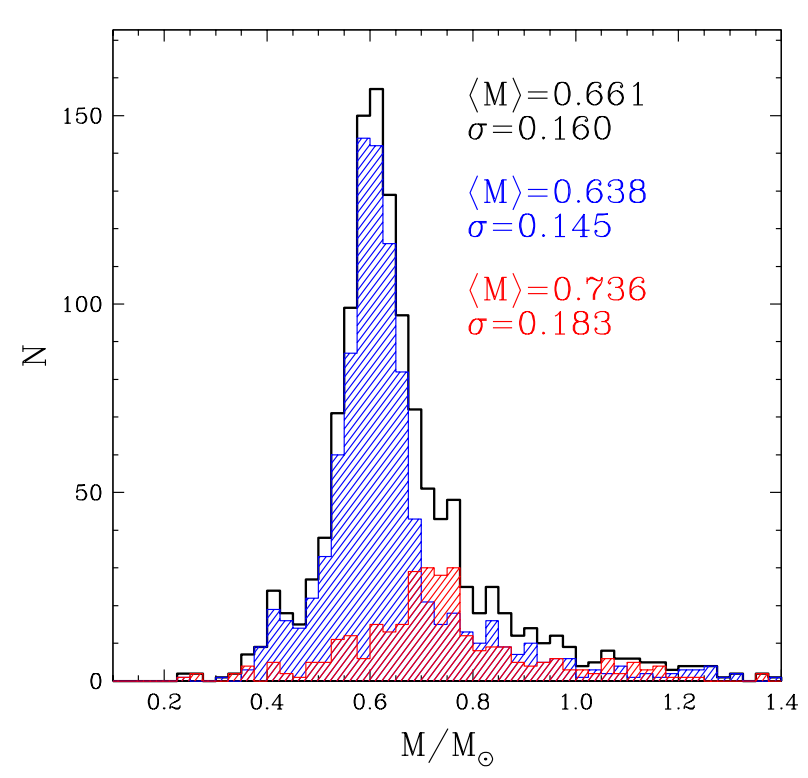

Figure 31. Mass distribution for all the stars listed in Table 5 (solid line histogram). Also shown are the mass distributions for stars with $T_{\text {eff }}>13,000 \mathrm{~K}$ (hatched blue histogram) and for stars with $T_{\text {eff }}<13,000 \mathrm{~K}$ (hatched red histogram). The mean mass and dispersion for each distribution are indicated in the figure in units of solar masses.

than the value of $0.613 M_{\odot}$ obtained by Tremblav et al. (2011a) for the SDSS DR4 sample. However, as stated in Tremblay et al., the mean mass is sensitive to outliers and as we will discuss below, the MS99 sample contains a number of high-mass white dwarfs that are systematically missed by many surveys. This fact is also evidenced in Figure 18 of Tremblay et al. (2011a) where we note a dearth of stars for $M>1.0 M_{\odot}$ and $T_{\text {eff }} \geq 20,000 \mathrm{~K}$.

We note in Figure 30 a number of rather high mass white dwarfs. In particular, if we restrict ourselves to stars with $T_{\text {eff }}>13,000 \mathrm{~K}$, thus avoiding the troublesome lower temperature regime where the high-log $g$ problem reigns, we find that there are 20 white dwarfs with a mass $M \geq 1.1 M_{\odot}$. Of these, 11 were detected by $R O S A T$ (Fleming et al. 1996). This trend is not at all surprising. By definition, white dwarfs with a higher mass have a smaller radius, which, in turn, means they are intrinsically fainter at a given $T_{\text {eff }}$. As such, magnitudelimited UV-excess surveys (PG, KUV), and many of the other surveys looking for bright blue objects (MontréalCambridge-Tololo, Edinburgh-Cape) would have skipped these stars because they are fainter. On the other hand, $\mathrm{X}$-ray surveys like the one conducted with ROSAT, cata$\log$ all sources, regardless of the brightness of the object. Consequently, since ours is not a magnitude-limited sample, these white dwarfs make their way into our sample by virtue of being included in MS99.

At the other end of the mass spectrum, the presence of stars below the dotted lines in Figure 30 is significant. Those isochrones include the main-sequence lifetime of the white dwarf's progenitor. This represents a cutoff of sorts in that the possible progenitor stars would not have had sufficient time to become white dwarfs within the lifetime of the Galaxy, nor to have cooled down to those temperatures. However, it is clear that there exists

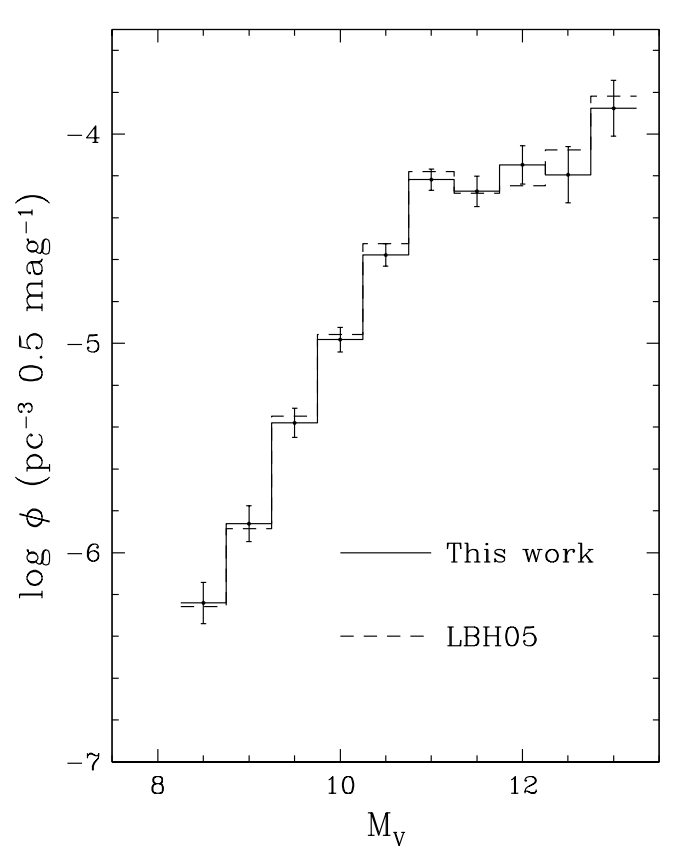

Figure 32. Luminosity function of all DA stars in the complete PG sample using the atmospheric parameters derived in this work (solid line) presented in half-magnitude bins, assuming a scale height for the Galaxy of $z_{0}=250 \mathrm{pc}$. The dashed line represents the results of LBH05 shown here for comparison.

a non-negligible low-mass component in the mass distribution. The presence of these stars can be explained if we consider binary star evolution under different guises. First, several of these stars are known to be unresolved double-degenerate binary systems that have been discovered through searches for radial velocity variations (Maxted \& Marsh 1999; Maxted et al. 2000). In such cases, the measured atmospheric parameters are misleading if we assume that only a single star is present. A second possibility involves the merger of two white dwarfs. Indeed, the recent results presented by Kilic et al. (2011, and references therein) seem to suggest that there are several very low mass $\left(\sim 0.2 M_{\odot}\right)$ white dwarfs that are in close binary systems. If these white dwarfs merge, they could potentially form a white dwarf with a mass near $0.4 M_{\odot}$. Finally, there is the possibility of a helium core white dwarf (Althaus et al. 2001). Helium core white dwarfs are formed when stars lose a significant amount of their envelope's mass to a companion before the progenitor reaches the tip of the red giant branch. Regardless which of these scenarios is the most likely to account for the population of low-mass white dwarfs, one thing is clear: these low-mass white dwarfs represent a separate evolutionary channel distinct from the usual post-AGB models that explain the evolution of the vast majority of white dwarfs.

\subsection{Revisiting the PG Luminosity Function}

Besides revising the atmospheric parameters of all the normal DA stars from the complete sample with the use of the TB09 profiles, there is also a number of white dwarfs whose atmospheric parameters are further refined from the LBH05 analysis as they are members of certain distinct classes of objects. In particular, there are 
$16 \mathrm{DA}+\mathrm{dM}$ binaries, $10 \mathrm{DAO}$ stars $8 \mathrm{DA}+\mathrm{BP}$ stars, 7 magnetic white dwarfs and finally the DA component from the $1115+166 \mathrm{DA}+\mathrm{DB}$ binary system. For the magnetic white dwarfs not included in Table [5] we adopt the parameters listed in Table 3 . With these up-to-date atmospheric parameters in hand, we proceed to recompute the PG luminosity function in exactly the same manner described in LBH05. We compare our new determination of the PG luminosity function with that of LBH05 in Figure 32. We see some variations in the individual magnitude bins with respect to the LBH05 result, but this is to be expected considering the revised atmospheric parameters we have employed in computing the luminosity function. We can also compare the space densities, in other words, the total number of DA white dwarfs per $\mathrm{pc}^{-3}$. This is determined by integrating the area under the luminosity function. LBH05 had determined a value9 of $3.07 \times 10^{-4} \mathrm{pc}^{-3}$ for $M_{V}<12.75$. Our new determination of the PG luminosity function yields a slightly lower value of $2.92 \times 10^{-4} \mathrm{pc}^{-3}$ for the same magnitude range. This represents only a $5.1 \%$ difference with the value from LBH05. At first glance, one might think that the higher temperatures produced by the new TB09 Stark profiles means that stars are intrinsically brighter than we once thought, meaning they can potentially be further away thus reducing the local density of white dwarfs. However, our earlier comparison between the absolute magnitudes obtained from spectroscopy and those determined from parallax measurements showed that the intrinsic brightness of these white dwarfs has not changed in an appreciable way since the stellar radii are also smaller (higher masses). Under these circumstances, the luminosity function should remain almost unaffected. However, closer inspection of the individual values of $T_{\text {eff }}, \log g$, and $M_{V}$ reveals that small differences do cause stars to jump from one magnitude bin to the next, which can ultimately affect the determination of the space density of white dwarfs.

\subsection{ZZ Ceti Instability Strip}

In Table 5] we list a number of stars that have been observed in high-speed photometry in order to ascertain if they are photometrically variable. In total, we identify 145 white dwarfs that are photometrically constant and 56 stars that are known ZZ Ceti pulsators. Using this photometric sample of 201 white dwarfs, we wish to revisit the empirical determination of the boundaries of the ZZ Ceti instability strip. In particular, we wish to see how the use of the TB09 Stark broadening profiles has affected the location of the instability strip as compared to our last result in Gianninas et al. (2007). We plot in Figure 33 the location of these 201 white dwarfs in the $T_{\text {eff }}-\log g$ plane. For the purposes of comparison, we plot as dotted lines the empirical boundaries of the instability strip as determined in Gianninas et al. (2007). Our new determination of these boundaries is shown as dashed lines. First, we notice that the entire strip has shifted by

8 PG 1305-017 is included in this sample but, as outlined in Gianninas et al. (2010), the parameters obtained by Bergeron et al. (1994) using their stratified models are adopted, as such, the parameters for this star remain unchanged.

${ }^{9} \mathrm{LBH} 05$ actually give a value of $5.0 \times 10^{-4} \mathrm{pc}^{-3}$ but this number is inaccurate for reasons unknown to one of the coauthors of both studies (P.B.) who obtained the correct number provided here.

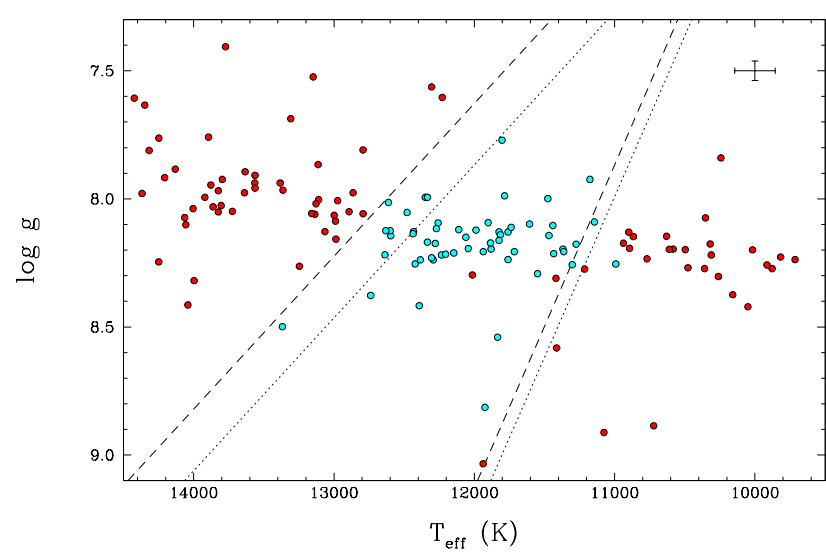

Figure 33. $T_{\text {eff }}-\log g$ distribution for DA white dwarfs with highspeed photometric measurements. The cyan circles represent the $56 \mathrm{ZZ}$ Ceti stars in our sample and the red circles are the 145 photometrically constant DA stars from Table 5. The dotted lines represent the empirical boundaries of the instability strip as established in Gianninas et al. (2006) whereas the dashed lines correspond to our new determinations. The error bars represent the average uncertainties of the spectroscopic method in the region of the ZZ Ceti instability strip.

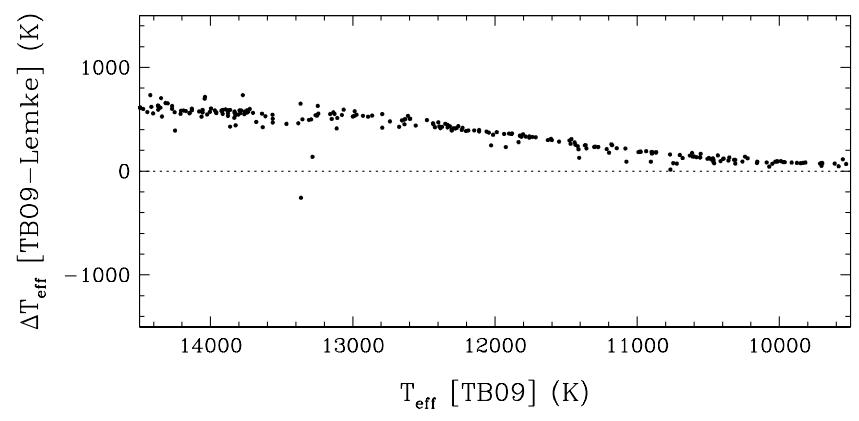

Figure 34. Differences in $T_{\text {eff }}$ determinations between the improved Stark profiles of TB09 and the profiles of Lemke (1997) as a function of $T_{\text {eff }}$ for stars near the ZZ Ceti instability strip. The dotted line represents the 1:1 correlation.

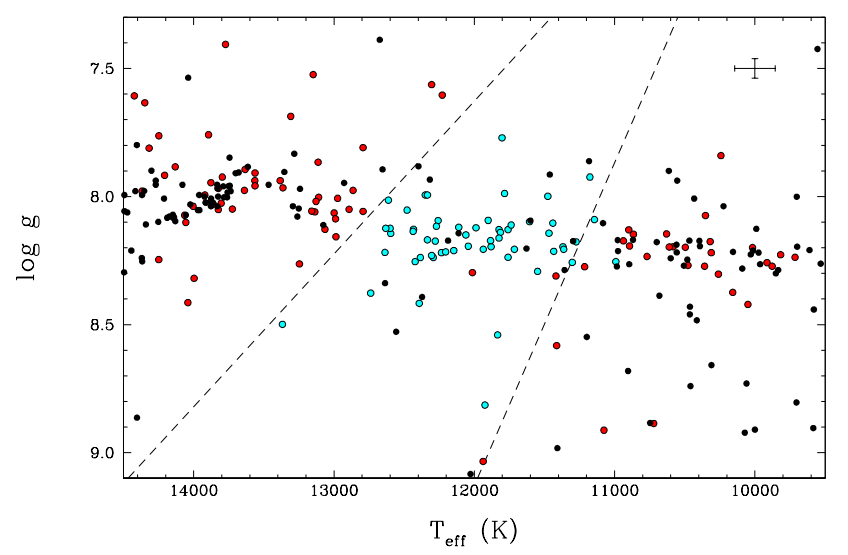

Figure 35. Same as Figure 33 but for our entire sample of white dwarfs. The black circles represent the stars for which we do not have any high-speed photometric data. The dashed lines correspond to our new determination of the empirical boundaries of the ZZ Ceti instability strip. 
a few hundred Kelvins toward higher $T_{\text {eff. }}$. Second, we see that the strip has also widened by several hundred Kelvins. These changes are a direct result of the use of the new TB09 broadening profiles. We plot in Figure 34 differences in $T_{\text {eff }}$ obtained by using the old Lemke (1997) profiles versus the calculations of TB09, focusing on the range of $T_{\text {eff }}$ where the ZZ Ceti instability strip lies. We see that the new values of $T_{\text {eff }}$ are systematically higher than the old values, this explains why the whole instability strip is shifted toward higher effective temperatures. However, we also notice that $\Delta T_{\text {eff }}$ increases as a function of $T_{\text {eff }}$ from $\sim 200 \mathrm{~K}$ near $T_{\text {eff }}=11,000 \mathrm{~K}$, to $\sim 600 \mathrm{~K}$ at $T_{\text {eff }}=13,000 \mathrm{~K}$. This is the reason that the instability strip is now wider than previously established.

One aspect of the instability strip that remains unchanged is its purity. With the exception of the two "offending" stars discussed below (one pulsator and one non-variable), only pulsating white dwarfs are to be found within the instability strip and only photometrically constant white dwarfs without, within the uncertainties. We stress that the significance of a pure instability strip is twofold. First, if the strip is pure, one can predict the variability of white dwarfs whose atmospheric parameters places them within the confines of the instability strip. Second, if the strip is pure, then it represents an evolutionary phase through which all hydrogen-rich white dwarfs must pass. As a result, asteroseismological analyses of these stars can yield important information about their internal structure, in particular the thickness of the hydrogen layer. This in turn can be applied to the entire population of hydrogen-rich white dwarf stars.

We also note that the blue edge seems to be quite clearly defined whereas there is a certain level of ambiguity associated with the location of the red edge. As far as the blue edge is concerned, the sharp transition between non-variable to variable stars is probably a consequence of very specific conditions being required for the driving mechanism to ignite pulsations at the surface of the white dwarf. On the other hand, the dying off of pulsations at the red edge is not so well understood and it is speculated that the interaction between convection and the pulsations is what ultimately signals the demise of variability. It is conceivable that this process is not as clear cut and thus the exact temperature at which a star will cease pulsating is more ambiguous, producing a red edge whose precise location is more difficult to nail down.

As mentioned above, there are two notable exceptions to the "pure" instability strip. The first of the two offending white dwarfs is $1959+059$. This is a ZZ Ceti star that lies outside the instability strip near $\sim 11,000 \mathrm{~K}$. This star was first reported as being variable by Voss et al. (2007). However, our initial spectrum for this object was one of the many provided to us by C. Moran (1999, private communication). Since the discovery of its variability is more recent, it is likely that the Moran spectrum was not averaged over several pulsation cycles as is necessary in order to obtain a proper measure of the atmospheric parameters for ZZ Ceti stars. Indeed, Voss et al. (2007) report a period of $1350 \mathrm{~s}$ and an amplitude of $5.69 \mathrm{mma}$, consistent with cooler ZZ Ceti stars that have longer periods and larger amplitudes as compared to their hotter counterparts. These large amplitudes signify that $T_{\text {eff }}$ can easily swing back and forth by several hun- dred Kelvins resulting in the star moving in and out of the instability strip (Fontaine \& Brassard 2008). However, we finally secured a new spectrum for 1959+059, averaged over several pulsation cycles, during our last observing run in 2011 April. The new values do not substantially change the position of the star in the $T_{\text {eff }}-\log g$ plane and it remains outside the empirical boundaries of the instability strip. This is not altogether inconsistent with the notion of a pure instability strip in the context of the difficulty of precisely defining the red edge of the strip, as discussed above.

The second offending star is HS $1612+5528$, the nonvariable white dwarf lying in the heart of the instability strip. This star was reported as "not observed to vary" by Voss et al. (2006). Gianninas et al. (2009) independently observed HS $1612+5528$ and also detected no variations down to a limit of $0.2 \%$. However, there are ZZ Ceti stars with amplitudes as low as $0.05 \%$. It is possible that in both cases the star was observed during a period of destructive interference. Alternatively, HS 1612+5528 could represent the first ZZ Ceti star whose pulsations are hidden from us due to geometric considerations. In other words, since we cannot resolve the surface of the star, the excited pulsation mode, or modes, might produce no detectable variations if the combined effect of the alternating hot and cold areas is nil. This would likely require a chance alignment where, for example, the rotation axis of the star is perpendicular to our line of sight. Whatever the case may be, we are planning to secure new high-speed photometry for this star during the next trimester to settle, once and for all, the issue of its photometric status.

Finally, in Figure 35 we add all the remaining stars from our survey whose photometric status is unknown. We can identify a total of 14 ZZ Ceti candidates that lie within the empirical boundaries of the instability strip. However, as Gianninas et al. (2006) demonstrated, it is important and worthwhile to observe all white dwarfs that are near either edge of the instability strip as well. It could be argued that adding a few more ZZ Ceti stars to the mix is not especially important since hundreds have now been identified through the SDSS and followup work. However, the SDSS variables are, for the most part, a few magnitudes fainter than the white dwarfs shown here. Besides the discovery light curves, it is hard to conceive of meaningful asteroseismological studies of the ZZ Ceti stars from SDSS. In contrast, these brighter candidates could be studied on small to medium-sized telescopes equipped with proper instrumentation.

The most intriguing of all the candidates is certainly $1659+662$ (GD 518). With $\log g=9.08$, it would easily become the most massive ZZ Ceti star ever discovered, surpassing even 1236-495 (BPM 37093, LTT 4816) the current heavyweight of the group. High-speed photometric measurements of this object are being planned as well.

\section{CONCLUSION}

We have presented the results of our spectroscopic survey of hydrogen-rich white dwarfs from MS99. First, we discovered that many stars listed in the catalog are still erroneously classified as white dwarfs. In particular, we list a total of 68 stars that are misclassified, 27 of which need to be reclassified as the result of our observations. 
We then examined the spectroscopic content of our survey. The majority of stars in our sample are simple DA white dwarfs but a number of them show more than just the characteristic Balmer lines of hydrogen. Indeed, our sample includes both DAB and DAZ white dwarfs as well as $\mathrm{DA}+\mathrm{dM}$ binaries and magnetic white dwarfs. In addition, there are several white dwarfs that are cool enough that no Balmer lines are present, at least not within the spectral range covered by our observations. With the exclusion of the magnetic stars and the cool, featureless white dwarfs, we have analyzed the spectra of all the white dwarfs in our sample. In order to do so, and with the goal of obtaining the most accurate atmospheric parameters possible, we have endeavored to use the most modern, up-to-date, and appropriate model atmospheres in our analyses of these white dwarfs.

First, for the analysis of all the hydrogen-rich white dwarfs we have used models that include the new Stark broadening profiles from TB09. The analysis of the hot $\mathrm{DAO}$ and $\mathrm{DA}+\mathrm{BP}$ stars was presented in Gianninas et al. (2010) and featured models to which $\mathrm{CNO}$, at solar abundances, was added in order to overcome the Balmer-line problem. The results of that analysis were incorporated here. Furthermore, we used updated helium atmosphere models to analyze several $\mathrm{DA}+\mathrm{DB}$ double-degenerate binary systems and we developed a technique that uses $\mathrm{M}$ dwarf templates to correct for the contamination in the composite spectra of $\mathrm{DA}+\mathrm{dM}$ binary systems. We also analyzed several DAZ stars by including calcium in the calculation of the synthetic spectrum only. Two of these are newly identified DAZ stars.

In switching from the older Stark broadening profiles of Lemke (1997) to those of TB09, we also explored how our atmospheric parameters have changed as a result. We observed that the values of $T_{\text {eff }}$ are shifted toward higher temperatures while the values of $\log g$ show a more obvious and significant trend toward higher values. With that said, we also saw how the new profiles improved the overall shape of the mass distribution as a function of $T_{\text {eff }}$. The distribution now remains largely constant around $0.60 M_{\odot}$ until $\sim 13,000 \mathrm{~K}$ where the well-known high-log $g$ problem takes over. The new three-dimensional hydrodynamic models of convection presented in Tremblay et al. (2011b) have finally, and convincingly, solved this problem by replacing the more approximate mixing-length theory used to this day.

We also wanted to test the reliability of our results by comparing them with those of an independent study. The Koester et al. (2009) analysis of DA white dwarfs from the SPY sample was ideal for this purpose since several hundred white dwarfs were in common with both samples but were observed and analyzed in a completely independent fashion. We showed that our $T_{\text {eff }}$ determinations are in excellent agreement with those of Koester et al. (2009) despite a trend toward higher $\log g$ values in the Koester et al. analysis.

Our mass distribution yields a mean mass of $0.638 M_{\odot}$ when considering only white dwarfs with $T_{\text {eff }} \geq 13,000 \mathrm{~K}$. The mean mass is somewhat higher than other determinations, but it is understood to be caused by high-mass white dwarfs that are missed in many surveys but included here. We also note the presence of a low-mass component, consistent with the results of most other large-scale studies of DA white dwarfs. The low-mass stars are necessarily the product of binary evolution either through mergers or a common envelope phase where a significant fraction of their mass may have been lost.

We also recomputed the PG luminosity function and found a space density which is only slightly lower than that determined by LBH05. It would be easy to point the finger at the new TB09 Stark broadening profiles since they yield higher temperatures, but they also yield higher $\log g$ measurements that translate to smaller radii. These facts coupled together produce two very similar values of $M_{V}$. However, even small differences can cause stars to switch magnitude bins, which explains our marginally lower value.

Finally, we re-examined the ZZ Ceti instability strip and found that the TB09 Stark broadening profiles produce a wider instability strip that is also systematically shifted toward higher $T_{\text {eff }}$. Furthermore, we have identified over a dozen new ZZ Ceti candidates among which might be the most massive ZZ Ceti star ever discovered. High-speed photometric observations are planned for all candidates in the very near future.

We thank the director and staff of Steward Observatory and of the Carnegie Observatories for the use of their facilities. We are also grateful to D. Koester for providing us with all of the SPY spectra, M.-M. Limoges for obtaining several of the optical spectra analyzed in this paper, and R. Heller for inadvertant help with Table 5. This work was supported in part by the NSERC Canada and by the Fund FQRNT (Québec). M.T.R. acknowledges support from FONDAP (15010003) and Proyecto BASAL PB06 (CATA). P.B. is a Cottrell Scholar of Research Corporation for Science Advancement.

\section{REFERENCES}

Achilleos, N., \& Wickramasinghe, D. T. 1989, ApJ, 346, 444

Althaus, L. G., Serenelli, A. M., \& Benvenuto, O. G. 2001, MNRAS, 323, 471

Angel, J. R. P., Borra, E. F., \& Landstreet, J. D. 1981, ApJS, 45, 457

Asplund, M., Grevesse, N., \& Sauval, A. J. 2005, in ASP Conf. Ser. 336, Cosmic Abundances as Records of Stellar Evolution and Nucleosynthesis, ed. T. G. Barnes, III \& F. N. Bash (San Frnacisco, CA: ASP), 25

Aznar Cuadrado, R., Jordan, S., Napiwotzki, R., et al. 2004, A\&A, 423, 1081

Beauchamp, A. 1995, PhD thesis, Univ. de Montréal

Beauchamp, A., Wesemael, F., \& Bergeron, P. 1997, ApJS, 108, 559

Beauchamp, A., Wesemael, F., Bergeron, P., Liebert, J., \& Saffer, R. A. 1996, in ASP Conf. Ser. 96, Hydrogen-Deficient Stars, ed. S. Jeffery \& U. Heber (San Francisco, CA: ASP), 295

Beauchamp, A., Wesemael, F., Bergeron, P., et al. 1999, ApJ, 516,887

Becklin, E. E., Farihi, J., Jura, et al. 2005, ApJ, 632, L119

Bergeron, P., Greenstein, J. L., \& Liebert, J. 1990a, ApJ, 361, 190

Bergeron, P., Leggett, S. K., \& Ruiz, M. T. 2001, ApJS, 133, 413

Bergeron, P., \& Liebert, J. 2002, ApJ, 566, 1091

Bergeron, P., Ruiz, M. T., \& Leggett, S. K. 1993, ApJ, 407, 733

Bergeron, P., Saffer, R. A., \& Liebert, J. 1992, ApJ, 394, 228

Bergeron, P., Wesemael, F., Beauchamp, A., Wood, M. A.,

Lamontagne, R., Fontaine, G., \& Liebert, J. 1994, ApJ, 432, 305

Bergeron, P., Wesemael, F., Dufour, P., et al. 2011, ApJ, 432, 305

Bergeron, P., Wesemael, F., Fontaine, G., \& Liebert, J. 1990b, ApJ, 351, L21

Bergeron, P., Wesemael, F., Lamontagne, et al. 1995, ApJ, 449, 258 
Billères, M., Wesemael, F., Bergeron, P., \& Beauchamp, A. 1997, ApJ, 488, 368

Bochanski, J. J., West, A. A., Hawley, S. L., \& Covey, K. R. 2007, AJ, 133, 531

Bohlin, R. C., \& Gilliland, R. L. 2004, AJ, 127, 3508

Bowyer, S., Lieu, R., Lampton, M., et al. 1994, ApJS, 93, 569

Catalán, S., Isern, J., García-Berro, E., et al. 2008, A\&A, 477, 213

Christlieb, N., Wisotzki, L., Reimers, D., et al. 2001, A\&A, 366, 898

Cutri, R. M., Skrutskie, M. F., van Dyk, S., et al. 2003, The IRSA 2MASS All-Sky Point Source Catalog, NASA/IPAC Infrared Science Archive, http://irsa.ipac.caltech.edu

Dufour, P., Fontaine, G., Liebert, J., Schmidt, G. D., \& Behara, N. 2008, ApJ, 683, 978

Eisenstein, D. J., Liebert, J., Harris, H. C., et al. 2006, ApJS, 167,40

Farihi, J., Becklin, E. E., \& Zuckerman, B. 2005, ApJS, 161, 394

Ferrario, L., Wickramasinghe, D. T., Liebert, J., Schmidt, G. D., \& Bieging, J. H. 1997, MNRAS, 289, 105

Finley, D. S., Koester, D., \& Basri, G. 1997, ApJ, 488, 375

Fleming, T. A., Snowden, S. L., Pfeffermann, E., Briel, U., \& Greiner, J. 1996, A\&A, 316, 147

Fontaine, G., \& Brassard, P. 2008, PASP, 120, 1043

Fontaine, G., Brassard, P., \& Bergeron, P. 2001, PASP, 113, 409

Gänsicke, B. T., Euchner, F., \& Jordan, S. 2002, A\&A, 394, 957

Gänsicke, B. T., Koester, D., Girven, J., Marsh, T. R., \& Steeghs, D. 2010, Science, 327, 188

Gänsicke, B. T., Koester, D., Marsh, T. R., Rebassa-Mansergas, A., \& Southworth, J. 2008, MNRAS, 391, L103

Gianninas, A., Bergeron, P., Dupuis, J., \& Ruiz, M. T. 2010, ApJ, 720,581

Gianninas, A., Bergeron, P., \& Fontaine, G. 2005, ApJ, 631, 1100

Gianninas, A., Bergeron, P., \& Fontaine, G. 2006, AJ, 132, 831

Gianninas, A., Bergeron, P., \& Fontaine, G. 2007, in ASP Conf. Ser. 372, 15th European Workshop on White Dwarfs, ed. R. Napiwotzki \& M. R. Burleigh, (San Francisco, CA:ASP), 577

Gianninas, A., Bergeron, P., \& Ruiz, M. T. 2009, J. Phys.: Conf. Ser., 172, 012021

Gianninas, A., Dufour, P. \& Bergeron, P. 2004, ApJ, 617, L57

Giclas, H. L., Burnham, R., \& Thomas, N. G. 1965, Lowell Obs. Bull., 6, 155

Glenn, J., Liebert, J., \& Schmidt, G. D. 1994, PASP, 106, 722

Green, R. F., Schmidt, M., \& Liebert, J. 1986, ApJS, 61, 305

Greenstein, J. L. 1986, ApJ, 304, 334

Hamada, T., \& Salpeter, E. E. 1961, ApJ, 134, 683

Harris, H. C., Dahn, C. C., Canzian, B., et al. 2007, AJ, 133, 631

Heber, U., Napiwotzki, R., Lemke, M., \& Edelmann, H. 1997, A\&A, 324, L53

Heller, R., Homeier, D., Dreizler, S., \& Østensen, R. 2009, A\&A, 496, 191

Holberg, J. B., \& Bergeron, P. 2006, AJ, 132, 1221

Holberg, J. B., Sion, E. M., Oswalt, T., et al. 2008, AJ, 135, 1225

Homeier, D., Koester, D., Hagen, H.-J., et al. 1998, A\&A, 338, 563

Hubeny, I., \& Lanz, T. 1995, ApJ, 439, 875

Hügelmeyer, S. D., Dreizler, S., Homeier, et al., 2006, A\&A, 454, 617

John, T. L. 1994, MNRAS, 269, 871

Jordan, S., Heber, U., Engels, D., \& Koester, D. 1993, A\&A, 273 , L27

Kawka, A., Vennes, S., \& Thorstensen, J. R. 2004, AJ, 127, 1702

Kepler, S. O., Kleinman, S. J., Nitta, A., et al. 2007, MNRAS, 375,1315

Kilic, M., Brown, W. R., Allende Prieto, C., et al. 2011, ApJ, 727,3

Kilic, M., von Hippel, T., Leggett, S. K., \& Winget, D. E. 2005, ApJ, 632, L115

Kilkenny, D., Heber, U., \& Drilling, J. S. 1988, South Afr. Astron. Obs. Cir., 12, 1

Kilkenny, D., O'Donoghue, D., Koen, C., Stobie, R. S., \& Chen, A. 1997, MNRAS, 287,867

Koester, D. 2010, Mem. Soc. Astron. Ital., 81, 921

Koester, D., Napiwotzki, R., Christlieb, N., et al. 2001, A\&A, 378,556

Koester, D., \& Reimers, D. 1985, A\&A, 153, 260

Koester, D., Voss, B., Napiwotzki, R., et al. 2009, A\&A, 505, 441
Kondo, M., Noguchi, T., \& Maehara, H. 1984, Ann. Tokyo Astron. Obs., 20, 130

Lacombe, P., Wesemael, F., Fontaine, G., \& Liebert, J. 1983, ApJ, 272, 660

Lemke, M. 1997, A\&AS, 122, 285

Liebert, J., Bergeron, P., \& Holberg, J. B. 2005, ApJS, 156, 47 (LBH05)

Liebert, J., Bergeron, P., Schmidt, G. D., \& Saffer, R. A. 1993, ApJ, 418, 426

Limoges, M.-M., \& Bergeron, P. 2010, ApJ, 714, 1037

Lisker, T., Heber, U., Napiwotzki, R., et al. 2005, A\&A, 430, 223

Marsh, M. C., Barstow, M. A., Buckley, D. A., et al. 1997a, MNRAS, 286, 369

Marsh, M. C., Barstow, M. A., Buckely, D. A., et al. 1997b, MNRAS, 287, 705

Martin, B., \& Wickramasinghe, D. T. 1984, MNRAS, 206, 407

Maxted, P. F. L., \& Marsh, T. R. 1999, MNRAS, 307, 122

Maxted, P. F. L., Marsh, T. R., \& Moran, C. K. J. 2000,

MNRAS, 319, 305

McCook, G. P., \& Sion, E. M. 1999, ApJS, 121, 1 (MS99)

Monet, D. G., Levine, S. E., Canzian, B., et al. 2003, AJ, 125, 984

Noguchi, T., Maehara, H., \& Kondo, M. 1980, Ann. Tokyo Astron. Obs., 18, 55

Pereira, C., Bergeron, P., \& Wesemael, F. 2005, ApJ, 623, 1076

Perryman, M. A. C., \& ESA 1997, The Hipparcos and Tycho Catalogues (ESA SP-1200; Noordwijk: ESA)

Platais, I., Kozhurina-Platais, V., Barnes, S., et al. 2001, AJ, 122, 1486

Pounds, K. A., Allan, D. J., Barber, C., et al. 1993, MNRAS, 260, 77

Press, W. H., Flannery, B. P., Teukolsky, S. A., \& Vetterling, W. T. 1986, Numerical Recipes (Cambridge: Cambridge Univ. Press)

Putney, A. 1997, ApJS, 112, 527

Putney, A., \& Jordan, S. 1995, ApJ, 449, 863

Rebassa-Mansergas, A., Gänsicke, B. T., Schreiber, M. R.,

Koester, D., \& Rodríguez-Gil, P. 2010, MNRAS, 402, 620

Saffer, R. A., Liebert, J., \& Olszewski, E. M. 1988, ApJ, 334, 947

Saffer, R. A., Liebert, J., Wagner, R. M., Sion, E. M., \&

Starrfield, S. G. 1989, AJ, 98, 668

Schlegel, D. J., Finkbeiner, D. P., \& Davis, M. 1998, ApJ, 500 525

Schmidt, G. D., \& Smith, P. S. 1994, ApJ, 423, L63

Schmidt, G. D., West, S. C., Liebert, J., Green, R. F., \&

Stockman, H. S. 1986, ApJ, 309, 218

Seaton, M. J. 1979, MNRAS, 187, 73P

Silvestri, N. M., Hawley, S. L., West, A. A., et al. 2006, AJ, 131, 1674

Silvestri, N. M., Oswalt, T. D., Wood, M. A., et al. 2001, AJ, 121, 503

Smith, R. J., Croom, S. M., Boyle, B. J., et al. 2005, MNRAS, 359,57

Strand, K. A., Dahn, C. C., \& Liebert, J. W. 1976, BAAS, 8, 506

Stroeer, A., Heber, U., Lisker, T., et al. 2007, A\&A, 462, 269

Subasavage, J. P., Henry, T. J., Bergeron, P., et al. 2007, AJ, 134, 252

Tremblay, P.-E., \& Bergeron, P. 2009, ApJ, 696, 1755 (TB09)

Tremblay, P.-E., Bergeron, P., \& Gianninas, A. 2011a, ApJ, 730, 128

Tremblay, P.-E., Bergeron, P., Kalirai, J. S., \& Gianninas, A. 2010, ApJ, 712, 1345

Tremblay, P.-E., Ludwig, H.-G., Steffen, M., Bergeron, P., \& Freytag, B. 2011b, A\&A, 531, L19

van Altena, W. F., Lee, J. T., \& Hoffleit, E. D. 1995, The General Catalogue of Trigonometric Parallaxes (New Haven, CT: Yale Univ. Obs.)

Vanlandingham, K. M., Schmidt, G. D., Eisenstein, D. J., et al. 2005, AJ, 130, 734

Vennes, S., Christian, D. J., \& Thorstensen, J. R. 1998, ApJ, 502, 763

Vennes, S., Ferrario, L., \& Wickramasinghe, D. T. 1999, MNRAS, 302, L49

Vennes, S., Smith, R. J., Boyle, B. J., et al. 2002, MNRAS, 335, 673

Vennes, S., Thejll, P. A., Galvan, R. G., \& Dupuis, J. 1997, ApJ, 480, 714 
Vennes, S., Thejll, P. A., Wickramasinghe, D. T., \& Bessell, M. S. 1996, ApJ, 467, 782

Voss, B. 2006, PhD thesis, Univ. Kiel

Voss, B., Koester, D., Østensen, R., et al. 2006, A\&A, 450, 1061

Voss, B., Koester, D., Østensen, R., et al. 2007, in ASP Conf. Ser. 372,15 th European Workshop on White Dwarfs, ed. R.

Napiwotzki \& M. R. Burleigh, (San Francisco, CA: ASP), 583

Werner, K. 1996, ApJ, 457, L39

Wesemael, F., Bergeron, P., Lamontagne, R. L., et al. 1994, ApJ, 429,369
Wickramasinghe, D. T., \& Ferrario, L. 2000, PASP, 112, 873

Wolff, B., Jordan, S., Koester, D., \& Reimers, D. 2000, A\&A, 361,629

Wood, M. A. 1995, in Proceedings of the 9th European Workshop on White Dwarfs, ed. D. Koester \& K. Werner (Berlin:

Springer), 41

Zuckerman, B., Koester, D., Melis, C., Hansen, B. M., \& Jura, M. 2007, ApJ, 671, 872

Zuckerman, B., Koester, D., Reid, I. N., \& Hünsch, M. 2003, ApJ, 596,477 